\title{
Tropospheric Measurements of \\ Gas-Phase and Aerosol Chemistry \\ in Polar Regions
}

\section{Compiled by Rolf Sander}

I received tremendous help and many suggestions for additional references from Jan Bottenheim. In addition, for pointing out miscellaneous data sets I would like to thank Parisa Ariya, Harry Beine, Torunn Berg, Gao Chen, Jack Dibb, Ralf Ebinghaus, Markus Frey, Udo Friess, Dwayne Heard, Manuel Hutterli, Hans-Werner Jacobi, Lars Kaleschke, Karin Kreher, Samuel Morin, Andreas Richter, Joel Savarino, Paul Shepson, Henrik Skov, Franz Slemr, Sverre Solberg, Alexandra Steffen, Bill Sturges, Roland von Glasow, Yuhang Wang, and Eric Wolff.
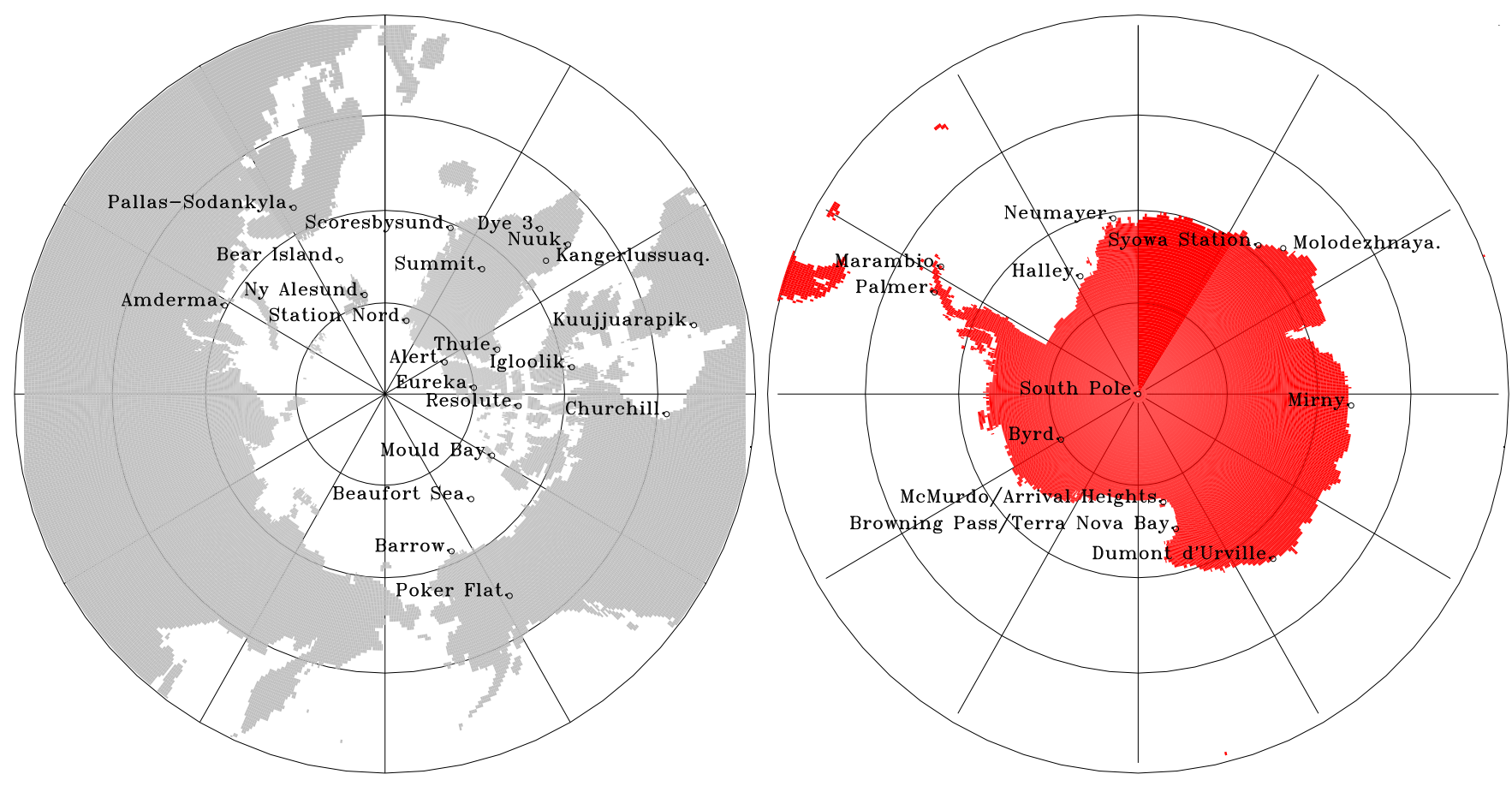

Version from January 23, 2007

This compilation is published as an electronic supplement to the AICI review paper: "Halogens and their role in polar boundary-layer ozone depletion" by Simpson et al., Atmos. Chem. Phys., 2007, available at: http://www . atmos-chem-phys .org. 


\section{Introduction}

Many measurements of tropospheric gas-phase and aerosol chemistry in polar regions have been made and subsequently published in miscellaneous journals. In this compilation, an attempt is made to provide a concise overview of these measurements. The tables do not claim completeness but it is hoped they they can serve as a starting point when searching for data of a particular compound. $\mathrm{CO}_{2}$ is not included because gathering all data for this species would be beyond the scope of this work.

There are two main tables: The first contains measurements of gas-phase species, and the second contains aerosol measurements. The measurement sites are listed in Tab. 1, and also shown on the map on the title page.

In some cases, concentrations in $\left[\mathrm{ng} / \mathrm{m}^{3}\right]$ from the original papers were converted to mixing ratios assuming a molar volume of $20 \mathrm{l} / \mathrm{mol}$ for the cold air.

Several abbreviations and acronyms are used in the tables, as explained below:

\begin{tabular}{|c|c|c|c|}
\hline date: & $\begin{array}{l}\text { spr } \\
\text { sum } \\
\text { fal } \\
\text { win }\end{array}$ & $\begin{array}{l}= \\
= \\
= \\
=\end{array}$ & $\begin{array}{l}\text { spring } \\
\text { summer } \\
\text { fall } \\
\text { winter }\end{array}$ \\
\hline \multirow[t]{3}{*}{ mercury: } & GEM & $=$ & gaseous elementary mercury \\
\hline & TGM & $=$ & total gaseous mercury \\
\hline & RGM & $=$ & reactive gaseous mercury \\
\hline \multirow[t]{3}{*}{ other: } & bgr & $=$ & background air \\
\hline & $\mathrm{DL}$ & $=$ & detection limit \\
\hline & SCD & $=$ & slant column density \\
\hline
\end{tabular}

In addition to the data presented in the peer-reviewed literature, there are several web sites providing large data sets.

- http://gaw.kishou.go.jp/wdcgg.html

The World Data Centre for Greenhouse Gases (WDCGG) is established under the Global Atmosphere Watch (GAW) programme to collect, archive and provide data for greenhouse $\left(\mathrm{CO}_{2}, \mathrm{CH}_{4}, \mathrm{CFCs}, \mathrm{N}_{2} \mathrm{O}\right.$, etc.) and related $\left(\mathrm{CO}, \mathrm{NO}_{\mathrm{x}}, \mathrm{SO}_{2}, \mathrm{VOC}\right.$, etc.) gases and surface ozone in the atmosphere and ocean, measured under GAW and other programmes. From this web site, you can obtain information including WDCGG's publications and measurement data that have been contributed by organizations and individual researchers in the world.

- http://www.cmdl.noaa.gov/ccgg/iadv/

The Interactive Atmospheric Data Visualization web page provides data for $\mathrm{CH}_{4}, \mathrm{CO}, \mathrm{CO}_{2}, \mathrm{H}_{2}, \mathrm{~N}_{2} \mathrm{O}, \mathrm{SF}_{6}$, and other gases. The global coverage includes several Arctic and Antarctic stations.

- http://www. amap.no/Assessment/ScientificBackground.htm

The Arctic Monitoring and Assessment Programme (AMAP) offers several scientific reports.

- http://www.awi-bremerhaven.de/GPH/AirChemistryObservatory/scientific_program.html A summary of the actual sampling program realised at the Air Chemistry Observatory of the Neumayer Station. 
Table 1: List of measurement Sites

\begin{tabular}{|c|c|c|c|c|}
\hline abbrev & name & longitude & latitude & altitude $^{a}$ \\
\hline $\mathrm{AL}$ & Alert, NWT, Canada & $82^{\circ} 27^{\prime} \mathrm{N}$ & $62^{\circ} 31^{\prime} \mathrm{W}$ & $210 \mathrm{~m}$ a.s.l. \\
\hline $\mathrm{AM}$ & Amderma, Russia & $69.72^{\circ} \mathrm{N}$ & $61.62^{\circ} \mathrm{E}$ & \\
\hline ant & Antarctic (miscellaneous sites) & - & - & \\
\hline $\mathrm{ARC}$ & Arctic (miscellaneous sites) & - & - & \\
\hline $\mathrm{BA}$ & Barrow, Alaska, USA & $71^{\circ} 19^{\prime} \mathrm{N}$ & $156^{\circ} 36^{\prime} \mathrm{W}$ & $8 \mathrm{~m}$ a.s.l. \\
\hline $\mathrm{BI}$ & Bear Island & $74.5^{\circ} \mathrm{N}$ & $19.0^{\circ} \mathrm{E}$ & \\
\hline $\mathrm{bp}$ & Browning Pass, Ross Sea, Antarctica & $74^{\circ} 36^{\prime} \mathrm{S}$ & $163^{\circ} 56^{\prime} \mathrm{E}$ & \\
\hline BS & Beaufort Sea & $\approx 75^{\circ} \mathrm{N}$ & $\approx 140^{\circ} \mathrm{W}$ & \\
\hline by & Byrd Station & $80.00^{\circ} \mathrm{S}$ & $120.00^{\circ} \mathrm{W}$ & \\
\hline $\mathrm{CH}$ & Churchill, Canada & $59^{\circ} \mathrm{N}$ & $94^{\circ} \mathrm{W}$ & \\
\hline $\mathrm{du}$ & Dumont d'Urville, Antarctica & $66^{\circ} 40^{\prime} \mathrm{S}$ & $140^{\circ} 01^{\prime} \mathrm{E}$ & $40 \mathrm{~m}$ a.s.l. \\
\hline DY & Dye 3, Greenland & $65.2^{\circ} \mathrm{N}$ & $43.8^{\circ} \mathrm{W}$ & \\
\hline $\mathrm{EU}$ & Eureka, Canada & $80^{\circ} \mathrm{N}$ & $86^{\circ} \mathrm{W}$ & \\
\hline ha & Halley, Antarctic & $75^{\circ} 35^{\prime} \mathrm{S}$ & $26^{\circ} 39^{\prime} \mathrm{W}$ & $32 \mathrm{~m}$ a.s.l. \\
\hline $\mathrm{HU}$ & Hudson Bay, Canada & $\approx 55^{\circ} \mathrm{N}$ & $\approx 75^{\circ} \mathrm{W}$ & \\
\hline IG & Igloolik, NWT, Canada & $69^{\circ} \mathrm{N}$ & $82^{\circ} \mathrm{W}$ & \\
\hline KA & Kangerlussuaq (Søndre Strømfjord), Greenland & $67^{\circ} \mathrm{N}$ & $51^{\circ} \mathrm{W}$ & \\
\hline KU & Kuujjuarapik, Quebec, Canada & $55.5^{\circ} \mathrm{N}$ & $77.7^{\circ} \mathrm{W}$ & \\
\hline ma & Marambio, Antarctic & $64.2^{\circ} \mathrm{S}$ & $57.7^{\circ} \mathrm{W}$ & \\
\hline MB & Mould Bay, NWT, Canada & $76^{\circ} 15^{\prime} \mathrm{N}$ & $119^{\circ} 20^{\prime} \mathrm{W}$ & $58 \mathrm{~m}$ a.s.l. \\
\hline $\mathrm{mi}$ & Mirny, Antarctic & $66.33^{\circ} \mathrm{S}$ & $93.01^{\circ} \mathrm{E}$ & \\
\hline $\mathrm{mm}$ & McMurdo station, Arrival Heights, Antarctic & $77^{\circ} 49^{\prime} \mathrm{S}$ & $166^{\circ} 35^{\prime} \mathrm{E}$ & $11 \mathrm{~m}$ a.s.l. \\
\hline mo & Molodezhnaya, Antarctic & $67.4^{\circ} \mathrm{S}$ & $45.5^{\circ} \mathrm{E}$ & \\
\hline NA & Ny Ålesund Zeppelin Station, Spitzbergen, Norway & $78^{\circ} 54^{\prime} \mathrm{N}$ & $11^{\circ} 52^{\prime} \mathrm{E}$ & $475 \mathrm{~m}$ a.s.l. \\
\hline $\mathrm{nm}$ & Neumayer Station, Antarctic & $70^{\circ} 39^{\prime} \mathrm{S}$ & $8^{\circ} 15^{\prime} \mathrm{W}$ & $42 \mathrm{~m}$ a.s.l. \\
\hline $\mathrm{NO}$ & Norwegian Arctic (miscellaneous sites) & - & - & \\
\hline NU & Nuuk, Greenland & $64^{\circ} 06^{\prime} \mathrm{N}$ & $51^{\circ} 24^{\prime} \mathrm{W}$ & \\
\hline NW & Narwhal ice floe camp, Arctic (140 km NW of Alert) & $83^{\circ} 54^{\prime} \mathrm{N}$ & $63^{\circ} 17^{\prime} \mathrm{W}$ & \\
\hline pa & Palmer Station, Antarctic & $64^{\circ} 55^{\prime} \mathrm{S}$ & $64^{\circ} 00^{\prime} \mathrm{W}$ & $10 \mathrm{~m}$ a.s.l. \\
\hline $\mathrm{PF}$ & Poker Flat, Alaska, USA & $64^{\circ} 11^{\prime} \mathrm{N}$ & $147^{\circ} 43^{\prime} \mathrm{W}$ & $501 \mathrm{~m}$ a.s.l. \\
\hline PS & Pallas-Sodankylä, Finland & $67^{\circ} 22^{\prime} \mathrm{N}$ & $26^{\circ} 39^{\prime} \mathrm{E}$ & \\
\hline $\mathrm{RE}$ & Resolute, Canada & $75^{\circ} \mathrm{N}$ & $95^{\circ} \mathrm{W}$ & \\
\hline $\mathrm{SC}$ & Scoresbysund, Greenland & $70^{\circ} 29^{\prime} \mathrm{N}$ & $21^{\circ} 58^{\prime} \mathrm{W}$ & \\
\hline $\mathrm{SN}$ & Station Nord, Greenland & $81^{\circ} 36^{\prime} \mathrm{N}$ & $16^{\circ} 40^{\prime} \mathrm{W}$ & \\
\hline sp & South Pole & $90^{\circ} \mathrm{S}$ & - & $2810 \mathrm{~m}$ a.s.l. \\
\hline SU & Summit, Greenland & $72^{\circ} 35^{\prime} \mathrm{N}$ & $38^{\circ} 29^{\prime} \mathrm{W}$ & $3238 \mathrm{~m}$ a.s.l. \\
\hline SW & SWAN ice floe camp, Arctic (160 km N of Alert) & $83.9^{\circ} \mathrm{N}$ & $63.1^{\circ} \mathrm{W}$ & \\
\hline sy & Syowa Station, Antarctic & $69^{\circ} 00^{\prime} \mathrm{S}$ & $39^{\circ} 35^{\prime} \mathrm{E}$ & \\
\hline $\mathrm{TH}$ & Thule, Greenland & $76^{\circ} 31^{\prime} \mathrm{N}$ & $68^{\circ} 50^{\prime} \mathrm{W}$ & \\
\hline tn & Terra Nova Bay, Antarctic & $74^{\circ} 42^{\prime} \mathrm{S}$ & $164^{\circ} 6^{\prime} \mathrm{E}$ & \\
\hline
\end{tabular}




\begin{tabular}{|c|c|c|c|c|}
\hline species & value & date & site & reference \\
\hline \multicolumn{5}{|c|}{$* * * * *$ Gas-phase Species $* * * * *$} \\
\hline \multicolumn{5}{|c|}{ Oxygen and Hydrogen } \\
\hline $\mathrm{O}_{3}$ & & 1958 & ha & Roscoe and Roscoe (2006) \\
\hline $\mathrm{O}_{3}$ & & $1966-2000$ & $\mathrm{RE}$ & Tarasick and Bottenheim (2002) \\
\hline $\mathrm{O}_{3}$ & & 1973-1978 & $\mathrm{BA}$ & Oltmans (1981) \\
\hline $\mathrm{O}_{3}$ & & 1973-1984 & $\mathrm{BA}$ & Oltmans and Komhyr (1986) \\
\hline $\mathrm{O}_{3}$ & & 1974-2000 & $\mathrm{CH}$ & Tarasick and Bottenheim (2002) \\
\hline $\mathrm{O}_{3}$ & & $1975-1978$ & $\mathrm{sp}$ & Oltmans (1981) \\
\hline $\mathrm{O}_{3}$ & & $1975-1984$ & $\mathrm{sp}$ & Oltmans and Komhyr (1986) \\
\hline $\mathrm{O}_{3}$ & & $1975-2004$ & $\mathrm{BA}, \mathrm{sp}$ & Oltmans et al. (2006) \\
\hline $\mathrm{O}_{3}$ & $\begin{array}{l}\text { monthly mean: } \\
\mathrm{nmol} / \mathrm{mol}\end{array}$ & 1975-1989 & $\mathrm{sp}$ & Schnell et al. (1991) \\
\hline $\mathrm{O}_{3}$ & $\begin{array}{l}\text { monthly mean: } \\
\text { nmol/mol }\end{array}$ & 1975-1989 (Feb) & $\mathrm{sp}$ & Schnell et al. (1991) \\
\hline $\mathrm{O}_{3}$ & $\begin{array}{l}\text { monthly mean: } \\
\mathrm{nmol} / \mathrm{mol}\end{array}$ & 1975-1989 (Aug) & $\mathrm{sp}$ & Schnell et al. (1991) \\
\hline $\mathrm{O}_{3}$ & & 1979-1986 & $\mathrm{ARC}$ & Oltmans et al. (1989) \\
\hline $\mathrm{O}_{3}$ & $17 \ldots 37 \mathrm{nmol} / \mathrm{mol}$ & Mar 1985 & $\mathrm{AL}$ & Bottenheim et al. (1986) \\
\hline $\mathrm{O}_{3}$ & & Apr 1986 & $\mathrm{AL}$ & Mickle et al. (1989) \\
\hline $\mathrm{O}_{3}$ & & $1987-1988$ & $\mathrm{mo}, \mathrm{mi}$ & Gruzdev et al. (1993) \\
\hline $\mathrm{O}_{3}$ & & $1988-1990$ & $\mathrm{BI}$ & Taalas et al. (1993) \\
\hline $\mathrm{O}_{3}$ & & 1988-1991 & ma & Taalas et al. (1993) \\
\hline $\mathrm{O}_{3}$ & & 1988-1991 & PS & Taalas et al. (1993) \\
\hline $\mathrm{O}_{3}$ & & 1989-1994 & NA & Solberg et al. (1997b) \\
\hline $\mathrm{O}_{3}$ & $14 \ldots 32 \mathrm{nmol} / \mathrm{mol}$ & Sep-Oct 1989 & $\mathrm{~mm}$ & Sturges et al. (1993d) \\
\hline $\mathrm{O}_{3}$ & $2 \ldots 48 \mathrm{nmol} / \mathrm{mol}$ & Mar-Apr 1989 & $\mathrm{BA}$ & Sturges et al. (1993c) \\
\hline $\mathrm{O}_{3}$ & & $1989-1990$ & NA & Taalas et al. (1993) \\
\hline $\mathrm{O}_{3}$ & & 1989-1990 & sy & Murayama et al. (1992) \\
\hline $\mathrm{O}_{3}$ & & 1989-1993 & NA & Solberg et al. (1996b) \\
\hline $\mathrm{O}_{3}$ & & Mar-Apr 1990 & $\mathrm{BA}$ & Sturges et al. (1993b) \\
\hline $\mathrm{O}_{3}$ & $<0.4 \ldots 20 \mathrm{nmol} / \mathrm{mol}$ & Apr 1992 & SW & Hopper et al. (1994a) \\
\hline $\mathrm{O}_{3}$ & $<0.5 \ldots 45 \mathrm{nmol} / \mathrm{mol}$ & Apr 1992 & $\mathrm{AL}$ & Anlauf et al. (1994) \\
\hline $\mathrm{O}_{3}$ & $0.9 \ldots 57.4 \mathrm{nmol} / \mathrm{mol}$ & 1992-1993 & NA & Beine (1999) \\
\hline $\mathrm{O}_{3}$ & & 1993 & $\mathrm{~nm}$ & Wessel et al. (1998) \\
\hline $\mathrm{O}_{3}$ & $0 . \ldots 72 \mathrm{nmol} / \mathrm{mol}$ & Mar-May 1993 & $\mathrm{PF}$ & Beine et al. (1996b) \\
\hline $\mathrm{O}_{3}$ & & May-Jun 1993 & $\mathrm{SU}$ & Bales et al. (1995b) \\
\hline $\mathrm{O}_{3}$ & & 1993-1994 & NA & Wessel et al. (1998) \\
\hline $\mathrm{O}_{3}$ & & 1993-2000 & $\mathrm{EU}$ & Tarasick and Bottenheim (2002) \\
\hline $\mathrm{O}_{3}$ & & 1994-1996 & $\mathrm{SC}$ & Rasmussen et al. (1997) \\
\hline $\mathrm{O}_{3}$ & & 1994-1996 & $\mathrm{TH}$ & Rasmussen et al. (1997) \\
\hline $\mathrm{O}_{3}$ & $<0.5 \ldots 36 \mathrm{nmol} / \mathrm{mol}$ & spr 1994 & NW & Ariya et al. (1998) \\
\hline $\mathrm{O}_{3}$ & & spr 1994 & $\mathrm{ARC}$ & Galaktionov et al. (1997) \\
\hline $\mathrm{O}_{3}$ & $4.0 \ldots 51.0 \mathrm{nmol} / \mathrm{mol}$ & Feb-May 1994 & NA & Beine et al. (1997a) \\
\hline $\mathrm{O}_{3}$ & $4.0 \ldots .51 .0 \mathrm{nmol} / \mathrm{mol}$ & Feb-May 1994 & NA & Beine et al. (1996a) \\
\hline $\mathrm{O}_{3}$ & bgr.: $4.0 \ldots 50 \mathrm{nmol} / \mathrm{mol}$ & Feb-May 1994 & NA & Beine et al. (1997b) \\
\hline $\mathrm{O}_{3}$ & & Mar-Apr 1994 & $\mathrm{ARC}$ & Hopper et al. (1998) \\
\hline $\mathrm{O}_{3}$ & & Mar-Jun 1994 & $\mathrm{ARC}$ & Jaeschke et al. (1997) \\
\hline $\mathrm{O}_{3}$ & $15 \ldots 45 \mathrm{nmol} / \mathrm{mol}$ & 1994-1996 & NA & Beine and Krognes (2000) \\
\hline $\mathrm{O}_{3}$ & & Apr 1994 & NW & Gong et al. (1997) \\
\hline $\mathrm{O}_{3}$ & & Jan-May 1995 & KA & Miller et al. (1997) \\
\hline $\mathrm{O}_{3}$ & & Feb-Apr 1995 & $\mathrm{AL}$ & Ariya et al. (1999) \\
\hline $\mathrm{O}_{3}$ & bgr.: $30.0 \ldots 61.6 \mathrm{nmol} / \mathrm{mol}$ & Mar-May 1995 & $\mathrm{PF}$ & Beine et al. (1997a) \\
\hline $\mathrm{O}_{3}$ & mean: $54 \mathrm{nmol} / \mathrm{mol}$ & May-Jul 1995 & $\mathrm{SU}$ & Munger et al. (1999) \\
\hline $\mathrm{O}_{3}$ & & Sep 1995 & $\mathrm{~mm}$ & Kreher et al. (1997) \\
\hline $\mathrm{O}_{3}$ & & $1995-1996$ & KA & Rasmussen et al. (1997) \\
\hline $\mathrm{O}_{3}$ & & 1995-1996 & NA & Martinez et al. (1999) \\
\hline $\mathrm{O}_{3}$ & & 1995,1996 & NA & Tuckermann et al. (1997) \\
\hline $\mathrm{O}_{3}$ & & $1995-2001$ & SN & Heidam et al. (2004) \\
\hline
\end{tabular}




\begin{tabular}{|c|c|c|c|c|}
\hline species & value & date & site & reference \\
\hline $\mathrm{O}_{3}$ & $0 \ldots 49.8 \mathrm{nmol} / \mathrm{mol}$ & $1997-1999$ (spr) & NA & Beine et al. (2001) \\
\hline $\mathrm{O}_{3}$ & & Apr-May 1998 & $\mathrm{AL}$ & Boudries and Bottenheim (2000) \\
\hline $\mathrm{O}_{3}$ & & 1998,2000 & $\mathrm{AL}$ & Sumner et al. (2002) \\
\hline $\mathrm{O}_{3}$ & $12 \ldots 21 \mathrm{nmol} / \mathrm{mol}$ & Mar 1999 & ant & Jacobi and Schrems (1999) \\
\hline $\mathrm{O}_{3}$ & $40.5 \mathrm{nmol} / \mathrm{mol}$ & sum 1999 & $\mathrm{SU}$ & Yang et al. (2002) \\
\hline $\mathrm{O}_{3}$ & & $1999-2000$ & $\mathrm{~nm}$ & Frieß et al. (2004) \\
\hline $\mathrm{O}_{3}$ & & 1999-2002 & $\mathrm{SN}$ & Skov et al. (2004) \\
\hline $\mathrm{O}_{3}$ & $51.9 \mathrm{nmol} / \mathrm{mol}$ & sum 2000 & SU & Yang et al. (2002) \\
\hline $\mathrm{O}_{3}$ & & $2000-2001$ & $\mathrm{~nm}$ & Ebinghaus et al. (2002) \\
\hline $\mathrm{O}_{3}$ & & Feb-May 2000 & $\mathrm{AL}$ & Bottenheim et al. (2002b) \\
\hline $\mathrm{O}_{3}$ & & Feb-May 2000 & $\mathrm{ARC}$ & $\begin{array}{l}\text { Ridley et al. (2003); Evans et al. } \\
(2003)\end{array}$ \\
\hline $\mathrm{O}_{3}$ & & Feb-May 2000 & $\mathrm{ARC}$ & Blake et al. (2003) \\
\hline $\mathrm{O}_{3}$ & & Jun 2000 & $\mathrm{SU}$ & Helmig et al. (2002) \\
\hline $\mathrm{O}_{3}$ & & spr 2001 & $\mathrm{HU}$ & Hönninger et al. (2004) \\
\hline $\mathrm{O}_{3}$ & mean: $14.5 \mathrm{nmol} / \mathrm{mol}$ & Dec 2001 & ant & Frey et al. (2005) \\
\hline $\mathrm{O}_{3}$ & & Mar 2002 & $\mathrm{SN}$ & Ferrari et al. (2004) \\
\hline $\mathrm{O}_{3}$ & mean: $19.3 \mathrm{nmol} / \mathrm{mol}$ & Dec 2002 & ant & Frey et al. (2005) \\
\hline $\mathrm{O}_{3}$ & mean: $27.9 \mathrm{nmol} / \mathrm{mol}$ & Jan 2003 & $\mathrm{sp}$ & Frey et al. (2005) \\
\hline $\mathrm{O}_{3}$ & & Apr-May 2003 & NA & Sprovieri et al. $(2005 a, b)$ \\
\hline $\mathrm{O}_{3}$ & $<1 \ldots 42 \mathrm{nmol} / \mathrm{mol}$ & Mar-Apr 2003 & $\mathrm{ARC}$ & Jacobi et al. (2006) \\
\hline $\mathrm{O}_{3}$ & $19 \ldots 42 \mathrm{nmol} / \mathrm{mol}$ & Mar-Apr 2003 & NA & Jacobi et al. (2006) \\
\hline $\mathrm{O}_{3}$ & & Aug-Oct 2003 & ha & Jones et al. (2006) \\
\hline $\mathrm{O}_{3}$ & $15 \ldots 58 \mathrm{nmol} / \mathrm{mol}$ & Apr-May 2004 & NA & Amoroso et al. (2005) \\
\hline $\mathrm{O}_{3}$ & & spr 2004 & $\mathrm{AL}$ & Morin et al. (2006) \\
\hline $\mathrm{O}_{3}$ & & spr 2004 & $\mathrm{AL}$ & Morin et al. (2005) \\
\hline $\mathrm{O}_{3}$ & & 2005 & $\mathrm{BA}$ & Simpson et al. (2006) \\
\hline $\mathrm{O}_{3}$ & & Jan-Apr 2005 & $\mathrm{BA}$ & Keil and Shepson (2006) \\
\hline $\mathrm{O}_{3}$ & & Mar-Apr 2005 & $\mathrm{BA}$ & Tackett et al. (2006) \\
\hline $\mathrm{OH}$ & mean: $1.1 \times 10^{5} \mathrm{~cm}^{-3}$ & Feb 1994 & $\mathrm{pa}$ & $\begin{array}{l}\text { Jefferson et al. (1998); Davis et al. } \\
(1998)\end{array}$ \\
\hline $\mathrm{OH}$ & & 2000 & $\mathrm{sp}$ & Mauldin III et al. (2004) \\
\hline $\mathrm{OH}$ & & Feb-May 2000 & $\mathrm{ARC}$ & $\begin{array}{l}\text { Ridley et al. (2003); Evans et al. } \\
(2003)\end{array}$ \\
\hline $\mathrm{OH}$ & & Feb-May 2000 & $\mathrm{ARC}$ & Mauldin III et al. (2003) \\
\hline $\mathrm{OH}$ & mean: $3.9 \times 10^{5} \mathrm{~cm}^{-3}$ & Jan-Feb 2005 & ha & Bloss et al. (2005); Lee et al. (2006) \\
\hline $\mathrm{OH}$ & noon: $7.9 \times 10^{5} \mathrm{~cm}^{-3}$ & Jan-Feb 2005 & ha & Bloss et al. (2005); Lee et al. (2006) \\
\hline $\mathrm{HO}_{2}$ & mean: $0.76 \mathrm{pmol} / \mathrm{mol}$ & Jan-Feb 2005 & ha & Bloss et al. (2005); Lee et al. (2006) \\
\hline $\mathrm{HO}_{2}$ & noon: $1.9 \mathrm{pmol} / \mathrm{mol}$ & Jan-Feb 2005 & ha & Bloss et al. (2005); Lee et al. (2006) \\
\hline $\mathrm{H}_{2} \mathrm{O}_{2}$ & $0.3 \ldots 3.5 \mathrm{nmol} / \mathrm{mol}$ & Jun-Jul 1990 & SU & Sigg et al. (1992) \\
\hline $\mathrm{H}_{2} \mathrm{O}_{2}$ & & 1992 & $\mathrm{AL}$ & de Serves (1994) \\
\hline $\mathrm{H}_{2} \mathrm{O}_{2}$ & up to $0.5 \mathrm{nmol} / \mathrm{mol}$ & 1993-1994 & ant & Fuhrer et al. (1996) \\
\hline $\mathrm{H}_{2} \mathrm{O}_{2}$ & & May-Jun 1993 & $\mathrm{SU}$ & Bales et al. (1995b) \\
\hline $\mathrm{H}_{2} \mathrm{O}_{2}$ & & May-Jul 1993 & $\mathrm{SU}$ & Bales et al. (1995a) \\
\hline $\mathrm{H}_{2} \mathrm{O}_{2}$ & & Aug 1994 & SU & Dibb et al. (1996) \\
\hline $\mathrm{H}_{2} \mathrm{O}_{2}$ & mean: $1.4 \mathrm{nmol} / \mathrm{mol}$ & Jun 1996 & $\mathrm{SU}$ & Hutterli et al. (2001) \\
\hline $\mathrm{H}_{2} \mathrm{O}_{2}$ & $<$ DL. . $0.91 \mathrm{nmol} / \mathrm{mol}$ & 1997-1999 & $\mathrm{nm}$ & Riedel et al. (2000) \\
\hline $\mathrm{H}_{2} \mathrm{O}_{2}$ & $1.78 \mathrm{nmol} / \mathrm{mol}$ & sum 1999 & $\mathrm{SU}$ & Yang et al. (2002) \\
\hline $\mathrm{H}_{2} \mathrm{O}_{2}$ & mean: $278 \mathrm{pmol} / \mathrm{mol}$ & Dec 2000 & $\mathrm{sp}$ & Hutterli et al. (2004) \\
\hline $\mathrm{H}_{2} \mathrm{O}_{2}$ & mean: $321 \mathrm{pmol} / \mathrm{mol}$ & Dec 2000 & ant & Frey et al. (2005) \\
\hline $\mathrm{H}_{2} \mathrm{O}_{2}$ & & Feb-May 2000 & $\mathrm{ARC}$ & $\begin{array}{l}\text { Ridley et al. (2003); Evans et al. } \\
(2003)\end{array}$ \\
\hline $\mathrm{H}_{2} \mathrm{O}_{2}$ & $80 \ldots 1600 \mathrm{pmol} / \mathrm{mol}$ & Jun-Jul 2000 & $\mathrm{SU}$ & Jacobi et al. (2002) \\
\hline $\mathrm{H}_{2} \mathrm{O}_{2}$ & mean: $650 \mathrm{pmol} / \mathrm{mol}$ & Dec 2001 & ant & Frey et al. (2005) \\
\hline $\mathrm{H}_{2} \mathrm{O}_{2}$ & mean: $363 \mathrm{pmol} / \mathrm{mol}$ & Dec 2002 & by & Frey et al. (2005) \\
\hline $\mathrm{H}_{2} \mathrm{O}_{2}$ & mean: $230 \mathrm{pmol} / \mathrm{mol}$ & Jan 2003 & $\mathrm{sp}$ & Frey et al. (2005) \\
\hline \multicolumn{5}{|c|}{ Nitrogen } \\
\hline $\mathrm{NH}_{3}$ & & Jan 1994 & $\mathrm{du}$ & Legrand et al. (1998) \\
\hline $\mathrm{NH}_{3}$ & & Aug 1994 & $\mathrm{SU}$ & Dibb et al. (1996) \\
\hline
\end{tabular}




\begin{tabular}{|c|c|c|c|c|}
\hline species & value & date & site & reference \\
\hline $\mathrm{NH}_{3}$ & $0 \ldots 4645.0 \mathrm{pmol} / \mathrm{mol}$ & 1997-1999 (spr) & NA & Beine et al. (2001) \\
\hline $\mathrm{N}_{2} \mathrm{O}$ & mean: $341 \mathrm{pmol} / \mathrm{mol}$ & $1988-1989$ & DY & Davidson et al. (1993b,a) \\
\hline $\mathrm{NO}$ & median: $225 \mathrm{pmol} / \mathrm{mol}$ & $1989-1999$ & $\mathrm{sp}$ & Davis et al. (2001) \\
\hline $\mathrm{NO}$ & bgr.: $0 \ldots 43.4 \mathrm{pmol} / \mathrm{mol}$ & Feb-May 1994 & NA & Beine et al. (1997a) \\
\hline $\mathrm{NO}$ & $0 \ldots 1501.8 \mathrm{pmol} / \mathrm{mol}$ & Mar-May 1995 & $\mathrm{PF}$ & Beine et al. (1997a) \\
\hline $\mathrm{NO}$ & mean: $3 \mathrm{pmol} / \mathrm{mol}$ & Jan-Mar 1997 & $\mathrm{~nm}$ & Jones et al. (1999) \\
\hline $\mathrm{NO}$ & & Jul 1998 & $\mathrm{SU}$ & Honrath et al. (1999) \\
\hline $\mathrm{NO}$ & & 1998,2000 & $\mathrm{sp}$ & Davis et al. (2004) \\
\hline $\mathrm{NO}$ & mean: $1.2 \mathrm{pmol} / \mathrm{mol}$ & Jan-Feb 1999 & $\mathrm{~nm}$ & Jacobi et al. (2000) \\
\hline $\mathrm{NO}$ & & Jul 1999 & SU & Dibb et al. (2002) \\
\hline $\mathrm{NO}$ & $24.7 \mathrm{pmol} / \mathrm{mol}$ & sum 1999 & SU & Yang et al. (2002) \\
\hline $\mathrm{NO}$ & $16.0 \mathrm{pmol} / \mathrm{mol}$ & sum 2000 & $\mathrm{SU}$ & Yang et al. (2002) \\
\hline $\mathrm{NO}$ & & Jun 2000 & $\mathrm{SU}$ & Jacobi et al. (2004) \\
\hline $\mathrm{NO}$ & & Feb-May 2000 & $\mathrm{ARC}$ & $\begin{array}{l}\text { Ridley et al. (2003); Evans et al. } \\
(2003)\end{array}$ \\
\hline $\mathrm{NO}$ & $0 \ldots 24.7 \mathrm{pmol} / \mathrm{mol}$ & Feb-May 2000 & $\mathrm{AL}$ & Beine et al. (2002) \\
\hline $\mathrm{NO}$ & & Nov-Dec 2003 & sp & Wang et al. (2006) \\
\hline $\mathrm{NO}_{2}$ & $17 \ldots 97 \mathrm{pmol} / \mathrm{mol}$ & Mar 1985 & $\mathrm{AL}$ & Bottenheim et al. (1986) \\
\hline $\mathrm{NO}_{2}$ & $85 \mathrm{pmol} / \mathrm{mol}$ & spr 1988 & $\mathrm{AL}$ & Bottenheim et al. (1990) \\
\hline $\mathrm{NO}_{2}$ & & Mar-Apr 1988 & $\mathrm{AL}$ & Bottenheim et al. (1993) \\
\hline $\mathrm{NO}_{2}$ & SCD & 1995 & $\mathrm{~mm}$ & Kreher et al. (1997) \\
\hline $\mathrm{NO}_{2}$ & & $1995-1996$ & NA & Martinez et al. (1999) \\
\hline $\mathrm{NO}_{2}$ & & 1995,1996 & NA & Tuckermann et al. (1997) \\
\hline $\mathrm{NO}_{2}$ & $0 \ldots 358.3 \mathrm{pmol} / \mathrm{mol}$ & $1997-1999(\mathrm{spr})$ & NA & Beine et al. (2001) \\
\hline $\mathrm{NO}_{2}$ & $10 \ldots 170 \mathrm{ng} / \mathrm{m}^{3}$ & May-Jun 1997 & NA & Allegrini et al. (1999) \\
\hline $\mathrm{NO}_{2}$ & $10 \ldots 300 \mathrm{ng} / \mathrm{m}^{3}$ & Dec 1997 & tn & Allegrini et al. (1999) \\
\hline $\mathrm{NO}_{2}$ & $4.94 \ldots 620.73 \mathrm{ng} / \mathrm{m}^{3}$ & 1997-1999 & tn & Ianniello et al. (2003) \\
\hline $\mathrm{NO}_{2}$ & & Jul 1998 & $\mathrm{SU}$ & Honrath et al. (1999) \\
\hline $\mathrm{NO}_{2}$ & mean: $3.2 \mathrm{pmol} / \mathrm{mol}$ & Jan-Feb 1999 & $\mathrm{~nm}$ & Jacobi et al. (2000) \\
\hline $\mathrm{NO}_{2}$ & & Jul 1999 & $\mathrm{SU}$ & Dibb et al. (2002) \\
\hline $\mathrm{NO}_{2}$ & $32.7 \mathrm{pmol} / \mathrm{mol}$ & sum 1999 & $\mathrm{SU}$ & Yang et al. (2002) \\
\hline $\mathrm{NO}_{2}$ & & Feb-May 2000 & $\mathrm{ARC}$ & $\begin{array}{l}\text { Ridley et al. (2003); Evans et al. } \\
(2003)\end{array}$ \\
\hline $\mathrm{NO}_{2}$ & $0 \ldots 38.8 \mathrm{pmol} / \mathrm{mol}$ & Feb-May 2000 & $\mathrm{AL}$ & Beine et al. (2002) \\
\hline $\mathrm{NO}_{2}$ & & Jun 2000 & $\mathrm{SU}$ & Jacobi et al. (2004) \\
\hline $\mathrm{NO}_{2}$ & $15.2 \mathrm{pmol} / \mathrm{mol}$ & sum 2000 & $\mathrm{SU}$ & Yang et al. (2002) \\
\hline $\mathrm{NO}_{2}$ & $\leq 50 \mathrm{pmol} / \mathrm{mol}$ & Apr $*$ & $\mathrm{AL}$ & Barrie and Delmas (1994) \\
\hline $\mathrm{NO}_{\mathrm{x}}$ & $\leq 30 \mathrm{pmol} / \mathrm{mol}$ & spr 1992 & $\mathrm{AL}$ & Muthuramu et al. (1994) \\
\hline $\mathrm{NO}_{\mathrm{x}}$ & $<20 \ldots 100 \mathrm{pmol} / \mathrm{mol}$ & Apr 1992 & $\mathrm{ARC}$ & Leaitch et al. (1994) \\
\hline $\mathrm{NO}_{\mathrm{x}}$ & $0 \ldots 637.5 \mathrm{pmol} / \mathrm{mol}$ & Feb-May 1994 & NA & Beine et al. (1996a) \\
\hline $\mathrm{NO}_{\mathrm{x}}$ & bgr.: $0 \ldots 143.9 \mathrm{pmol} / \mathrm{mol}$ & Feb-May 1994 & NA & Beine et al. (1997b) \\
\hline $\mathrm{NO}_{\mathrm{x}}$ & bgr.: $0 \ldots 143.9 \mathrm{pmol} / \mathrm{mol}$ & Feb-May 1994 & NA & Beine et al. (1997a) \\
\hline $\mathrm{NO}_{\mathrm{x}}$ & bgr.: $0 \ldots 955.5 \mathrm{pmol} / \mathrm{mol}$ & Mar-May 1995 & $\mathrm{PF}$ & Beine et al. (1997a) \\
\hline $\mathrm{NO}_{\mathrm{x}}$ & & 1998-1999 & $\mathrm{SU}$ & Ford et al. (2002) \\
\hline $\mathrm{NO}_{\mathrm{x}}$ & $49.4 \mathrm{pmol} / \mathrm{mol}$ & sum 1999 & $\mathrm{SU}$ & Yang et al. (2002) \\
\hline $\mathrm{NO}_{\mathrm{x}}$ & & Jun 2000 & $\mathrm{SU}$ & $\begin{array}{l}\text { Honrath et al. (2002); Jacobi et al. } \\
(2004)\end{array}$ \\
\hline $\mathrm{NO}_{\mathrm{x}}$ & $39.7 \mathrm{pmol} / \mathrm{mol}$ & sum 2000 & $\mathrm{SU}$ & Yang et al. (2002) \\
\hline $\mathrm{NO}_{\mathrm{x}}$ & & 2000 & $\mathrm{sp}$ & Oncley et al. (2004) \\
\hline $\mathrm{NO}_{\mathrm{x}}$ & & Feb-May 2000 & $\mathrm{ARC}$ & $\begin{array}{l}\text { Ridley et al. (2003); Evans et al. } \\
(2003)\end{array}$ \\
\hline $\mathrm{NO}_{\mathrm{y}}$ & & Mar-Apr 1988 & $\mathrm{AL}$ & Bottenheim et al. (1993) \\
\hline $\mathrm{NO}_{y}$ & mean: $300 \mathrm{pmol} / \mathrm{mol}$ & Feb-May 1994 & NA & Solberg et al. (1997a) \\
\hline $\mathrm{NO}_{\mathrm{y}}$ & mean: $850 \mathrm{pmol} / \mathrm{mol}$ & May-Jul 1995 & $\mathrm{SU}$ & Munger et al. (1999) \\
\hline $\mathrm{NO}_{y}$ & & 1995 & $\mathrm{SU}$ & Dibb et al. (1998) \\
\hline $\mathrm{NO}_{\mathrm{y}}$ & mean: $24 \mathrm{pmol} / \mathrm{mol}$ & Jan-Mar 1997 & $\mathrm{~nm}$ & $\begin{array}{l}\text { Jones et al. (1999); Weller et al. } \\
\text { (1999) }\end{array}$ \\
\hline $\mathrm{NO}_{y}$ & $100 \ldots 600 \mathrm{ng} / \mathrm{m}^{3}$ & May-Jun 1997 & NA & Allegrini et al. (1999) \\
\hline $\mathrm{NO}_{\mathrm{y}}$ & $300 \ldots 700 \mathrm{ng} / \mathrm{m}^{3}$ & Dec 1997 & $\operatorname{tn}$ & Allegrini et al. (1999) \\
\hline $\mathrm{NO}_{\mathrm{y}}$ & $14.58 \ldots 701.20 \mathrm{ng} / \mathrm{m}^{3}$ & 1997-1999 & tn & Ianniello et al. (2003) \\
\hline $\mathrm{NO}_{\mathrm{y}}$ & & Jul 1998 & $\mathrm{SU}$ & Honrath et al. (1999) \\
\hline
\end{tabular}




\begin{tabular}{|c|c|c|c|c|}
\hline species & value & date & site & reference \\
\hline $\mathrm{NO}_{\mathrm{y}}$ & & 1998-1999 & SU & Ford et al. (2002) \\
\hline $\mathrm{NO}_{\mathrm{y}}$ & $46 \mathrm{pmol} / \mathrm{mol}$ & $1999-2000$ & $\mathrm{~nm}$ & Weller et al. (2002) \\
\hline $\mathrm{NO}_{\mathrm{y}}$ & & Feb-Mar 2000 & $\mathrm{AL}$ & Bottenheim et al. (2002b) \\
\hline $\mathrm{NO}_{\mathrm{y}}$ & & Feb-May 2000 & $\mathrm{ARC}$ & $\begin{array}{l}\text { Ridley et al. (2003); Evans et al. } \\
(2003)\end{array}$ \\
\hline HONO & $0 \ldots \leq 240 \mathrm{pmol} / \mathrm{mol}$ & Apr 1992 & $\mathrm{AL}$ & Hausmann and Platt (1994) \\
\hline HONO & $<1.7 \ldots 68 \mathrm{pmol} / \mathrm{mol}$ & win 1992 & $\mathrm{AL}$ & Li (1994) \\
\hline HONO & $<1.7 \ldots 20 \mathrm{pmol} / \mathrm{mol}$ & spr 1992 & $\mathrm{AL}$ & $\mathrm{Li}(1994)$ \\
\hline HONO & $0 \ldots 64.8 \mathrm{pmol} / \mathrm{mol}$ & $1997-1999(\mathrm{spr})$ & NA & Beine et al. (2001) \\
\hline HONO & & Jul 1999 & SU & Dibb et al. (2002) \\
\hline HONO & $7.24 \mathrm{pmol} / \mathrm{mol}$ & sum 1999 & SU & Yang et al. (2002) \\
\hline HONO & $12.7 \mathrm{pmol} / \mathrm{mol}$ & sum 2000 & $\mathrm{SU}$ & Yang et al. (2002) \\
\hline HONO & & 2000 & $\mathrm{sp}$ & Dibb et al. (2004) \\
\hline HONO & & Feb-May 2000 & $\mathrm{AL}$ & Zhou et al. (2001) \\
\hline HONO & & Jun 2000 & $\mathrm{SU}$ & $\begin{array}{l}\text { Honrath et al. (2002); Jacobi et al. } \\
(2004)\end{array}$ \\
\hline HONO & $<$ DL. . .20 pmol $/ \mathrm{mol}$ & Feb-May 2001 & NA & Beine et al. (2003) \\
\hline HONO & $0 \ldots 48.3 \mathrm{pmol} / \mathrm{mol}$ & Apr-May 2004 & NA & Amoroso et al. (2005) \\
\hline HONO & $0 \ldots 7 \mathrm{pmol} / \mathrm{mol}$ & Nov 2004 & $\mathrm{bp}$ & Beine et al. (2006) \\
\hline HONO & $\begin{array}{l}10.1 \mathrm{pmol} / \mathrm{mol}(\mathrm{sum}), \quad 0.8 \\
\mathrm{pmol} / \mathrm{mol} \text { (win) }\end{array}$ & $2004-2005$ & ha & Clemitshaw et al. (2006) \\
\hline $\mathrm{HNO}_{3}(\mathrm{~g}+\mathrm{aq})$ & $24 \ldots 72 \mathrm{pmol} / \mathrm{mol}$ & Mar 1985 & $\mathrm{AL}$ & Bottenheim et al. (1986) \\
\hline $\mathrm{HNO}_{3}$ & $3.5 \ldots 180 \mathrm{pmol} / \mathrm{mol}$ & spr 1988 & $\mathrm{AL}$ & Bottenheim et al. (1990) \\
\hline $\mathrm{HNO}_{3}$ & & Mar-Apr 1988 & $\mathrm{AL}$ & Bottenheim et al. (1993) \\
\hline $\mathrm{HNO}_{3}$ & mean: $77 \mathrm{ng} / \mathrm{m}^{3}$ & May 1989 & $\mathrm{AL}$ & Kieser et al. (1993) \\
\hline $\mathrm{HNO}_{3}(\mathrm{~g}+\mathrm{aq})$ & & $1990-2001$ & $\mathrm{SN}$ & Heidam et al. (2004) \\
\hline $\mathrm{HNO}_{3}$ & & Jan-Feb 1991 & $\operatorname{tn}$ & Allegrini et al. (1994) \\
\hline $\mathrm{HNO}_{3}$ & $5 \ldots 100 \mathrm{pmol} / \mathrm{mol}$ & win/spr 1992 & $\mathrm{AL}$ & Barrie et al. (1994a) \\
\hline $\mathrm{HNO}_{3}$ & $\leq 40 \ldots 110 \mathrm{pmol} / \mathrm{mol}$ & Apr 1992 & $\mathrm{ARC}$ & Leaitch et al. (1994) \\
\hline $\mathrm{HNO}_{3}$ & mean: $0.9 \mathrm{nmol} / \mathrm{m}^{3}(\mathrm{STP})$ & Jun-Jul 1993 & SU & Dibb et al. (1994) \\
\hline $\mathrm{HNO}_{3}$ & & 1994-1995 & $\mathrm{SU}$ & Dibb et al. (1998) \\
\hline $\mathrm{HNO}_{3}$ & & Mar-Jun 1994 & $\mathrm{ARC}$ & Jaeschke et al. (1997) \\
\hline $\mathrm{HNO}_{3}$ & & Aug 1994 & SU & Dibb et al. (1996) \\
\hline $\mathrm{HNO}_{3}$ & mean: $5 \mathrm{pmol} / \mathrm{mol}$ & Jan-Mar 1997 & $\mathrm{~nm}$ & $\begin{array}{l}\text { Jones et al. (1999); Weller et al. } \\
(1999)\end{array}$ \\
\hline $\mathrm{HNO}_{3}$ & $0 . \ldots 229.1 \mathrm{pmol} / \mathrm{mol}$ & 1997-1999 (spr) & NA & Beine et al. (2001) \\
\hline $\mathrm{HNO}_{3}$ & mean: $4.0 \mathrm{pmol} / \mathrm{mol}$ & Jan-Feb 1999 & $\mathrm{~nm}$ & Jacobi et al. (2000) \\
\hline $\mathrm{HNO}_{3}$ & & $2000-2001$ & $\mathrm{du}$ & Jourdain and Legrand (2002) \\
\hline $\mathrm{HNO}_{3}$ & mean: $14.5 \mathrm{ng} / \mathrm{m}^{3}$ & Feb 2000 & $\mathrm{AL}$ & Ianniello et al. (2002) \\
\hline $\mathrm{HNO}_{3}$ & & Mar-Apr 2000 & NA & Hara et al. (2002b) \\
\hline $\mathrm{HNO}_{3}$ & mean: $54.9 \mathrm{ng} / \mathrm{m}^{3}$ & Apr-May 2000 & $\mathrm{AL}$ & Ianniello et al. (2002) \\
\hline $\mathrm{HNO}_{3}$ & & Jun 2000 & $\mathrm{SU}$ & $\begin{array}{l}\text { Honrath et al. (2002); Jacobi et al. } \\
(2004)\end{array}$ \\
\hline $\mathrm{HNO}_{3}$ & & 2000 & $\mathrm{sp}$ & Dibb et al. (2004) \\
\hline $\mathrm{HNO}_{3}$ & & 2000 & $\mathrm{sp}$ & Huey et al. (2004) \\
\hline $\mathrm{HNO}_{3}$ & $0.9 \ldots 70 \mathrm{pmol} / \mathrm{mol}$ & Feb-May 2001 & NA & Beine et al. (2003) \\
\hline $\mathrm{HNO}_{3}$ & & Nov-Dec 2003 & sp & Wang et al. (2006) \\
\hline $\mathrm{HNO}_{4}$ & & Feb-May 2000 & $\mathrm{AL}$ & Zhou et al. (2001) \\
\hline $\mathrm{HNO}_{4}$ & mean: $25 \mathrm{pmol} / \mathrm{mol}$ & Dec 2000 & $\mathrm{sp}$ & Slusher et al. (2002) \\
\hline $\mathrm{HNO}_{4}$ & & Nov-Dec 2003 & $\mathrm{sp}$ & Wang et al. (2006) \\
\hline \multicolumn{5}{|c|}{ Organic: C, H (alkanes) } \\
\hline $\mathrm{CH}_{4}$ & & Apr 1986 & $\mathrm{AL}$ & Trivett et al. (1989) \\
\hline $\mathrm{CH}_{4}$ & mean: $1780 \mathrm{nmol} / \mathrm{mol}$ & $1988-1989$ & DY & Davidson et al. (1993b,a) \\
\hline $\mathrm{CH}_{4}$ & & $1989-1990$ & $\mathrm{AL}$ & Hopper et al. (1994b) \\
\hline $\mathrm{CH}_{4}$ & $1800 \ldots 1950 \mathrm{nmol} / \mathrm{mol}$ & win/spr 1992 & $\mathrm{AL}$ & Worthy et al. (1994) \\
\hline $\mathrm{CH}_{4}$ & & Apr 1994 & $\mathrm{AL}, \mathrm{NW}$ & Ariya et al. (1998) \\
\hline $\mathrm{CH}_{4}$ & & Feb-Apr 1995 & $\mathrm{AL}$ & Ariya et al. (1999) \\
\hline $\mathrm{CH}_{4}$ & $1800 \mathrm{nmol} / \mathrm{mol}$ & sum 1999 & $\mathrm{SU}$ & Yang et al. (2002) \\
\hline $\mathrm{CH}_{4}$ & & Feb-May 2000 & $\mathrm{ARC}$ & $\begin{array}{l}\text { Ridley et al. (2003); Evans et al. } \\
(2003)\end{array}$ \\
\hline
\end{tabular}




\begin{tabular}{|c|c|c|c|c|}
\hline species & value & date & site & reference \\
\hline $\mathrm{CH}_{4}$ & $1816 \mathrm{nmol} / \mathrm{mol}$ & sum 2000 & $\mathrm{SU}$ & Yang et al. (2002) \\
\hline $\mathrm{C}_{2} \mathrm{H}_{6}$ & & 1982,1983 & $\mathrm{NO}$ & Hov et al. (1984) \\
\hline $\mathrm{C}_{2} \mathrm{H}_{6}$ & mean: $370 \mathrm{pmol} / \mathrm{mol}$ & $1982-1985$ & $\mathrm{~nm}$ & Rudolph et al. (1989) \\
\hline $\mathrm{C}_{2} \mathrm{H}_{6}$ & & $1983-1986$ & NA & Hov et al. (1989) \\
\hline $\mathrm{C}_{2} \mathrm{H}_{6}$ & & 1989-1994 & NA & Solberg et al. (1996a) \\
\hline $\mathrm{C}_{2} \mathrm{H}_{6}$ & $2000 \ldots 4300 \mathrm{pmol} / \mathrm{mol}$ & Mar 1989 & $\mathrm{BA}$ & Doskey and Gaffney (1992) \\
\hline $\mathrm{C}_{2} \mathrm{H}_{6}$ & mean: $853 \mathrm{pmol} / \mathrm{mol}$ & Apr 1989 & $\mathrm{AL}$ & Kieser et al. (1993) \\
\hline $\mathrm{C}_{2} \mathrm{H}_{6}$ & mean: $683 \mathrm{pmol} / \mathrm{mol}$ & May 1989 & $\mathrm{AL}$ & Kieser et al. (1993) \\
\hline $\mathrm{C}_{2} \mathrm{H}_{6}$ & $1500 \ldots 4000 \mathrm{pmol} / \mathrm{mol}$ & win/spr 1992 & $\mathrm{AL}$ & Jobson et al. (1994) \\
\hline $\mathrm{C}_{2} \mathrm{H}_{6}$ & & Mar-Jun 1993 & NA & Solberg et al. (1996b) \\
\hline $\mathrm{C}_{2} \mathrm{H}_{6}$ & & Apr 1994 & $\mathrm{AL}, \mathrm{NW}$ & Ariya et al. (1998) \\
\hline $\mathrm{C}_{2} \mathrm{H}_{6}$ & & Feb-Apr 1995 & $\mathrm{AL}$ & Ariya et al. (1999) \\
\hline $\mathrm{C}_{2} \mathrm{H}_{6}$ & $1366 \ldots 2594 \mathrm{pmol} / \mathrm{mol}$ & Mar-May 1995 & $\mathrm{PF}$ & Herring et al. (1997) \\
\hline $\mathrm{C}_{2} \mathrm{H}_{6}$ & & $1997-1998$ & $\mathrm{SU}$ & Swanson et al. (2003) \\
\hline $\mathrm{C}_{2} \mathrm{H}_{6}$ & & Apr-May 1998 & $\mathrm{AL}$ & Boudries and Bottenheim (2000) \\
\hline $\mathrm{C}_{2} \mathrm{H}_{6}$ & & 1998,2000 & $\mathrm{AL}$ & Bottenheim et al. (2002a) \\
\hline $\mathrm{C}_{2} \mathrm{H}_{6}$ & & Feb-May 2000 & $\mathrm{ARC}$ & $\begin{array}{l}\text { Ridley et al. (2003); Evans et al. } \\
(2003)\end{array}$ \\
\hline $\mathrm{C}_{2} \mathrm{H}_{6}$ & & Feb-May 2000 & $\mathrm{ARC}$ & Blake et al. (2003) \\
\hline $\mathrm{C}_{2} \mathrm{H}_{6}$ & mean: $186 \mathrm{pmol} / \mathrm{mol}$ & $2004-2005$ & ha & Read et al. (2006) \\
\hline $\mathrm{C}_{3} \mathrm{H}_{8}$ & & 1982,1983 & $\mathrm{NO}$ & Hov et al. (1984) \\
\hline $\mathrm{C}_{3} \mathrm{H}_{8}$ & mean: $70 \mathrm{pmol} / \mathrm{mol}$ & $1982-1985$ & $\mathrm{~nm}$ & Rudolph et al. (1989) \\
\hline $\mathrm{C}_{3} \mathrm{H}_{8}$ & & $1983-1986$ & NA & Hov et al. (1989) \\
\hline $\mathrm{C}_{3} \mathrm{H}_{8}$ & & 1989-1994 & NA & Solberg et al. (1996a) \\
\hline $\mathrm{C}_{3} \mathrm{H}_{8}$ & $800 \ldots 2200 \mathrm{pmol} / \mathrm{mol}$ & Mar 1989 & $\mathrm{BA}$ & Doskey and Gaffney (1992) \\
\hline $\mathrm{C}_{3} \mathrm{H}_{8}$ & mean: $505 \mathrm{pmol} / \mathrm{mol}$ & Apr 1989 & $\mathrm{AL}$ & Kieser et al. (1993) \\
\hline $\mathrm{C}_{3} \mathrm{H}_{8}$ & mean: $288 \mathrm{pmol} / \mathrm{mol}$ & May 1989 & $\mathrm{AL}$ & Kieser et al. (1993) \\
\hline $\mathrm{C}_{3} \mathrm{H}_{8}$ & $500 \ldots 3000 \mathrm{pmol} / \mathrm{mol}$ & win/spr 1992 & $\mathrm{AL}$ & Jobson et al. (1994) \\
\hline $\mathrm{C}_{3} \mathrm{H}_{8}$ & & Mar-Jun 1993 & NA & Solberg et al. (1996b) \\
\hline $\mathrm{C}_{3} \mathrm{H}_{8}$ & & Apr 1994 & $\mathrm{AL}, \mathrm{NW}$ & Ariya et al. (1998) \\
\hline $\mathrm{C}_{3} \mathrm{H}_{8}$ & & Feb-Apr 1995 & $\mathrm{AL}$ & Ariya et al. (1999) \\
\hline $\mathrm{C}_{3} \mathrm{H}_{8}$ & $188 \ldots 1542 \mathrm{pmol} / \mathrm{mol}$ & Mar-May 1995 & $\mathrm{PF}$ & Herring et al. (1997) \\
\hline $\mathrm{C}_{3} \mathrm{H}_{8}$ & & 1997-1998 & $\mathrm{SU}$ & Swanson et al. (2003) \\
\hline $\mathrm{C}_{3} \mathrm{H}_{8}$ & & Apr-May 1998 & $\mathrm{AL}$ & Boudries and Bottenheim (2000) \\
\hline $\mathrm{C}_{3} \mathrm{H}_{8}$ & & 1998,2000 & $\mathrm{AL}$ & Bottenheim et al. (2002a) \\
\hline $\mathrm{C}_{3} \mathrm{H}_{8}$ & & Feb-May 2000 & $\mathrm{ARC}$ & $\begin{array}{l}\text { Ridley et al. (2003); Evans et al. } \\
(2003)\end{array}$ \\
\hline $\mathrm{C}_{3} \mathrm{H}_{8}$ & & Feb-May 2000 & $\mathrm{ARC}$ & Blake et al. (2003) \\
\hline $\mathrm{C}_{3} \mathrm{H}_{8}$ & mean: $31 \mathrm{pmol} / \mathrm{mol}$ & $2004-2005$ & ha & Read et al. (2006) \\
\hline $\mathrm{n}-\mathrm{C}_{4} \mathrm{H}_{10}$ & & 1982,1983 & $\mathrm{NO}$ & Hov et al. (1984) \\
\hline $\mathrm{n}-\mathrm{C}_{4} \mathrm{H}_{10}$ & & $1983-1986$ & NA & Hov et al. (1989) \\
\hline $\mathrm{n}-\mathrm{C}_{4} \mathrm{H}_{10}$ & $460 \ldots 910 \mathrm{pmol} / \mathrm{mol}$ & Mar 1989 & $\mathrm{BA}$ & Doskey and Gaffney (1992) \\
\hline $\mathrm{n}-\mathrm{C}_{4} \mathrm{H}_{10}$ & mean: $167 \mathrm{pmol} / \mathrm{mol}$ & Apr 1989 & $\mathrm{AL}$ & Kieser et al. (1993) \\
\hline $\mathrm{n}-\mathrm{C}_{4} \mathrm{H}_{10}$ & mean: $60 \mathrm{pmol} / \mathrm{mol}$ & May 1989 & $\mathrm{AL}$ & Kieser et al. (1993) \\
\hline $\mathrm{n}-\mathrm{C}_{4} \mathrm{H}_{10}$ & & 1989-1994 & NA & Solberg et al. (1996a) \\
\hline $\mathrm{n}-\mathrm{C}_{4} \mathrm{H}_{10}$ & $100 \ldots 1500 \mathrm{pmol} / \mathrm{mol}$ & win/spr 1992 & $\mathrm{AL}$ & Jobson et al. (1994) \\
\hline $\mathrm{n}-\mathrm{C}_{4} \mathrm{H}_{10}$ & $19.6 \ldots 126.2 \mathrm{pmol} / \mathrm{mol}$ & Apr 1992 & $\mathrm{AL}$ & Yokouchi et al. (1994) \\
\hline $\mathrm{n}-\mathrm{C}_{4} \mathrm{H}_{10}$ & & Mar-Jun 1993 & NA & Solberg et al. (1996b) \\
\hline $\mathrm{n}-\mathrm{C}_{4} \mathrm{H}_{10}$ & & Apr 1994 & $\mathrm{AL}, \mathrm{NW}$ & Ariya et al. (1998) \\
\hline $\mathrm{n}-\mathrm{C}_{4} \mathrm{H}_{10}$ & & Feb-Apr 1995 & $\mathrm{AL}$ & Ariya et al. (1999) \\
\hline $\mathrm{n}-\mathrm{C}_{4} \mathrm{H}_{10}$ & $22 \ldots 912 \mathrm{pmol} / \mathrm{mol}$ & Mar-May 1995 & $\mathrm{PF}$ & Herring et al. (1997) \\
\hline $\mathrm{n}-\mathrm{C}_{4} \mathrm{H}_{10}$ & & $1997-1998$ & $\mathrm{SU}$ & Swanson et al. (2003) \\
\hline $\mathrm{n}-\mathrm{C}_{4} \mathrm{H}_{10}$ & & Apr-May 1998 & $\mathrm{AL}$ & Boudries and Bottenheim (2000) \\
\hline $\mathrm{n}-\mathrm{C}_{4} \mathrm{H}_{10}$ & & 1998,2000 & $\mathrm{AL}$ & Bottenheim et al. (2002a) \\
\hline $\mathrm{n}-\mathrm{C}_{4} \mathrm{H}_{10}$ & & Feb-May 2000 & $\mathrm{ARC}$ & $\begin{array}{l}\text { Ridley et al. (2003); Evans et al. } \\
(2003)\end{array}$ \\
\hline $\mathrm{n}-\mathrm{C}_{4} \mathrm{H}_{10}$ & & Feb-May 2000 & $\mathrm{ARC}$ & Blake et al. (2003) \\
\hline $\mathrm{n}-\mathrm{C}_{4} \mathrm{H}_{10}$ & mean: $4.9 \mathrm{pmol} / \mathrm{mol}$ & $2004-2005$ & ha & Read et al. (2006) \\
\hline $\mathrm{n}-\mathrm{C}_{4} \mathrm{H}_{10}$ & & Mar-Apr 2005 & $\mathrm{BA}$ & Tackett et al. (2006) \\
\hline $\mathrm{i}-\mathrm{C}_{4} \mathrm{H}_{10}$ & & 1982,1983 & $\mathrm{NO}$ & Hov et al. (1984) \\
\hline $\mathrm{i}-\mathrm{C}_{4} \mathrm{H}_{10}$ & & $1983-1986$ & NA & Hov et al. (1989) \\
\hline
\end{tabular}




\begin{tabular}{|c|c|c|c|c|}
\hline species & value & date & site & reference \\
\hline $\mathrm{i}-\mathrm{C}_{4} \mathrm{H}_{10}$ & & $1989-1994$ & NA & Solberg et al. (1996a) \\
\hline $\mathrm{i}-\mathrm{C}_{4} \mathrm{H}_{10}$ & $140 \ldots 350 \mathrm{pmol} / \mathrm{mol}$ & Mar 1989 & $\mathrm{BA}$ & Doskey and Gaffney (1992) \\
\hline $\mathrm{i}-\mathrm{C}_{4} \mathrm{H}_{10}$ & mean: $459 \mathrm{pmol} / \mathrm{mol}$ & Apr 1989 & $\mathrm{AL}$ & Kieser et al. (1993) \\
\hline $\mathrm{i}-\mathrm{C}_{4} \mathrm{H}_{10}$ & mean: $344 \mathrm{pmol} / \mathrm{mol}$ & May 1989 & $\mathrm{AL}$ & Kieser et al. (1993) \\
\hline $\mathrm{i}-\mathrm{C}_{4} \mathrm{H}_{10}$ & $100 \ldots 800 \mathrm{pmol} / \mathrm{mol}$ & win/spr 1992 & $\mathrm{AL}$ & Jobson et al. (1994) \\
\hline $\mathrm{i}-\mathrm{C}_{4} \mathrm{H}_{10}$ & & Apr 1994 & $\mathrm{AL}, \mathrm{NW}$ & Ariya et al. (1998) \\
\hline $\mathrm{i}-\mathrm{C}_{4} \mathrm{H}_{10}$ & & Feb-Apr 1995 & $\mathrm{AL}$ & Ariya et al. (1999) \\
\hline $\mathrm{i}-\mathrm{C}_{4} \mathrm{H}_{10}$ & $12 \ldots 525 \mathrm{pmol} / \mathrm{mol}$ & Mar-May 1995 & $\mathrm{PF}$ & Herring et al. (1997) \\
\hline $\mathrm{i}-\mathrm{C}_{4} \mathrm{H}_{10}$ & & $1997-1998$ & $\mathrm{SU}$ & Swanson et al. (2003) \\
\hline $\mathrm{i}-\mathrm{C}_{4} \mathrm{H}_{10}$ & & Apr-May 1998 & $\mathrm{AL}$ & Boudries and Bottenheim (2000) \\
\hline $\mathrm{i}-\mathrm{C}_{4} \mathrm{H}_{10}$ & & 1998,2000 & $\mathrm{AL}$ & Bottenheim et al. (2002a) \\
\hline $\mathrm{i}-\mathrm{C}_{4} \mathrm{H}_{10}$ & & Feb-May 2000 & $\mathrm{ARC}$ & $\begin{array}{l}\text { Ridley et al. (2003); Evans et al. } \\
(2003)\end{array}$ \\
\hline $\mathrm{i}-\mathrm{C}_{4} \mathrm{H}_{10}$ & mean: $3.2 \mathrm{pmol} / \mathrm{mol}$ & 2004-2005 & ha & Read et al. (2006) \\
\hline $\mathrm{n}-\mathrm{C}_{5} \mathrm{H}_{12}$ & & 1982,1983 & $\mathrm{NO}$ & Hov et al. (1984) \\
\hline $\mathrm{n}-\mathrm{C}_{5} \mathrm{H}_{12}$ & & $1983-1986$ & NA & Hov et al. (1989) \\
\hline $\mathrm{n}-\mathrm{C}_{5} \mathrm{H}_{12}$ & & 1989-1994 & NA & Solberg et al. (1996a) \\
\hline $\mathrm{n}-\mathrm{C}_{5} \mathrm{H}_{12}$ & $140 \ldots 330 \mathrm{pmol} / \mathrm{mol}$ & Mar 1989 & $\mathrm{BA}$ & Doskey and Gaffney (1992) \\
\hline $\mathrm{n}-\mathrm{C}_{5} \mathrm{H}_{12}$ & $20 \ldots 600 \mathrm{pmol} / \mathrm{mol}$ & win/spr 1992 & $\mathrm{AL}$ & Jobson et al. (1994) \\
\hline $\mathrm{n}-\mathrm{C}_{5} \mathrm{H}_{12}$ & & Apr 1994 & AL, NW & Ariya et al. (1998) \\
\hline $\mathrm{n}-\mathrm{C}_{5} \mathrm{H}_{12}$ & & Feb-Apr 1995 & $\mathrm{AL}$ & Ariya et al. (1999) \\
\hline $\mathrm{n}-\mathrm{C}_{5} \mathrm{H}_{12}$ & & Apr-May 1998 & $\mathrm{AL}$ & Boudries and Bottenheim (2000) \\
\hline $\mathrm{n}-\mathrm{C}_{5} \mathrm{H}_{12}$ & & 1998,2000 & $\mathrm{AL}$ & Bottenheim et al. (2002a) \\
\hline $\mathrm{n}-\mathrm{C}_{5} \mathrm{H}_{12}$ & & Feb-May 2000 & $\mathrm{ARC}$ & $\begin{array}{l}\text { Ridley et al. (2003); Evans et al. } \\
(2003)\end{array}$ \\
\hline $\mathrm{i}-\mathrm{C}_{5} \mathrm{H}_{12}$ & & 1982,1983 & $\mathrm{NO}$ & Hov et al. (1984) \\
\hline $\mathrm{i}-\mathrm{C}_{5} \mathrm{H}_{12}$ & & 1983-1986 & $\mathrm{NA}$ & Hov et al. (1989) \\
\hline $\mathrm{i}-\mathrm{C}_{5} \mathrm{H}_{12}$ & & 1989-1994 & NA & Solberg et al. (1996a) \\
\hline $\mathrm{i}-\mathrm{C}_{5} \mathrm{H}_{12}$ & $76 \ldots 220 \mathrm{pmol} / \mathrm{mol}$ & Mar 1989 & $\mathrm{BA}$ & Doskey and Gaffney (1992) \\
\hline $\mathrm{i}-\mathrm{C}_{5} \mathrm{H}_{12}$ & $20 \ldots 600 \mathrm{pmol} / \mathrm{mol}$ & win/spr 1992 & $\mathrm{AL}$ & Jobson et al. (1994) \\
\hline $\mathrm{i}-\mathrm{C}_{5} \mathrm{H}_{12}$ & $7.2 \ldots 50.3 \mathrm{pmol} / \mathrm{mol}$ & Apr 1992 & $\mathrm{AL}$ & Yokouchi et al. (1994) \\
\hline $\mathrm{i}-\mathrm{C}_{5} \mathrm{H}_{12}$ & & Apr 1994 & $\mathrm{AL}, \mathrm{NW}$ & Ariya et al. (1998) \\
\hline $\mathrm{i}-\mathrm{C}_{5} \mathrm{H}_{12}$ & & Feb-Apr 1995 & $\mathrm{AL}$ & Ariya et al. (1999) \\
\hline $\mathrm{i}-\mathrm{C}_{5} \mathrm{H}_{12}$ & & Apr-May 1998 & $\mathrm{AL}$ & Boudries and Bottenheim (2000) \\
\hline $\mathrm{i}-\mathrm{C}_{5} \mathrm{H}_{12}$ & & 1998,2000 & $\mathrm{AL}$ & Bottenheim et al. (2002a) \\
\hline $\mathrm{i}-\mathrm{C}_{5} \mathrm{H}_{12}$ & & Feb-May 2000 & $\mathrm{ARC}$ & $\begin{array}{l}\text { Ridley et al. (2003); Evans et al. } \\
(2003)\end{array}$ \\
\hline dimethylpropane & & $1983-1986$ & NA & Hov et al. (1989) \\
\hline $\mathrm{n}-\mathrm{C}_{6} \mathrm{H}_{14}$ & & 1982,1983 & $\mathrm{NO}$ & Hov et al. (1984) \\
\hline $\mathrm{n}-\mathrm{C}_{6} \mathrm{H}_{14}$ & & $1983-1986$ & NA & Hov et al. (1989) \\
\hline $\mathrm{n}-\mathrm{C}_{6} \mathrm{H}_{14}$ & $35 \ldots 139 \mathrm{pmol} / \mathrm{mol}$ & Mar 1989 & $\mathrm{BA}$ & Doskey and Gaffney (1992) \\
\hline $\mathrm{n}-\mathrm{C}_{6} \mathrm{H}_{14}$ & $7 \ldots 200 \mathrm{pmol} / \mathrm{mol}$ & win/spr 1992 & $\mathrm{AL}$ & Jobson et al. (1994) \\
\hline $\mathrm{n}-\mathrm{C}_{6} \mathrm{H}_{14}$ & & Apr 1994 & AL, NW & Ariya et al. (1998) \\
\hline $\mathrm{n}-\mathrm{C}_{6} \mathrm{H}_{14}$ & & Feb-Apr 1995 & $\mathrm{AL}$ & Ariya et al. (1999) \\
\hline $\mathrm{n}-\mathrm{C}_{6} \mathrm{H}_{14}$ & & Feb-May 2000 & $\mathrm{ARC}$ & $\begin{array}{l}\text { Ridley et al. (2003); Evans et al. } \\
(2003)\end{array}$ \\
\hline 2-methylpentane & & 1982,1983 & $\mathrm{NO}$ & Hov et al. (1984) \\
\hline 2-methylpentane & & 1983-1986 & NA & Hov et al. (1989) \\
\hline 2-methylpentane & 7...200 pmol $/ \mathrm{mol}$ & win/spr 1992 & $\mathrm{AL}$ & Jobson et al. (1994) \\
\hline 2-methylpentane & & Apr 1994 & $\mathrm{AL}, \mathrm{NW}$ & Ariya et al. (1998) \\
\hline 2-methylpentane & & Feb-Apr 1995 & $\mathrm{AL}$ & Ariya et al. (1999) \\
\hline 3-methylpentane & & 1982,1983 & $\mathrm{NO}$ & Hov et al. (1984) \\
\hline 3-methylpentane & & $1983-1986$ & NA & Hov et al. (1989) \\
\hline 3-methylpentane & $42 \ldots 98 \mathrm{pmol} / \mathrm{mol}$ & Mar 1989 & $\mathrm{BA}$ & Doskey and Gaffney (1992) \\
\hline 3-methylpentane & & Apr 1994 & $\mathrm{AL}, \mathrm{NW}$ & Ariya et al. (1998) \\
\hline 3-methylpentane & & Feb-Apr 1995 & $\mathrm{AL}$ & Ariya et al. (1999) \\
\hline 2,2-dimethylbutane & & $1983-1986$ & $\mathrm{NA}$ & Hov et al. (1989) \\
\hline 2,2-dimethylbutane & $<2 \ldots 82 \mathrm{pmol} / \mathrm{mol}$ & Mar 1989 & $\mathrm{BA}$ & Doskey and Gaffney (1992) \\
\hline cyclohexane & & 1982,1983 & $\mathrm{NO}$ & Hov et al. (1984) \\
\hline methylcyclopentane & $23 \ldots 60 \mathrm{pmol} / \mathrm{mol}$ & Mar 1989 & $\mathrm{BA}$ & Doskey and Gaffney (1992) \\
\hline
\end{tabular}




\begin{tabular}{|c|c|c|c|c|}
\hline species & value & date & site & reference \\
\hline $\mathrm{n}-\mathrm{C}_{7} \mathrm{H}_{16}$ & $14 \ldots 50 \mathrm{pmol} / \mathrm{mol}$ & Mar 1989 & $\mathrm{BA}$ & Doskey and Gaffney (1992) \\
\hline $\mathrm{n}-\mathrm{C}_{7} \mathrm{H}_{16}$ & & Apr 1994 & $\mathrm{AL}, \mathrm{NW}$ & Ariya et al. (1998) \\
\hline $\mathrm{n}-\mathrm{C}_{7} \mathrm{H}_{16}$ & & Feb-Apr 1995 & $\mathrm{AL}$ & Ariya et al. (1999) \\
\hline methylhexane & $24 \ldots 60 \mathrm{pmol} / \mathrm{mol}$ & Mar 1989 & $\mathrm{BA}$ & Doskey and Gaffney (1992) \\
\hline \multicolumn{5}{|c|}{ Organic: C, H (unsaturated) } \\
\hline $\mathrm{C}_{2} \mathrm{H}_{4}$ & mean: $360 \mathrm{pmol} / \mathrm{mol}$ & $1982-1985$ & $\mathrm{~nm}$ & Rudolph et al. (1989) \\
\hline $\mathrm{C}_{2} \mathrm{H}_{4}$ & & 1982,1983 & $\mathrm{NO}$ & Hov et al. (1984) \\
\hline $\mathrm{C}_{2} \mathrm{H}_{4}$ & & 1983-1986 & NA & Hov et al. (1989) \\
\hline $\mathrm{C}_{2} \mathrm{H}_{4}$ & & 1989-1994 & NA & Solberg et al. (1996a) \\
\hline $\mathrm{C}_{2} \mathrm{H}_{4}$ & $330 \ldots 1000 \mathrm{pmol} / \mathrm{mol}$ & Mar 1989 & $\mathrm{BA}$ & Doskey and Gaffney (1992) \\
\hline $\mathrm{C}_{2} \mathrm{H}_{4}$ & mean: $40 \mathrm{pmol} / \mathrm{mol}$ & Apr 1989 & $\mathrm{AL}$ & Kieser et al. (1993) \\
\hline $\mathrm{C}_{2} \mathrm{H}_{4}$ & $0 \ldots 1000 \mathrm{pmol} / \mathrm{mol}$ & win/spr 1992 & $\mathrm{AL}$ & Jobson et al. (1994) \\
\hline $\mathrm{C}_{2} \mathrm{H}_{4}$ & & Mar-Jun 1993 & NA & Solberg et al. (1996b) \\
\hline $\mathrm{C}_{2} \mathrm{H}_{4}$ & $23 \ldots 509 \mathrm{pmol} / \mathrm{mol}$ & Mar-May 1995 & $\mathrm{PF}$ & Herring et al. (1997) \\
\hline $\mathrm{C}_{2} \mathrm{H}_{4}$ & mean: $26 \mathrm{pmol} / \mathrm{mol}$ & Mar-May 1996 & NA & Ramacher et al. (1999) \\
\hline $\mathrm{C}_{2} \mathrm{H}_{4}$ & & 1998,2000 & $\mathrm{AL}$ & Bottenheim et al. (2002a) \\
\hline $\mathrm{C}_{3} \mathrm{H}_{6}$ & & 1982,1983 & $\mathrm{NO}$ & Hov et al. (1984) \\
\hline $\mathrm{C}_{3} \mathrm{H}_{6}$ & mean: $210 \mathrm{pmol} / \mathrm{mol}$ & $1982-1985$ & $\mathrm{~nm}$ & Rudolph et al. (1989) \\
\hline $\mathrm{C}_{3} \mathrm{H}_{6}$ & & 1983-1986 & NA & Hov et al. (1989) \\
\hline $\mathrm{C}_{3} \mathrm{H}_{6}$ & & 1989-1994 & NA & Solberg et al. (1996a) \\
\hline $\mathrm{C}_{3} \mathrm{H}_{6}$ & $160 \ldots 330 \mathrm{pmol} / \mathrm{mol}$ & Mar 1989 & $\mathrm{BA}$ & Doskey and Gaffney (1992) \\
\hline $\mathrm{C}_{3} \mathrm{H}_{6}$ & mean: $94 \mathrm{pmol} / \mathrm{mol}$ & Apr 1989 & $\mathrm{AL}$ & Kieser et al. (1993) \\
\hline $\mathrm{C}_{3} \mathrm{H}_{6}$ & mean: $117 \mathrm{pmol} / \mathrm{mol}$ & May 1989 & $\mathrm{AL}$ & Kieser et al. (1993) \\
\hline $\mathrm{C}_{3} \mathrm{H}_{6}$ & $18 \ldots 169 \mathrm{pmol} / \mathrm{mol}$ & Mar-May 1995 & $\mathrm{PF}$ & Herring et al. (1997) \\
\hline 1-butene & mean: $34 \mathrm{pmol} / \mathrm{mol}$ & Apr 1989 & $\mathrm{AL}$ & Kieser et al. (1993) \\
\hline 1-butene & mean: $30 \mathrm{pmol} / \mathrm{mol}$ & May 1989 & $\mathrm{AL}$ & Kieser et al. (1993) \\
\hline cis-2-butene & & $1983-1986$ & NA & Hov et al. (1989) \\
\hline trans-2-butene & & $1983-1986$ & NA & Hov et al. (1989) \\
\hline isobutene & & 1983-1986 & $\mathrm{NA}$ & Hov et al. (1989) \\
\hline isobutene & mean: $77 \mathrm{pmol} / \mathrm{mol}$ & Apr 1989 & $\mathrm{AL}$ & Kieser et al. (1993) \\
\hline isobutene & mean: $115 \mathrm{pmol} / \mathrm{mol}$ & May 1989 & $\mathrm{AL}$ & Kieser et al. (1993) \\
\hline $\mathrm{C}_{2} \mathrm{H}_{2}$ & & 1982,1983 & $\mathrm{NO}$ & Hov et al. (1984) \\
\hline $\mathrm{C}_{2} \mathrm{H}_{2}$ & mean: $11 \mathrm{pmol} / \mathrm{mol}$ & $1982-1985$ & $\mathrm{~nm}$ & Rudolph et al. (1989) \\
\hline $\mathrm{C}_{2} \mathrm{H}_{2}$ & & $1983-1986$ & NA & Hov et al. (1989) \\
\hline $\mathrm{C}_{2} \mathrm{H}_{2}$ & & 1989-1994 & NA & Solberg et al. (1996a) \\
\hline $\mathrm{C}_{2} \mathrm{H}_{2}$ & $900 \ldots 1800 \mathrm{pmol} / \mathrm{mol}$ & Mar 1989 & $\mathrm{BA}$ & Doskey and Gaffney (1992) \\
\hline $\mathrm{C}_{2} \mathrm{H}_{2}$ & mean: $53 \mathrm{pmol} / \mathrm{mol}$ & Apr 1989 & $\mathrm{AL}$ & Kieser et al. (1993) \\
\hline $\mathrm{C}_{2} \mathrm{H}_{2}$ & mean: $80 \mathrm{pmol} / \mathrm{mol}$ & May 1989 & $\mathrm{AL}$ & Kieser et al. (1993) \\
\hline $\mathrm{C}_{2} \mathrm{H}_{2}$ & & 1992-1999 & NA & Albrecht et al. (2002) \\
\hline $\mathrm{C}_{2} \mathrm{H}_{2}$ & $0 \ldots 1600 \mathrm{pmol} / \mathrm{mol}$ & win/spr 1992 & $\mathrm{AL}$ & Jobson et al. (1994) \\
\hline $\mathrm{C}_{2} \mathrm{H}_{2}$ & & Mar-Jun 1993 & NA & Solberg et al. (1996b) \\
\hline $\mathrm{C}_{2} \mathrm{H}_{2}$ & $\begin{array}{ll}\text { bgr.: } & 123.0 \ldots 939.0 \\
\mathrm{pmol} / \mathrm{mol} & \end{array}$ & Feb-May 1994 & NA & Beine et al. (1997b) \\
\hline $\mathrm{C}_{2} \mathrm{H}_{2}$ & & Apr 1994 & $\mathrm{AL}, \mathrm{NW}$ & Ariya et al. (1998) \\
\hline $\mathrm{C}_{2} \mathrm{H}_{2}$ & & Feb-Apr 1995 & $\mathrm{AL}$ & Ariya et al. (1999) \\
\hline $\mathrm{C}_{2} \mathrm{H}_{2}$ & $190 \ldots 1080 \mathrm{pmol} / \mathrm{mol}$ & Mar-May 1995 & $\mathrm{PF}$ & Herring et al. (1997) \\
\hline $\mathrm{C}_{2} \mathrm{H}_{2}$ & mean: $329 \mathrm{pmol} / \mathrm{mol}$ & Mar-May 1996 & NA & Ramacher et al. (1999) \\
\hline $\mathrm{C}_{2} \mathrm{H}_{2}$ & & $1997-1998$ & $\mathrm{SU}$ & Swanson et al. (2003) \\
\hline $\mathrm{C}_{2} \mathrm{H}_{2}$ & & Apr-May 1998 & $\mathrm{AL}$ & Boudries and Bottenheim (2000) \\
\hline $\mathrm{C}_{2} \mathrm{H}_{2}$ & & 1998,2000 & $\mathrm{AL}$ & Bottenheim et al. (2002a) \\
\hline $\mathrm{C}_{2} \mathrm{H}_{2}$ & & Feb-May 2000 & $\mathrm{ARC}$ & $\begin{array}{l}\text { Ridley et al. (2003); Evans et al. } \\
(2003)\end{array}$ \\
\hline $\mathrm{C}_{2} \mathrm{H}_{2}$ & & Feb-May 2000 & $\mathrm{ARC}$ & Blake et al. (2003) \\
\hline $\mathrm{C}_{2} \mathrm{H}_{2}$ & mean: $19 \mathrm{pmol} / \mathrm{mol}$ & $2004-2005$ & ha & Read et al. (2006) \\
\hline $\mathrm{C}_{3} \mathrm{H}_{4}$ & & $1983-1986$ & NA & Hov et al. (1989) \\
\hline isoprene & $0.6 \ldots 7.3 \mathrm{pmol} / \mathrm{mol}$ & Jan 1992 & $\mathrm{AL}$ & Yokouchi et al. (1994) \\
\hline isoprene & $0.6 \ldots 10.3 \mathrm{pmol} / \mathrm{mol}$ & Apr 1992 & $\mathrm{AL}$ & Yokouchi et al. (1994) \\
\hline benzene & & 1982,1983 & $\mathrm{NO}$ & Hov et al. (1984) \\
\hline benzene & $390 \ldots 470 \mathrm{pmol} / \mathrm{mol}$ & Mar 1989 & $\mathrm{BA}$ & Doskey and Gaffney (1992) \\
\hline
\end{tabular}




\begin{tabular}{|c|c|c|c|c|}
\hline species & value & date & site & reference \\
\hline benzene & $200 \ldots 400 \mathrm{pmol} / \mathrm{mol}$ & win/spr 1992 & $\mathrm{AL}$ & Jobson et al. (1994) \\
\hline benzene & & Mar-Jun 1993 & NA & Solberg et al. (1996b) \\
\hline benzene & & Apr 1994 & $\mathrm{AL}, \mathrm{NW}$ & Ariya et al. (1998) \\
\hline benzene & & Feb-Apr 1995 & $\mathrm{AL}$ & Ariya et al. (1999) \\
\hline benzene & & Feb-May 2000 & $\mathrm{AL}$ & Boudries et al. (2002) \\
\hline benzene & & Feb-May 2000 & $\mathrm{ARC}$ & Blake et al. (2003) \\
\hline toluene & & 1982,1983 & $\mathrm{NO}$ & Hov et al. (1984) \\
\hline toluene & $23 \ldots 150 \mathrm{pmol} / \mathrm{mol}$ & Mar 1989 & $\mathrm{BA}$ & Doskey and Gaffney (1992) \\
\hline toluene & & Apr 1994 & $\mathrm{AL}, \mathrm{NW}$ & Ariya et al. (1998) \\
\hline toluene & & Feb-Apr 1995 & $\mathrm{AL}$ & Ariya et al. (1999) \\
\hline total NMHC & $5.6 \ldots 15.7 \mathrm{nmolC} / \mathrm{mol}$ & Mar-May 1993 & $\mathrm{PF}$ & Beine et al. (1996b) \\
\hline \multicolumn{5}{|c|}{ Organic: $\mathrm{C}, \mathrm{H}, \mathrm{O}$} \\
\hline $\mathrm{CH}_{3} \mathrm{OH}$ & & Feb-May 2000 & $\mathrm{AL}$ & Boudries et al. (2002) \\
\hline $\mathrm{C}_{2} \mathrm{H}_{5} \mathrm{OH}$ & & Feb-May 2000 & $\mathrm{AL}$ & Boudries et al. (2002) \\
\hline $\mathrm{HCHO}$ & $\leq 39 \mathrm{pmol} / \mathrm{mol}$ & spr 1988 & AL & Bottenheim et al. (1990) \\
\hline $\mathrm{HCHO}$ & & $1989-1994$ & NA & Solberg et al. (1996a) \\
\hline $\mathrm{HCHO}$ & $100 \ldots 700 \mathrm{pmol} / \mathrm{mol}$ & win 1992 & $\mathrm{AL}$ & de Serves (1994) \\
\hline $\mathrm{HCHO}$ & $30 \ldots 600 \mathrm{pmol} / \mathrm{mol}$ & spr 1992 & $\mathrm{AL}$ & de Serves (1994) \\
\hline $\mathrm{HCHO}$ & & $1992-1999$ & NA & Albrecht et al. (2002) \\
\hline $\mathrm{HCHO}$ & $0.2 \ldots 0.3 \mathrm{nmol} / \mathrm{mol}$ & 1993-1994 & ant & Fuhrer et al. (1996) \\
\hline $\mathrm{HCHO}$ & mean: $0.3 \mathrm{nmol} / \mathrm{mol}$ & 1993-1994 & SU & Fuhrer et al. (1996) \\
\hline $\mathrm{HCHO}$ & mean: $193 \mathrm{pmol} / \mathrm{mol}$ & Apr 1994 & $\mathrm{AL}$ & Shepson et al. (1996) \\
\hline $\mathrm{HCHO}$ & mean: $0.23 \mathrm{nmol} / \mathrm{mol}$ & Jun 1996 & SU & Hutterli et al. (1999) \\
\hline $\mathrm{HCHO}$ & $0.03 \ldots 0.7 \mathrm{nmol} / \mathrm{mol}$ & 1997-1999 & $\mathrm{nm}$ & Riedel et al. (1999) \\
\hline $\mathrm{HCHO}$ & $78 \ldots 372 \mathrm{pmol} / \mathrm{mol}$ & Feb 1998 & AL & Sumner and Shepson (1999) \\
\hline $\mathrm{HCHO}$ & $52 \ldots 690 \mathrm{pmol} / \mathrm{mol}$ & Apr 1998 & $\mathrm{AL}$ & Sumner and Shepson (1999) \\
\hline $\mathrm{HCHO}$ & & 1998,2000 & $\mathrm{AL}$ & Sumner et al. (2002) \\
\hline $\mathrm{HCHO}$ & $0.74 \mathrm{nmol} / \mathrm{mol}$ & sum 1999 & SU & Yang et al. (2002) \\
\hline $\mathrm{HCHO}$ & mean: $103 \mathrm{pmol} / \mathrm{mol}$ & Dec 2000 & $\mathrm{sp}$ & Hutterli et al. (2004) \\
\hline $\mathrm{HCHO}$ & $166 \mathrm{pmol} / \mathrm{mol}$ & 2000 & $\mathrm{AL}$ & Grannas et al. (2002) \\
\hline $\mathrm{HCHO}$ & & Feb-May 2000 & $\mathrm{ARC}$ & $\begin{array}{l}\text { Ridley et al. (2003); Evans et al. } \\
(2003)\end{array}$ \\
\hline $\mathrm{HCHO}$ & $30 \ldots 420 \mathrm{pmol} / \mathrm{mol}$ & Jun-Jul 2000 & $\mathrm{SU}$ & Jacobi et al. (2002) \\
\hline $\mathrm{HCHO}$ & mean: $121 \mathrm{pmol} / \mathrm{mol}$ & Dec 2002 & by & Frey et al. (2005) \\
\hline $\mathrm{HCHO}$ & mean: $154 \mathrm{pmol} / \mathrm{mol}$ & Dec 2002 & ant & Frey et al. (2005) \\
\hline $\mathrm{CH}_{3} \mathrm{CHO}$ & $65 \mathrm{pmol} / \mathrm{mol}$ & spr 1988 & $\mathrm{AL}$ & Bottenheim et al. (1990) \\
\hline $\mathrm{CH}_{3} \mathrm{CHO}$ & & $1989-1994$ & NA & Solberg et al. (1996a) \\
\hline $\mathrm{CH}_{3} \mathrm{CHO}$ & mean: $93 \mathrm{pmol} / \mathrm{mol}$ & Apr 1994 & $\mathrm{AL}$ & Shepson et al. (1996) \\
\hline $\mathrm{CH}_{3} \mathrm{CHO}$ & $53 \mathrm{pmol} / \mathrm{mol}$ & 2000 & $\mathrm{AL}$ & Grannas et al. (2002) \\
\hline $\mathrm{CH}_{3} \mathrm{CHO}$ & & Feb-May 2000 & $\mathrm{AL}$ & Boudries et al. (2002) \\
\hline $\mathrm{CH}_{3} \mathrm{CHO}$ & & Mar-May 2000 & $\mathrm{AL}$ & Guimbaud et al. (2002) \\
\hline $\mathrm{C}_{2} \mathrm{H}_{5} \mathrm{CHO}$ & & Feb-May 2000 & $\mathrm{AL}$ & Boudries et al. (2002) \\
\hline $\mathrm{C}_{3} \mathrm{H}_{7} \mathrm{CHO}$ & & Feb-May 2000 & $\mathrm{AL}$ & Boudries et al. (2002) \\
\hline $\mathrm{CH}_{3} \mathrm{COCH}_{3}$ & $393 \mathrm{pmol} / \mathrm{mol}$ & spr 1988 & $\mathrm{AL}$ & Bottenheim et al. (1990) \\
\hline $\mathrm{CH}_{3} \mathrm{COCH}_{3}$ & & $1989-1994$ & NA & Solberg et al. (1996a) \\
\hline $\mathrm{CH}_{3} \mathrm{COCH}_{3}$ & $901 \ldots 1585 \mathrm{pmol} / \mathrm{mol}$ & Apr 1992 & $\mathrm{AL}$ & Yokouchi et al. (1994) \\
\hline $\mathrm{CH}_{3} \mathrm{COCH}_{3}$ & mean: $1730 \mathrm{pmol} / \mathrm{mol}$ & Apr 1994 & $\mathrm{AL}$ & Shepson et al. (1996) \\
\hline $\mathrm{CH}_{3} \mathrm{COCH}_{3}$ & $385 \mathrm{pmol} / \mathrm{mol}$ & 2000 & $\mathrm{AL}$ & Grannas et al. (2002) \\
\hline $\mathrm{CH}_{3} \mathrm{COCH}_{3}$ & & Feb-May 2000 & $\mathrm{AL}$ & Boudries et al. (2002) \\
\hline $\mathrm{CH}_{3} \mathrm{COCH}_{3}$ & & Mar-May 2000 & $\mathrm{AL}$ & Guimbaud et al. (2002) \\
\hline $\mathrm{CH}_{3} \mathrm{COCH}_{3}$ & & Mar-Apr 2005 & $\mathrm{BA}$ & Tackett et al. (2006) \\
\hline $\mathrm{C}_{2} \mathrm{H}_{5} \mathrm{COCH}_{3}$ & & Feb-May 2000 & $\mathrm{AL}$ & Boudries et al. (2002) \\
\hline $\mathrm{C}_{2} \mathrm{H}_{5} \mathrm{COCH}_{3}$ & & Mar-Apr 2005 & $\mathrm{BA}$ & Tackett et al. (2006) \\
\hline $\mathrm{HCOOH}$ & & 1994-1995 & SU & Dibb et al. (1998) \\
\hline $\mathrm{HCOOH}$ & & Jun 2000 & $\mathrm{SU}$ & Jacobi et al. (2004) \\
\hline $\mathrm{HCOOH}$ & mean: $159 \mathrm{pmol} / \mathrm{mol}$ & Dec 2000 & $\mathrm{SU}$ & Dibb and Arsenault (2002) \\
\hline $\mathrm{HCOOH}$ & mean: $460 \mathrm{pmol} / \mathrm{mol}$ & Jun-Jul 2000 & $\mathrm{SU}$ & Dibb and Arsenault (2002) \\
\hline $\mathrm{HCOOH}$ & mean: $49 \mathrm{nmol} / \mathrm{m}^{3}(\mathrm{STP})$ & Jun-Jul 1993 & SU & Dibb et al. (1994) \\
\hline $\mathrm{CH}_{3} \mathrm{COOH}$ & & 1994-1995 & SU & Dibb et al. (1998) \\
\hline
\end{tabular}




\begin{tabular}{|c|c|c|c|c|}
\hline species & value & date & site & reference \\
\hline $\mathrm{CH}_{3} \mathrm{COOH}$ & & Jun 2000 & SU & Jacobi et al. (2004) \\
\hline $\mathrm{CH}_{3} \mathrm{COOH}$ & mean: $310 \mathrm{pmol} / \mathrm{mol}$ & Dec 2000 & $\mathrm{sp}$ & Dibb and Arsenault (2002) \\
\hline $\mathrm{CH}_{3} \mathrm{COOH}$ & mean: $32 \mathrm{nmol} / \mathrm{m}^{3}(\mathrm{STP})$ & Jun-Jul 1993 & SU & Dibb et al. (1994) \\
\hline $\mathrm{CH}_{3} \mathrm{COOH}$ & mean: $445 \mathrm{pmol} / \mathrm{mol}$ & Jun-Jul 2000 & $\mathrm{SU}$ & Dibb and Arsenault (2002) \\
\hline $\mathrm{CH}_{3} \mathrm{OOH}$ & $<$ DL...0.89 nmol $/ \mathrm{mol}$ & $1997-1999$ & $\mathrm{~nm}$ & Riedel et al. (2000) \\
\hline $\mathrm{CH}_{3} \mathrm{OOH}$ & & Feb-May 2000 & $\mathrm{ARC}$ & $\begin{array}{l}\text { Ridley et al. (2003); Evans et al. } \\
(2003)\end{array}$ \\
\hline $\mathrm{CH}_{3} \mathrm{OOH}$ & mean: $317 \mathrm{pmol} / \mathrm{mol}$ & Dec 2001 & ant & Frey et al. (2005) \\
\hline $\mathrm{CH}_{3} \mathrm{OOH}$ & mean: $426 \mathrm{pmol} / \mathrm{mol}$ & Dec 2002 & by & Frey et al. (2005) \\
\hline $\mathrm{CH}_{3} \mathrm{OOH}$ & mean: $102 \mathrm{pmol} / \mathrm{mol}$ & Jan 2003 & $\mathrm{sp}$ & Frey et al. (2005) \\
\hline $\mathrm{ROOH}+\mathrm{H}_{2} \mathrm{O}_{2}$ & $10 \ldots 40 \mathrm{pmol} / \mathrm{mol}$ & win 1992 & $\mathrm{AL}$ & Yokouchi et al. (1994) \\
\hline $\mathrm{ROOH}+\mathrm{H}_{2} \mathrm{O}_{2}$ & $100 \ldots 400 \mathrm{pmol} / \mathrm{mol}$ & spr 1992 & $\mathrm{AL}$ & Yokouchi et al. (1994) \\
\hline $\mathrm{RO}_{2}$ & & Feb-May 2000 & $\mathrm{ARC}$ & $\begin{array}{l}\text { Ridley et al. (2003); Evans et al. } \\
(2003)\end{array}$ \\
\hline $\mathrm{RO}_{2}+\mathrm{HO}_{2}$ & & 2000 & $\mathrm{sp}$ & Mauldin III et al. (2004) \\
\hline $\mathrm{CO}$ & & $1980-1982$ & $\mathrm{BA}$ & Khalil and Rasmussen (1983) \\
\hline $\mathrm{CO}$ & mean: $131 \mathrm{nmol} / \mathrm{mol}$ & $1988-1989$ & DY & Davidson et al. (1993b,a) \\
\hline $\mathrm{CO}$ & $52.3 \ldots 302.0 \mathrm{nmol} / \mathrm{mol}$ & $1992-1993$ & NA & Beine (1999) \\
\hline $\mathrm{CO}$ & $89 . \ldots 216 \mathrm{nmol} / \mathrm{mol}$ & Mar-May 1995 & $\mathrm{PF}$ & Herring et al. (1997) \\
\hline $\mathrm{CO}$ & $90 \mathrm{nmol} / \mathrm{mol}$ & sum 1999 & $\mathrm{SU}$ & Yang et al. (2002) \\
\hline $\mathrm{CO}$ & $110 \mathrm{nmol} / \mathrm{mol}$ & sum 2000 & $\mathrm{SU}$ & Yang et al. (2002) \\
\hline $\mathrm{CO}$ & & Feb-Mar 2000 & $\mathrm{AL}$ & Bottenheim et al. (2002b) \\
\hline $\mathrm{CO}$ & & Feb-May 2000 & $\mathrm{ARC}$ & $\begin{array}{l}\text { Ridley et al. (2003); Evans et al. } \\
(2003)\end{array}$ \\
\hline
\end{tabular}

Organic: $\mathrm{C}, \mathrm{H}, \mathrm{O}, \mathrm{N}$

\begin{tabular}{|c|c|c|c|c|}
\hline PAN & $189 \ldots 234 \mathrm{pmol} / \mathrm{mol}$ & Mar 1985 & $\mathrm{AL}$ & Bottenheim et al. (1986) \\
\hline PAN & $200 \ldots 500 \mathrm{pmol} / \mathrm{mol}$ & spr 1988 & $\mathrm{AL}$ & Barrie and Delmas (1994) \\
\hline PAN & & Mar-Apr 1988 & $\mathrm{AL}$ & Bottenheim et al. (1993) \\
\hline PAN & $150 \ldots 600 \mathrm{pmol} / \mathrm{mol}$ & win/spr 1992 & $\mathrm{AL}$ & Barrie et al. (1994a) \\
\hline PAN & $27 \ldots 371 \mathrm{pmol} / \mathrm{mol}$ & Mar-May 1993 & $\mathrm{PF}$ & Beine et al. (1996b) \\
\hline PAN & & Mar-Jun 1994 & $\mathrm{ARC}$ & Jaeschke et al. (1997) \\
\hline PAN & $69.0 \ldots 729.0 \mathrm{pmol} / \mathrm{mol}$ & Feb-May 1994 & NA & Beine et al. (1997a) \\
\hline PAN & $19.7 \ldots 1608.2 \mathrm{pmol} / \mathrm{mol}$ & 1994-1996 & NA & Beine and Krognes (2000) \\
\hline PAN & $\begin{array}{lll}\text { bgr.: } & 2.9 & \ldots 739.0 \\
\mathrm{pmol} / \mathrm{mol} & & \end{array}$ & Mar-May 1995 & $\mathrm{PF}$ & Beine et al. (1997a) \\
\hline PAN & $100 \ldots 420 \mathrm{pmol} / \mathrm{mol}$ & Mar 1998 & NA & Jacobi et al. (1999) \\
\hline PAN & & 1998-1999 & SU & Ford et al. (2002) \\
\hline PAN & & 1998-1999 & $\mathrm{SU}$ & Dassau et al. (2004) \\
\hline PAN & & 1998,2000 & $\mathrm{AL}$ & Dassau et al. (2004) \\
\hline PAN & mean: $13 \mathrm{pmol} / \mathrm{mol}$ & Jan-Feb 1999 & $\mathrm{~nm}$ & Jacobi et al. (2000) \\
\hline PAN & mean: $18 \mathrm{pmol} / \mathrm{mol}$ & Mar 1999 & ant & Jacobi and Schrems (1999) \\
\hline PAN & & Feb-May 2000 & $\mathrm{ARC}$ & $\begin{array}{l}\text { Ridley et al. (2003); Evans et al. } \\
(2003)\end{array}$ \\
\hline $\mathrm{PPN}$ & mean: $26 \mathrm{pmol} / \mathrm{mol}$ & Feb-May 1994 & NA & Solberg et al. (1997a) \\
\hline methyl nitrate & mean: $10 \mathrm{pmol} / \mathrm{mol}$ & Jan-Mar 1997 & $\mathrm{~nm}$ & $\begin{array}{l}\text { Jones et al. (1999); Weller et al. } \\
(2002)\end{array}$ \\
\hline methyl nitrate & & 1997-1998 & $\mathrm{SU}$ & Swanson et al. (2003) \\
\hline methyl nitrate & mean: $9.5 \mathrm{pmol} / \mathrm{mol}$ & Feb 1999 & $\mathrm{~nm}$ & Weller et al. (2002) \\
\hline methyl nitrate & mean: $84 \mathrm{pmol} / \mathrm{mol}$ & Feb 1999 & $\mathrm{~nm}$ & Fischer et al. (2002) \\
\hline methyl nitrate & & 2000 & $\mathrm{sp}$ & Swanson et al. (2004) \\
\hline methyl nitrate & & Feb-May 2000 & $\mathrm{ARC}$ & Blake et al. (2003) \\
\hline ethyl nitrate & mean: $3 \mathrm{pmol} / \mathrm{mol}$ & Jan-Mar 1997 & $\mathrm{~nm}$ & $\begin{array}{l}\text { Jones et al. (1999); Weller et al. } \\
(2002)\end{array}$ \\
\hline ethyl nitrate & & 1997-1998 & SU & Swanson et al. (2003) \\
\hline ethyl nitrate & mean: $2.3 \mathrm{pmol} / \mathrm{mol}$ & Feb 1999 & $\mathrm{~nm}$ & Weller et al. (2002) \\
\hline ethyl nitrate & mean: $4.6 \mathrm{pmol} / \mathrm{mol}$ & Feb 1999 & $\mathrm{~nm}$ & Fischer et al. (2002) \\
\hline ethyl nitrate & & 2000 & $\mathrm{sp}$ & Swanson et al. (2004) \\
\hline ethyl nitrate & & Feb-May 2000 & $\mathrm{ARC}$ & Blake et al. (2003) \\
\hline 1-propyl nitrate & $3.14 \ldots 3.33 \mathrm{pmol} / \mathrm{mol}$ & Jan-Apr 1992 & $\mathrm{AL}$ & Muthuramu et al. (1994) \\
\hline
\end{tabular}




\begin{tabular}{|c|c|c|c|c|}
\hline species & value & date & site & reference \\
\hline 1-propyl nitrate & & $1997-1998$ & SU & Swanson et al. (2003) \\
\hline 1-propyl nitrate & mean: $1.1 \mathrm{pmol} / \mathrm{mol}$ & Feb 1999 & $\mathrm{~nm}$ & Weller et al. (2002) \\
\hline 1-propyl nitrate & mean: $1.1 \mathrm{pmol} / \mathrm{mol}$ & Feb 1999 & $\mathrm{~nm}$ & Fischer et al. (2002) \\
\hline 2-propyl nitrate & $12.44 \ldots 13.08 \mathrm{pmol} / \mathrm{mol}$ & Jan-Apr 1992 & $\mathrm{AL}$ & Muthuramu et al. (1994) \\
\hline 2-propyl nitrate & & $1997-1998$ & $\mathrm{SU}$ & Swanson et al. (2003) \\
\hline 2-propyl nitrate & mean: $1.2 \mathrm{pmol} / \mathrm{mol}$ & Feb 1999 & $\mathrm{~nm}$ & Weller et al. (2002) \\
\hline 2-propyl nitrate & mean: $0.7 \mathrm{pmol} / \mathrm{mol}$ & Feb 1999 & $\mathrm{~nm}$ & Fischer et al. (2002) \\
\hline 2-propyl nitrate & & 2000 & $\mathrm{sp}$ & Swanson et al. (2004) \\
\hline 2-propyl nitrate & & Feb-May 2000 & ARC & Blake et al. (2003) \\
\hline 1-butyl nitrate & $1.18 \ldots 1.7 \mathrm{pmol} / \mathrm{mol}$ & Jan-Apr 1992 & $\mathrm{AL}$ & Muthuramu et al. (1994) \\
\hline 1-butyl nitrate & mean: $0.03 \mathrm{pmol} / \mathrm{mol}$ & Feb 1999 & $\mathrm{~nm}$ & Fischer et al. (2002) \\
\hline 2-butyl nitrate & $13.73 \ldots 18.41 \mathrm{pmol} / \mathrm{mol}$ & Jan-Apr 1992 & $\overline{\mathrm{AL}}$ & Muthuramu et al. (1994) \\
\hline 2-butyl nitrate & & $1997-1998$ & $\mathrm{SU}$ & Swanson et al. (2003) \\
\hline 2-butyl nitrate & mean: $0.5 \mathrm{pmol} / \mathrm{mol}$ & Feb 1999 & $\mathrm{~nm}$ & Fischer et al. (2002) \\
\hline 2-butyl nitrate & & Feb-May 2000 & $\mathrm{ARC}$ & Blake et al. (2003) \\
\hline 1-pentyl nitrate & $0.53 \ldots 1.01 \mathrm{pmol} / \mathrm{mol}$ & Jan-Apr 1992 & $\mathrm{AL}$ & Muthuramu et al. (1994) \\
\hline 1-pentyl nitrate & mean: $0.7 \mathrm{pmol} / \mathrm{mol}$ & Feb 1999 & $\mathrm{~nm}$ & Fischer et al. (2002) \\
\hline 2-pentyl nitrate & $2.47 \ldots 5.44 \mathrm{pmol} / \mathrm{mol}$ & Jan-Apr 1992 & $\mathrm{AL}$ & Muthuramu et al. (1994) \\
\hline 2-pentyl nitrate & mean: < DL & Feb 1999 & $\mathrm{~nm}$ & Fischer et al. (2002) \\
\hline 3-pentyl nitrate & $2.31 \ldots 4.31 \mathrm{pmol} / \mathrm{mol}$ & Jan-Apr 1992 & $\mathrm{AL}$ & Muthuramu et al. (1994) \\
\hline 3-pentyl nitrate & mean: $0.03 \mathrm{pmol} / \mathrm{mol}$ & Feb 1999 & $\mathrm{~nm}$ & Fischer et al. (2002) \\
\hline $\begin{array}{l}\text { 2-methyl-1-butyl ni- } \\
\text { trate }\end{array}$ & $0.39 \ldots 0.77 \mathrm{pmol} / \mathrm{mol}$ & Jan-Apr 1992 & $\mathrm{AL}$ & Muthuramu et al. (1994) \\
\hline $\begin{array}{l}\text { 3-methyl-1-butyl ni- } \\
\text { trate }\end{array}$ & $0.30 \ldots 0.55 \mathrm{pmol} / \mathrm{mol}$ & Jan-Apr 1992 & $\mathrm{AL}$ & Muthuramu et al. (1994) \\
\hline $\begin{array}{l}\text { 3-methyl-2-butyl ni- } \\
\text { trate }\end{array}$ & $2.32 \ldots 4.84 \mathrm{pmol} / \mathrm{mol}$ & Jan-Apr 1992 & $\mathrm{AL}$ & Muthuramu et al. (1994) \\
\hline 1-hexyl nitrate & mean: $0.5 \mathrm{pmol} / \mathrm{mol}$ & Feb 1999 & $\mathrm{~nm}$ & Fischer et al. (2002) \\
\hline 2-hexyl nitrate & $0.98 \ldots 2.46 \mathrm{pmol} / \mathrm{mol}$ & Jan-Apr 1992 & $\mathrm{AL}$ & Muthuramu et al. (1994) \\
\hline 2-hexyl nitrate & mean: $1.0 \mathrm{pmol} / \mathrm{mol}$ & Feb 1999 & $\mathrm{~nm}$ & Fischer et al. (2002) \\
\hline 3-hexyl nitrate & $1.65 \ldots 4.27 \mathrm{pmol} / \mathrm{mol}$ & Jan-Apr 1992 & $\mathrm{AL}$ & Muthuramu et al. (1994) \\
\hline 3-hexyl nitrate & mean: $0.08 \mathrm{pmol} / \mathrm{mol}$ & Feb 1999 & $\mathrm{~nm}$ & Fischer et al. (2002) \\
\hline 2-heptyl nitrate & $0.56 \ldots 1.45 \mathrm{pmol} / \mathrm{mol}$ & Jan-Apr 1992 & $\mathrm{AL}$ & Muthuramu et al. (1994) \\
\hline 2-heptyl nitrate & mean: $0.18 \mathrm{pmol} / \mathrm{mol}$ & Feb 1999 & $\mathrm{~nm}$ & Fischer et al. (2002) \\
\hline 3-heptyl nitrate & $0.68 \ldots 1.86 \mathrm{pmol} / \mathrm{mol}$ & Jan-Apr 1992 & $\mathrm{AL}$ & Muthuramu et al. (1994) \\
\hline 4-heptyl nitrate & mean: $0.02 \mathrm{pmol} / \mathrm{mol}$ & Feb 1999 & $\mathrm{~nm}$ & Fischer et al. (2002) \\
\hline alkyl nitrates & $11 \ldots 66 \mathrm{pmol} / \mathrm{mol}$ & Mar-May 1993 & $\mathrm{PF}$ & Beine et al. (1996b) \\
\hline organic nitrates & & Mar-Apr 1988 & $\mathrm{AL}$ & Bottenheim et al. (1993) \\
\hline organic nitrates & $0 . \ldots 2828.0 \mathrm{pmol} / \mathrm{mol}$ & 1997-1999 (spr) & NA & Beine et al. (2001) \\
\hline organic nitrates & mean: $1038.8 \mathrm{ng} / \mathrm{m}^{3}$ & Apr-May 2000 & $\mathrm{AL}$ & Ianniello et al. (2002) \\
\hline organic nitrates & mean: $309 \mathrm{ng} / \mathrm{m}^{3}$ & Feb 2000 & $\mathrm{AL}$ & Ianniello et al. (2002) \\
\hline hydroxy nitrates & mean: $1.1 \mathrm{pmol} / \mathrm{mol}$ & Feb 1999 & $\mathrm{~nm}$ & Fischer et al. (2002) \\
\hline organic dinitrates & mean: $5.9 \mathrm{pmol} / \mathrm{mol}$ & Feb 1999 & $\mathrm{~nm}$ & Fischer et al. (2002) \\
\hline
\end{tabular}

\section{Chlorine (inorganic)}

\begin{tabular}{|c|c|c|c|c|}
\hline inorg-Cl & $0 \ldots 500 \mathrm{pmol} / \mathrm{mol}$ & win/spr 1992 & $\mathrm{AL}$ & Barrie et al. (1994a) \\
\hline photolyzable $\mathrm{Cl}$ & $<9 \ldots 100 \mathrm{pmol} / \mathrm{mol}$ & Feb-Apr 1995 & $\mathrm{AL}$ & Impey et al. (1997) \\
\hline photolyzable $\mathrm{Cl}$ & & Mar-Apr 1997 & $\mathrm{AL}$ & Impey et al. (1999) \\
\hline gaseous $\mathrm{Cl}$ & & 1996-1999 & NA & Hara et al. (2002a) \\
\hline gaseous $\mathrm{Cl}$ & & Mar-Apr 2000 & NA & Hara et al. (2002b) \\
\hline $\mathrm{HCl}$ & $0 \ldots 303.6 \mathrm{pmol} / \mathrm{mol}$ & 1997-1999 (spr) & NA & Beine et al. (2001) \\
\hline $\mathrm{HCl}$ & mean: $11.3 \mathrm{ng} / \mathrm{m}^{3}$ & Feb 2000 & $\mathrm{AL}$ & Ianniello et al. (2002) \\
\hline $\mathrm{HCl}$ & mean: $32.7 \mathrm{ng} / \mathrm{m}^{3}$ & Apr-May 2000 & $\mathrm{AL}$ & Ianniello et al. (2002) \\
\hline $\mathrm{HCl}$ & & $2000-2001$ & $\mathrm{du}$ & Jourdain and Legrand (2002) \\
\hline $\mathrm{Cl}$ atoms & $3.9 \times 10^{3} \ldots 7.7 \times 10^{4} \mathrm{~cm}^{-3}$ & spr 1992 & $\mathrm{AL}$ & Jobson et al. (1994) \\
\hline $\mathrm{Cl}$ atoms & $3.8 \times 10^{3} \ldots 1.0 \times 10^{4} \mathrm{~cm}^{-3}$ & spr 1992 & $\mathrm{AL}$ & Muthuramu et al. (1994) \\
\hline $\mathrm{Cl}$ atoms & $4.5 \times 10^{3} \mathrm{~cm}^{-3}$ & spr 1994 & NW & Ariya et al. (1998) \\
\hline $\mathrm{Cl}$ atoms & $7.5 \times 10^{4} \mathrm{~cm}^{-3}$ & Apr-May 1998 & $\mathrm{AL}$ & Boudries and Bottenheim (2000) \\
\hline $\mathrm{Cl}$ atoms & $1.7 \times 10^{3} \ldots 3.4 \times 10^{4} \mathrm{~cm}^{-3}$ & 2004-2005 & ha & Read et al. (2006) \\
\hline $\mathrm{ClO}$ & & 1995,1996 & NA & Tuckermann et al. (1997) \\
\hline
\end{tabular}




\begin{tabular}{lllll}
\hline species & value & date & site & reference \\
\hline $\mathrm{OClO}$ & $\mathrm{SCD}$ & 1995 & $\mathrm{~mm}$ & Kreher et al. (1997) \\
$\mathrm{OClO}$ & & Jan-May 1995 & $\mathrm{KA}$ & Miller et al. (1997) \\
\hline $\mathrm{Cl}_{2}$ & $<\mathrm{DL}(2 \mathrm{pmol} / \mathrm{mol})$ & Feb-Mar 2000 & $\mathrm{AL}$ & $\begin{array}{l}\text { Foster et al. (2001); Spicer et al. } \\
(2002)\end{array}$ \\
\hline
\end{tabular}

Chlorine (organic)

\begin{tabular}{|c|c|c|c|c|}
\hline org-Cl & $1200 \ldots 3400 \mathrm{pmol} / \mathrm{mol}$ & win/spr 1992 & $\mathrm{AL}$ & Barrie et al. (1994a) \\
\hline $\mathrm{CH}_{3} \mathrm{Cl}$ & & $1980-1982$ & $\mathrm{BA}$ & Khalil and Rasmussen (1983) \\
\hline $\mathrm{CH}_{3} \mathrm{Cl}$ & & 1982,1983 & $\mathrm{NO}$ & Hov et al. (1984) \\
\hline $\mathrm{CH}_{2} \mathrm{Cl}_{2}$ & & 1982,1983 & $\mathrm{NO}$ & Hov et al. (1984) \\
\hline $\mathrm{CH}_{2} \mathrm{Cl}_{2}$ & $53.4 \ldots 69.8 \mathrm{pmol} / \mathrm{mol}$ & Apr 1992 & $\mathrm{AL}$ & Yokouchi et al. (1994) \\
\hline $\mathrm{CHCl}_{3}$ & & 1982,1983 & $\mathrm{NO}$ & Hov et al. (1984) \\
\hline $\mathrm{CHCl}_{3}$ & mean: $23.0 \mathrm{pmol} / \mathrm{mol}$ & 1988-1989 & DY & Davidson et al. (1993b,a) \\
\hline $\mathrm{CHCl}_{3}$ & $9.7 \ldots 15.8 \mathrm{pmol} / \mathrm{mol}$ & Apr 1992 & $\mathrm{AL}$ & Yokouchi et al. (1994) \\
\hline $\mathrm{CCl}_{4}$ & & 1982,1983 & $\mathrm{NO}$ & Hov et al. (1984) \\
\hline $\mathrm{CCl}_{4}$ & mean: $125 \mathrm{pmol} / \mathrm{mol}$ & 1988-1989 & DY & Davidson et al. (1993b,a) \\
\hline $\mathrm{CCl}_{4}$ & mean: $95 \mathrm{pmol} / \mathrm{mol}$ & Feb 1999 & $\mathrm{~nm}$ & Fischer et al. (2002) \\
\hline $\mathrm{CH}_{3} \mathrm{CCl}_{3}$ & & $1980-1982$ & $\mathrm{BA}$ & Khalil and Rasmussen (1983) \\
\hline $\mathrm{CH}_{3} \mathrm{CCl}_{3}$ & & 1982,1983 & $\mathrm{NO}$ & Hov et al. (1984) \\
\hline $\mathrm{CH}_{3} \mathrm{CCl}_{3}$ & mean: $211 \mathrm{pmol} / \mathrm{mol}$ & 1988-1989 & DY & Davidson et al. (1993b,a) \\
\hline $\mathrm{CH}_{3} \mathrm{CCl}_{3}$ & mean: $75 \mathrm{pmol} / \mathrm{mol}$ & Feb 1999 & $\mathrm{~nm}$ & Fischer et al. (2002) \\
\hline $\mathrm{C}_{2} \mathrm{Cl}_{6}$ & mean: $0.06 \mathrm{pmol} / \mathrm{mol}$ & Feb 1999 & $\mathrm{~nm}$ & Fischer et al. (2002) \\
\hline $\mathrm{C}_{2} \mathrm{HCl}_{3}$ & & $1980-1982$ & $\mathrm{BA}$ & Khalil and Rasmussen (1983) \\
\hline $\mathrm{C}_{2} \mathrm{HCl}_{3}$ & & 1982,1983 & $\mathrm{NO}$ & Hov et al. (1984) \\
\hline $\mathrm{C}_{2} \mathrm{HCl}_{3}$ & $5.4 \ldots 11.5 \mathrm{pmol} / \mathrm{mol}$ & Jan 1992 & $\mathrm{AL}$ & Yokouchi et al. (1994) \\
\hline $\mathrm{C}_{2} \mathrm{HCl}_{3}$ & $0.5 \ldots 4.3 \mathrm{pmol} / \mathrm{mol}$ & Apr 1992 & $\mathrm{AL}$ & Yokouchi et al. (1994) \\
\hline $\mathrm{C}_{2} \mathrm{HCl}_{3}$ & $0.1 \ldots 1.2 \mathrm{pmol} / \mathrm{mol}$ & Apr 1992 & SW & Hopper et al. (1994a) \\
\hline $\mathrm{C}_{2} \mathrm{HCl}_{3}$ & $0.17 \ldots 4.77 \mathrm{pmol} / \mathrm{mol}$ & Apr 1992 & $\mathrm{ARC}$ & Leaitch et al. (1994) \\
\hline $\mathrm{C}_{2} \mathrm{HCl}_{3}$ & mean: < DL & Feb 1999 & $\mathrm{~nm}$ & Fischer et al. (2002) \\
\hline $\mathrm{C}_{2} \mathrm{Cl}_{4}$ & & $1980-1982$ & $\mathrm{BA}$ & Khalil and Rasmussen (1983) \\
\hline $\mathrm{C}_{2} \mathrm{Cl}_{4}$ & & 1982,1983 & $\mathrm{NO}$ & Hov et al. (1984) \\
\hline $\mathrm{C}_{2} \mathrm{Cl}_{4}$ & $6.6 \ldots 9.7 \mathrm{pmol} / \mathrm{mol}$ & Jan 1992 & $\mathrm{AL}$ & Yokouchi et al. (1994) \\
\hline $\mathrm{C}_{2} \mathrm{Cl}_{4}$ & $4.3 \ldots 9.5 \mathrm{pmol} / \mathrm{mol}$ & Apr 1992 & $\mathrm{AL}$ & Yokouchi et al. (1994) \\
\hline $\mathrm{C}_{2} \mathrm{Cl}_{4}$ & $6.08 \ldots 15.05 \mathrm{pmol} / \mathrm{mol}$ & Apr 1992 & $\mathrm{ARC}$ & Leaitch et al. (1994) \\
\hline $\mathrm{C}_{2} \mathrm{Cl}_{4}$ & mean: $0.3 \mathrm{pmol} / \mathrm{mol}$ & Feb 1999 & $\mathrm{~nm}$ & Fischer et al. (2002) \\
\hline chloroacetaldehyde & & Jan-Apr 2005 & $\mathrm{BA}$ & Keil and Shepson (2006) \\
\hline chloroacetone & & Jan-Apr 2005 & $\mathrm{BA}$ & Keil and Shepson (2006) \\
\hline \multicolumn{5}{|c|}{ Chlorine and Fluorine (organic) } \\
\hline $\mathrm{CFCl}_{3}(\mathrm{~F} 11)$ & & $1980-1982$ & $\mathrm{BA}$ & Khalil and Rasmussen (1983) \\
\hline $\mathrm{CFCl}_{3}(\mathrm{~F} 11)$ & & 1982,1983 & $\mathrm{NO}$ & Hov et al. (1984) \\
\hline $\mathrm{CFCl}_{3}(\mathrm{~F} 11)$ & mean: $285 \mathrm{pmol} / \mathrm{mol}$ & 1988-1989 & DY & Davidson et al. (1993b,a) \\
\hline $\mathrm{CF}_{2} \mathrm{Cl}_{2}(\mathrm{~F} 12)$ & & $1980-1982$ & $\mathrm{BA}$ & Khalil and Rasmussen (1983) \\
\hline $\mathrm{CF}_{2} \mathrm{Cl}_{2}(\mathrm{~F} 12)$ & & 1982,1983 & $\mathrm{NO}$ & Hov et al. (1984) \\
\hline $\mathrm{CF}_{2} \mathrm{Cl}_{2}(\mathrm{~F} 12)$ & mean: $465 \mathrm{pmol} / \mathrm{mol}$ & 1988-1989 & DY & Davidson et al. (1993b,a) \\
\hline $\mathrm{C}_{2} \mathrm{~F}_{3} \mathrm{Cl}_{3}(\mathrm{~F} 113)$ & & 1982,1983 & $\mathrm{NO}$ & Hov et al. (1984) \\
\hline $\mathrm{C}_{2} \mathrm{~F}_{3} \mathrm{Cl}_{3}(\mathrm{~F} 113)$ & mean: $44.6 \mathrm{pmol} / \mathrm{mol}$ & 1988-1989 & DY & Davidson et al. (1993b,a) \\
\hline $\mathrm{C}_{2} \mathrm{~F}_{4} \mathrm{Cl}_{2}(\mathrm{~F} 114)$ & & 1982,1983 & $\mathrm{NO}$ & Hov et al. (1984) \\
\hline $\mathrm{CHF}_{2} \mathrm{Cl}$ & & $1980-1982$ & $\mathrm{BA}$ & Khalil and Rasmussen (1983) \\
\hline
\end{tabular}

Bromine (inorganic)

\begin{tabular}{lllll}
\hline gaseous $\mathrm{Br}$ & $7.4 \mathrm{ng} / \mathrm{m}^{3}(\mathrm{STP})$ & Nov-Dec 1970 & $\mathrm{sp}$ & Duce et al. (1973) \\
gaseous $\mathrm{Br}$ & $7.9 \mathrm{ng} / \mathrm{m}^{3}(\mathrm{STP})$ & Nov-Dec 1970 & $\mathrm{~mm}$ & $\begin{array}{l}\text { Duce et al. (1973) } \\
\text { gaseous } \mathrm{Br}\end{array}$ \\
$\begin{array}{l}\text { gas }-\mathrm{Br} \\
\text { inorg}-\mathrm{Br}\end{array}$ & $0.3 \ldots 61 \mathrm{pmol} / \mathrm{mol}$ & $1976-1980$ & $\mathrm{BA}$ & $\begin{array}{l}\text { Berg et al. (1983) } \\
\text { inorg }-\mathrm{Br}\end{array}$ \\
$\begin{array}{l}\text { inorg }-\mathrm{Br} \\
\text { photolyzable } \mathrm{Br}\end{array}$ & $<45 \mathrm{ng} / \mathrm{m}^{3}$ & Mar-Apr 1989 & $\mathrm{BA}$ & Sturges et al. (1993c) \\
gaseous $\mathrm{Br}$ & $<4 \ldots 38 \mathrm{pmol} / \mathrm{mol}$ & Mar-Apr 1990 & $\mathrm{BA}$ & Sturges et al. (1993b) \\
\hline
\end{tabular}




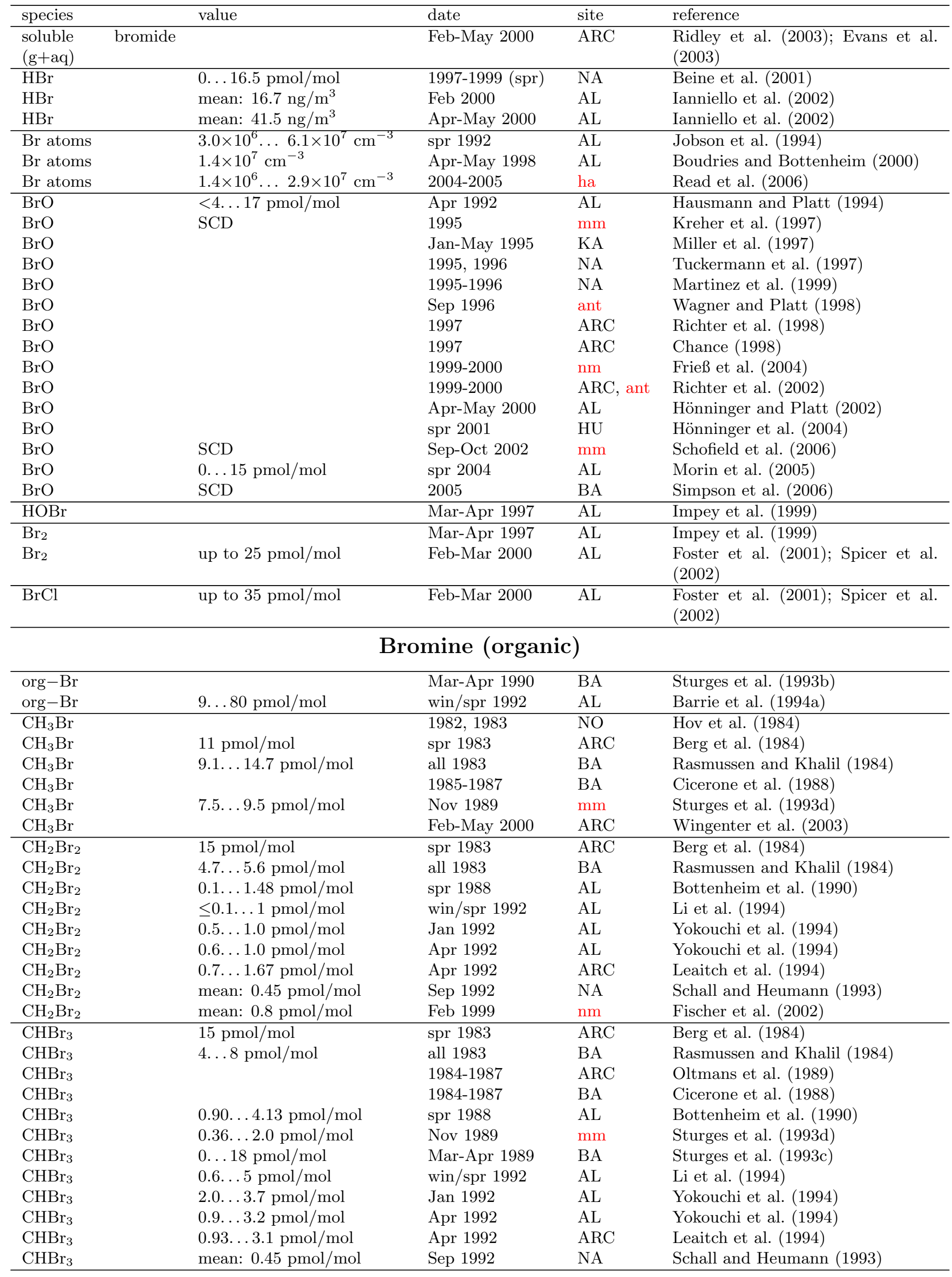




\begin{tabular}{|c|c|c|c|c|}
\hline species & value & date & site & reference \\
\hline $\mathrm{CHBr}_{3}$ & mean: $0.3 \mathrm{pmol} / \mathrm{mol}$ & Feb 1999 & $\mathrm{~nm}$ & Fischer et al. (2002) \\
\hline $\mathrm{CHBr}_{3}$ & & 2000 & $\mathrm{sp}$ & Swanson et al. (2004) \\
\hline $\mathrm{CHBr}_{3}$ & & Mar 2004 & $\mathrm{HU}$ & Carpenter et al. (2005) \\
\hline $\mathrm{C}_{2} \mathrm{H}_{4} \mathrm{Br}_{2}$ & $0.10 \ldots 0.25 \mathrm{pmol} / \mathrm{mol}$ & spr 1988 & $\mathrm{AL}$ & Bottenheim et al. (1990) \\
\hline $\mathrm{CH}_{2} \mathrm{BrCH}_{2} \mathrm{Br}$ & $11 \mathrm{pmol} / \mathrm{mol}$ & spr 1983 & $\mathrm{ARC}$ & Berg et al. (1984) \\
\hline $\mathrm{CH}_{2} \mathrm{BrCH}_{2} \mathrm{Br}$ & $1.0 \ldots 1.9 \mathrm{pmol} / \mathrm{mol}$ & all 1983 & $\mathrm{BA}$ & Rasmussen and Khalil (1984) \\
\hline $\mathrm{CH}_{2} \mathrm{BrCH}_{2} \mathrm{Br}$ & mean: $0.1 \mathrm{pmol} / \mathrm{mol}$ & Feb 1999 & $\mathrm{~nm}$ & Fischer et al. (2002) \\
\hline bromoacetaldehyde & & Jan-Apr 2005 & $\mathrm{BA}$ & Keil and Shepson (2006) \\
\hline bromoacetone & & Jan-Apr 2005 & $\mathrm{BA}$ & Keil and Shepson (2006) \\
\hline $\mathrm{CH}_{2} \mathrm{BrCl}$ & $2.3 \ldots 3.1 \mathrm{pmol} / \mathrm{mol}$ & all 1983 & $\mathrm{BA}$ & Rasmussen and Khalil (1984) \\
\hline $\mathrm{CH}_{2} \mathrm{BrCl}$ & $\leq 0.2 \mathrm{pmol} / \mathrm{mol}$ & win/spr 1992 & $\mathrm{AL}$ & Li et al. (1994) \\
\hline $\mathrm{CH}_{2} \mathrm{BrCl}$ & $0.15 \ldots 0.34 \mathrm{pmol} / \mathrm{mol}$ & Apr 1992 & $\mathrm{AL}$ & Yokouchi et al. (1994) \\
\hline $\mathrm{CHBr}_{2} \mathrm{Cl}$ & $0.17 \ldots 0.53 \mathrm{pmol} / \mathrm{mol}$ & spr 1988 & $\mathrm{AL}$ & Bottenheim et al. (1990) \\
\hline $\mathrm{CHBr}_{2} \mathrm{Cl}$ & $0 \ldots 1.6 \mathrm{pmol} / \mathrm{mol}$ & Mar-Apr 1989 & $\mathrm{BA}$ & Sturges et al. (1993c) \\
\hline $\mathrm{CHBr}_{2} \mathrm{Cl}$ & $0.06 \ldots 0.4 \mathrm{pmol} / \mathrm{mol}$ & win/spr 1992 & $\mathrm{AL}$ & Li et al. (1994) \\
\hline $\mathrm{CHBr}_{2} \mathrm{Cl}$ & $0.1 \ldots 0.5 \mathrm{pmol} / \mathrm{mol}$ & Jan 1992 & $\mathrm{AL}$ & Yokouchi et al. (1994) \\
\hline $\mathrm{CHBr}_{2} \mathrm{Cl}$ & $0.1 \ldots 0.4 \mathrm{pmol} / \mathrm{mol}$ & Apr 1992 & $\mathrm{AL}$ & Yokouchi et al. (1994) \\
\hline $\mathrm{CHBr}_{2} \mathrm{Cl}$ & $0.16 \ldots 0.36 \mathrm{pmol} / \mathrm{mol}$ & Apr 1992 & $\mathrm{ARC}$ & Leaitch et al. (1994) \\
\hline $\mathrm{CHBr}_{2} \mathrm{Cl}$ & mean: $0.33 \mathrm{pmol} / \mathrm{mol}$ & Sep 1992 & NA & Schall and Heumann (1993) \\
\hline $\mathrm{CHBr}_{2} \mathrm{Cl}$ & mean: $0.02 \mathrm{pmol} / \mathrm{mol}$ & Feb 1999 & $\mathrm{~nm}$ & Fischer et al. (2002) \\
\hline $\mathrm{CHBrCl}_{2}$ & $0 \ldots 1.6 \mathrm{pmol} / \mathrm{mol}$ & Mar-Apr 1989 & $\mathrm{BA}$ & Sturges et al. (1993c) \\
\hline $\mathrm{CHBrCl}_{2}$ & $0.11 \ldots 0.39 \mathrm{pmol} / \mathrm{mol}$ & Apr 1992 & $\mathrm{AL}$ & Yokouchi et al. (1994) \\
\hline $\mathrm{CHBrCl}_{2}$ & $0.3 \ldots 1.3 \mathrm{pmol} / \mathrm{mol}$ & win/spr 1992 & $\mathrm{AL}$ & Li et al. (1994) \\
\hline $\mathrm{CHBrCl}_{2}$ & $0.38 \ldots 0.73 \mathrm{pmol} / \mathrm{mol}$ & Apr 1992 & $\mathrm{ARC}$ & Leaitch et al. (1994) \\
\hline $\mathrm{CHBrCl}_{2}$ & mean: $0.12 \mathrm{pmol} / \mathrm{mol}$ & Sep 1992 & NA & Schall and Heumann (1993) \\
\hline $\mathrm{CHBrCl}_{2}$ & mean: $0.05 \mathrm{pmol} / \mathrm{mol}$ & Feb 1999 & $\mathrm{~nm}$ & Fischer et al. (2002) \\
\hline $\mathrm{CBrClF}_{2}$ & $0.9 \ldots 1.2 \mathrm{pmol} / \mathrm{mol}$ & all 1983 & $\mathrm{BA}$ & Rasmussen and Khalil (1984) \\
\hline \multicolumn{5}{|c|}{ Iodine (inorganic) } \\
\hline gaseous I & $2.2 \mathrm{ng} / \mathrm{m}^{3}(\mathrm{STP})$ & Nov-Dec 1970 & $\mathrm{~mm}$ & Duce et al. (1973) \\
\hline gaseous I & $2.7 \mathrm{ng} / \mathrm{m}^{3}(\mathrm{STP})$ & Nov-Dec 1970 & $\mathrm{sp}$ & Duce et al. (1973) \\
\hline $\mathrm{IO}$ & & $1995-1996$ & NA & Tuckermann et al. (1997) \\
\hline $\mathrm{IO}$ & & 1995-1998 & NA & Wittrock et al. (2000) \\
\hline $\mathrm{IO}$ & up to $10 \mathrm{pmol} / \mathrm{mol}$ & 1999 & $\mathrm{~nm}$ & Frieß et al. (2001) \\
\hline $\mathrm{IO}$ & & May 2000 & $\mathrm{AL}$ & Hönninger (2002) \\
\hline \multicolumn{5}{|c|}{ Iodine (organic) } \\
\hline $\mathrm{CH}_{3} \mathrm{I}$ & & 1982,1983 & $\mathrm{NO}$ & Hov et al. (1984) \\
\hline $\mathrm{CH}_{3} \mathrm{I}$ & $0.9 \ldots 1.4 \mathrm{pmol} / \mathrm{mol}$ & Jan 1992 & $\mathrm{AL}$ & Yokouchi et al. (1994) \\
\hline $\mathrm{CH}_{3} \mathrm{I}$ & $0.2 \ldots 0.6 \mathrm{pmol} / \mathrm{mol}$ & Apr 1992 & $\mathrm{AL}$ & Yokouchi et al. (1994) \\
\hline $\mathrm{CH}_{3} \mathrm{I}$ & mean: $1.04 \mathrm{pmol} / \mathrm{mol}$ & Sep 1992 & NA & Schall and Heumann (1993) \\
\hline $\mathrm{CH}_{3} \mathrm{I}$ & & 2000 & sp & Swanson et al. (2004) \\
\hline $\mathrm{CH}_{2} \mathrm{I}_{2}$ & mean: $0.46 \mathrm{pmol} / \mathrm{mol}$ & Sep 1992 & $\mathrm{NA}$ & Schall and Heumann (1993) \\
\hline $\mathrm{CH}_{2} \mathrm{I}_{2}$ & & Mar 2004 & $\mathrm{HU}$ & Carpenter et al. (2005) \\
\hline $\mathrm{CH}_{2} \mathrm{ICl}$ & mean: $0.07 \mathrm{pmol} / \mathrm{mol}$ & Sep 1992 & $\mathrm{NA}$ & Schall and Heumann (1993) \\
\hline $\mathrm{CH}_{2} \mathrm{ICl}$ & & Mar 2004 & $\mathrm{HU}$ & Carpenter et al. (2005) \\
\hline $\mathrm{CH}_{2} \mathrm{IBr}$ & & Mar 2004 & $\mathrm{HU}$ & Carpenter et al. (2005) \\
\hline $\mathrm{C}_{3} \mathrm{H}_{7} \mathrm{I}$ & mean: $0.20 \mathrm{pmol} / \mathrm{mol}$ & Sep 1992 & NA & Schall and Heumann (1993) \\
\hline $\mathrm{CH}_{3} \mathrm{CHICH}_{3}$ & mean: $2.00 \mathrm{pmol} / \mathrm{mol}$ & Sep 1992 & NA & Schall and Heumann (1993) \\
\hline \multicolumn{5}{|c|}{ Sulfur (inorganic) } \\
\hline $\mathrm{SO}_{2}$ & $5 \ldots 100 \mathrm{pmol} / \mathrm{mol}$ & Jun-Aug 1980 & $\mathrm{NO}$ & Ockelmann and Georgii (1984) \\
\hline $\mathrm{SO}_{2}$ & $5 \ldots 100 \mathrm{pmol} / \mathrm{mol}$ & Jun-Aug 1980 & $\mathrm{ARC}$ & Ockelmann and Georgii (1984) \\
\hline $\mathrm{SO}_{2}$ & $100 \ldots 200 \mathrm{pmol} / \mathrm{mol}$ & Nov/Dec 1981 & IG & Hoff et al. (1983) \\
\hline $\mathrm{SO}_{2}$ & $700 \ldots 1300 \mathrm{pmol} / \mathrm{mol}$ & Feb 1982 & $\mathrm{IG}$ & Hoff et al. (1983) \\
\hline $\mathrm{SO}_{2}$ & $<110 \ldots 590 \mathrm{pmol} / \mathrm{mol}$ & Apr 1982 & IG & Barrie and Hoff (1984) \\
\hline $\mathrm{SO}_{2}$ & $0 \ldots>1000 \mathrm{pmol} / \mathrm{mol}$ & Apr 1983 & $\mathrm{BA}$ & Radke et al. (1984) \\
\hline $\mathrm{SO}_{2}$ & $11 \mathrm{pmol} / \mathrm{mol}$ & Mar-Apr 1986 & ant & Berresheim (1987) \\
\hline $\mathrm{SO}_{2}$ & $6 . \ldots 1600 \mathrm{pmol} / \mathrm{mol}$ & spr 1988 & $\mathrm{AL}$ & Bottenheim et al. (1990) \\
\hline $\mathrm{SO}_{2}$ & & Mar-Apr 1988 & $\mathrm{AL}$ & Bottenheim et al. (1993) \\
\hline
\end{tabular}




\begin{tabular}{|c|c|c|c|c|}
\hline species & value & date & site & reference \\
\hline $\mathrm{SO}_{2}$ & & Jun 1990 & $\mathrm{ARC}$ & Ferek et al. (1995) \\
\hline $\mathrm{SO}_{2}$ & & $1990-2001$ & $\mathrm{SN}$ & Heidam et al. (2004) \\
\hline $\mathrm{SO}_{2}$ & & Jan-Feb 1991 & $\operatorname{tn}$ & Allegrini et al. (1994) \\
\hline $\mathrm{SO}_{2}$ & & May-Oct 1991 & $\mathrm{BA}$ & Ferek et al. (1995) \\
\hline $\mathrm{SO}_{2}$ & $0 \ldots 5000 \mathrm{pmol} / \mathrm{mol}$ & win/spr 1992 & $\mathrm{AL}$ & Barrie et al. (1994a) \\
\hline $\mathrm{SO}_{2}$ & & Apr 1992 & $\mathrm{ARC}$ & Ferek et al. (1995) \\
\hline $\mathrm{SO}_{2}$ & mean: $0.9 \mathrm{nmol} / \mathrm{m}^{3}(\mathrm{STP})$ & Jun-Jul 1993 & $\mathrm{SU}$ & Dibb et al. (1994) \\
\hline $\mathrm{SO}_{2}$ & & Mar-Jun 1994 & $\mathrm{ARC}$ & Jaeschke et al. (1997) \\
\hline $\mathrm{SO}_{2}$ & & Aug 1994 & SU & Dibb et al. (1996) \\
\hline $\mathrm{SO}_{2}$ & & $1995-1996$ & NA & Martinez et al. (1999) \\
\hline $\mathrm{SO}_{2}$ & & 1995,1996 & NA & Tuckermann et al. (1997) \\
\hline $\mathrm{SO}_{2}$ & $0 . .2592 .7 \mathrm{pmol} / \mathrm{mol}$ & 1997-1999 (spr) & NA & Beine et al. (2001) \\
\hline $\mathrm{SO}_{2}$ & & 1998-1999 & $\mathrm{du}$ & $\begin{array}{l}\text { Jourdain and Legrand } \\
\text { (2001),Legrand et al. (2001) }\end{array}$ \\
\hline $\mathrm{SO}_{2}$ & & Mar-Apr 2000 & NA & Hara et al. $(2002 b)$ \\
\hline $\mathrm{SO}_{2}$ & mean: $1487.5 \mathrm{ng} / \mathrm{m}^{3}$ & Feb 2000 & $\mathrm{AL}$ & Ianniello et al. (2002) \\
\hline $\mathrm{SO}_{2}$ & mean: $280.0 \mathrm{ng} / \mathrm{m}^{3}$ & Apr-May 2000 & $\mathrm{AL}$ & Ianniello et al. (2002) \\
\hline $\mathrm{SO}_{2}$ & & 2000 & $\mathrm{sp}$ & Huey et al. (2004) \\
\hline $\mathrm{SO}_{2}$ & & $2002-2005$ & NU & Skov et al. (2006) \\
\hline $\mathrm{H}_{2} \mathrm{SO}_{4}$ & mean: $1.61 \times 10^{6} \mathrm{~cm}^{-3}$ & Feb 1994 & $\mathrm{pa}$ & $\begin{array}{l}\text { Jefferson et al. (1998); Davis et al. } \\
(1998)\end{array}$ \\
\hline $\mathrm{H}_{2} \mathrm{SO}_{4}$ & & 2000 & sp & Mauldin III et al. (2004) \\
\hline $\mathrm{H}_{2} \mathrm{SO}_{4}$ & & Feb-May 2000 & $\mathrm{ARC}$ & Mauldin III et al. (2003) \\
\hline \multicolumn{5}{|c|}{ Sulfur (organic) } \\
\hline $\mathrm{CH}_{3} \mathrm{SCH}_{3}$ & $96 \mathrm{pmol} / \mathrm{mol}$ & Mar-Apr 1986 & ant & Berresheim (1987) \\
\hline $\mathrm{CH}_{3} \mathrm{SCH}_{3}$ & & Jun 1990 & $\mathrm{ARC}$ & Ferek et al. (1995) \\
\hline $\mathrm{CH}_{3} \mathrm{SCH}_{3}$ & & May-Oct 1991 & $\mathrm{BA}$ & Ferek et al. (1995) \\
\hline $\mathrm{CH}_{3} \mathrm{SCH}_{3}$ & $<0.3 \mathrm{pmol} / \mathrm{mol}$ & Jan-Apr 1992 & $\mathrm{AL}$ & Yokouchi et al. (1994) \\
\hline $\mathrm{CH}_{3} \mathrm{SCH}_{3}$ & & Apr 1992 & $\mathrm{ARC}$ & Ferek et al. (1995) \\
\hline $\mathrm{CH}_{3} \mathrm{SCH}_{3}$ & mean: $119 \mathrm{pmol} / \mathrm{mol}$ & Jan-Feb 1994 & $\mathrm{pa}$ & Berresheim et al. (1998) \\
\hline $\mathrm{CH}_{3} \mathrm{SCH}_{3}$ & & 1998-1999 & $\mathrm{du}$ & $\begin{array}{l}\text { Jourdain and Legrand } \\
\text { (2001),Legrand et al. (2001) }\end{array}$ \\
\hline $\mathrm{CH}_{3} \mathrm{SCH}_{3}$ & & 2000 & $\mathrm{sp}$ & Swanson et al. (2004) \\
\hline $\mathrm{CH}_{3} \mathrm{SCH}_{3}$ & mean: $38.1 \mathrm{pmol} / \mathrm{mol}$ & $2004-2005$ & ha & Read et al. (2006) \\
\hline $\mathrm{CH}_{3} \mathrm{SOCH}_{3}$ & & Jan 1994 & $\mathrm{pa}$ & Davis et al. (1998) \\
\hline $\mathrm{CH}_{3} \mathrm{SOCH}_{3}$ & mean: $2.3 \mathrm{pmol} / \mathrm{mol}$ & Jan-Feb 1994 & pa & Berresheim et al. (1998) \\
\hline $\mathrm{CH}_{3} \mathrm{SOCH}_{3}$ & & 1998-1999 & $\mathrm{du}$ & $\begin{array}{l}\text { Jourdain and Legrand } \\
\text { (2001),Legrand et al. (2001) }\end{array}$ \\
\hline $\mathrm{CH}_{3} \mathrm{SO}_{2} \mathrm{CH}_{3}$ & & Jan 1994 & $\mathrm{pa}$ & Davis et al. (1998) \\
\hline $\mathrm{CH}_{3} \mathrm{SO}_{2} \mathrm{CH}_{3}$ & mean: $1.7 \mathrm{pmol} / \mathrm{mol}$ & Jan-Feb 1994 & $\mathrm{pa}$ & Berresheim et al. (1998) \\
\hline OCS & & 1982,1983 & $\mathrm{NO}$ & Hov et al. (1984) \\
\hline MSA (gaseous) & mean: $9.5 \times 10^{5} \mathrm{~cm}^{-3}$ & Feb 1994 & $\mathrm{pa}$ & $\begin{array}{l}\text { Jefferson et al. (1998); Davis et al. } \\
\text { (1998) }\end{array}$ \\
\hline MSA (gaseous) & & Feb-May 2000 & $\mathrm{ARC}$ & Mauldin III et al. (2003) \\
\hline \multicolumn{5}{|c|}{ Mercury } \\
\hline $\mathrm{Hg}$ & $\begin{array}{l}\text { annual mean: } 1.50 \ldots 1.79 \\
\mathrm{ng} / \mathrm{m}^{3}\end{array}$ & 1994-2002 & NA & Berg et al. (2004) \\
\hline $\mathrm{Hg}(\mathrm{TGM})$ & & 1995 & $\mathrm{AL}$ & Schroeder et al. (1998) \\
\hline $\mathrm{Hg}(\mathrm{GEM})$ & & 1995-1999 & $\mathrm{AL}$ & Banic et al. (2003) \\
\hline $\mathrm{Hg}(\mathrm{GEM})$ & median: $1.58 \mathrm{ng} / \mathrm{m}^{3}$ & $1995-2002$ & $\mathrm{AL}$ & Steffen et al. (2005) \\
\hline $\mathrm{Hg}$ & mean: $1.26 \mathrm{ng} / \mathrm{m}^{3}$ & 1996-1997 & $\mathrm{PS}$ & Berg et al. (2001) \\
\hline $\mathrm{Hg}$ & mean: $1.43 \mathrm{ng} / \mathrm{m}^{3}$ & 1996-1997 & NA & Berg et al. (2001) \\
\hline $\mathrm{Hg}(\mathrm{GEM})$ & & Apr-May 1998 & $\mathrm{AL}$ & Lu et al. (2001) \\
\hline $\mathrm{Hg}(\mathrm{GEM})$ & & Apr 1998 & BS & Banic et al. (2003) \\
\hline $\mathrm{Hg}(\mathrm{GEM})$ & & $1998-2002$ & $\mathrm{SN}$ & Skov et al. (2004) \\
\hline $\mathrm{Hg}(\mathrm{GEM})$ & median: $1.8 \mathrm{ng} / \mathrm{m}^{3}$ & $1999-2000$ & $\mathrm{KU}$ & Steffen et al. (2005) \\
\hline $\mathrm{Hg}$ (RGM) & & $1999-2000$ & $\mathrm{BA}$ & Lindberg et al. (2001) \\
\hline $\mathrm{Hg}(\mathrm{GEM})$ & & $1999-2000$ & $\mathrm{BA}$ & Lindberg et al. (2001) \\
\hline
\end{tabular}




\begin{tabular}{|c|c|c|c|c|}
\hline species & value & date & site & reference \\
\hline Hg (GEM) & & 1999-2001 & $\mathrm{BA}$ & Lindberg et al. (2002) \\
\hline $\mathrm{Hg}$ (RGM) & & $1999-2001$ & $\mathrm{BA}$ & Lindberg et al. (2002) \\
\hline $\mathrm{Hg}$ (GEM) & & $1999-2001$ & $\mathrm{SN}$ & Heidam et al. (2004) \\
\hline $\mathrm{Hg}$ (GEM) & median: $1.47 \mathrm{ng} / \mathrm{m}^{3}$ & 2000 & NA & Berg et al. (2003) \\
\hline Hg (RGM) & & 2000 & NA & Berg et al. (2003) \\
\hline $\mathrm{Hg}$ (GEM) & & Feb-May 2000 & $\mathrm{AL}$ & Steffen et al. (2002) \\
\hline $\mathrm{Hg}$ & & Feb-Mar 2000 & $\mathrm{AL}$ & Bottenheim et al. (2002b) \\
\hline $\mathrm{Hg}(\mathrm{TGM})$ & & $2000-2001$ & $\mathrm{~nm}$ & Ebinghaus et al. (2002) \\
\hline $\mathrm{Hg}$ (GEM) & median: $1.7 \mathrm{ng} / \mathrm{m}^{3}$ & 2001-2003 & $\mathrm{AM}$ & Steffen et al. (2005) \\
\hline $\mathrm{Hg}$ (GEM) & & Mar 2002 & $\mathrm{SN}$ & Ferrari et al. (2004) \\
\hline $\mathrm{Hg}$ (GEM,RGM) & & Apr 2002 & $\mathrm{AL}$ & Steffen et al. (2003) \\
\hline $\mathrm{Hg}$ (TGM,RGM) & & Apr-May 2002 & NA & Sommar et al. (2007) \\
\hline $\mathrm{Hg}$ (GEM,RGM) & & Apr-May 2003 & NA & Sprovieri et al. $(2005 \mathrm{a}, \mathrm{b})$ \\
\hline $\mathrm{Hg}$ (GEM) & & Apr-May 2003 & NA & $\begin{array}{l}\text { Aspmo et al. (2005); Gauchard } \\
\text { et al. (2005) }\end{array}$ \\
\hline $\mathrm{Hg}(\mathrm{RGM})$ & & Apr-May 2003 & NA & $\begin{array}{l}\text { Aspmo et al. (2005); Gauchard } \\
\text { et al. }(2005)\end{array}$ \\
\hline $\mathrm{Hg}(\mathrm{TGM})$ & & Mar 2004 & $\mathrm{HU}$ & Carpenter et al. (2005) \\
\hline $\mathrm{Hg}$ (GEM) & & Mar-Apr 2005 & $\mathrm{BA}$ & Tackett et al. (2006) \\
\hline
\end{tabular}




\begin{tabular}{|c|c|c|c|c|}
\hline species & value & date & site & reference \\
\hline \multicolumn{5}{|c|}{$* * * * * \quad \mathrm{~A} \mathrm{e}$} \\
\hline \multicolumn{5}{|c|}{ Nitrogen } \\
\hline $\mathrm{NH}_{4}^{+}$ & & 1979-1984 & $\mathrm{ARC}$ & Barrie and Hoff (1985) \\
\hline $\mathrm{NH}_{4}^{+}$ & & 1980-1995 & $\mathrm{AL}$ & Sirois and Barrie (1999) \\
\hline $\mathrm{NH}_{4}^{+}$ & & Apr-May 1980 & $\mathrm{MB}, \mathrm{IG}$ & Barrie et al. (1981) \\
\hline $\mathrm{NH}_{4}^{+}$ & $115 \ldots 1030 \mathrm{ng} / \mathrm{m}^{3}$ & Feb 1982 & $\mathrm{IG}$ & Hoff et al. (1983) \\
\hline $\mathrm{NH}_{4}^{+}$ & & Mar-May 1986 & $\mathrm{BA}$ & Li and Winchester (1989a,b) \\
\hline $\mathrm{NH}_{4}^{+}$ & $20 \ldots 701 \mathrm{ng} / \mathrm{m}^{3}$ & spr 1988 & $\mathrm{AL}$ & Bottenheim et al. (1990) \\
\hline $\mathrm{NH}_{4}^{+}$ & mean: $180 \mathrm{ng} / \mathrm{m}^{3}$ & May 1989 & $\mathrm{AL}$ & Kieser et al. (1993) \\
\hline $\mathrm{NH}_{4}^{+}$ & & $1990-2001$ & SN & Heidam et al. (2004) \\
\hline $\mathrm{NH}_{4}^{+}$ & & $1991-1995$ & $\mathrm{du}$ & Legrand et al. (1998) \\
\hline $\mathrm{NH}_{4}^{+}$ & & $1991-1995$ & $\mathrm{~nm}$ & Legrand et al. (1998) \\
\hline $\mathrm{NH}_{4}^{+}$ & & 1991-1999 & $\mathrm{du}$ & Jourdain and Legrand (2002) \\
\hline $\mathrm{NH}_{4}^{+}$ & mean: $69 \mathrm{ng} / \mathrm{m}^{3}$ & May-Jul 1993 & SU & Bergin et al. (1995) \\
\hline $\mathrm{NH}_{4}^{+}$ & & Jan 1994 & $\mathrm{du}$ & Legrand et al. (1998) \\
\hline $\mathrm{NH}_{4}^{+}$ & & Aug 1994 & $\mathrm{SU}$ & Dibb et al. (1996) \\
\hline $\mathrm{NH}_{4}^{+}$ & $0 . \ldots 969.6 \mathrm{pmol} / \mathrm{mol}$ & 1997-1999 (spr) & NA & Beine et al. (2001) \\
\hline $\mathrm{NH}_{4}^{+}$ & mean: $124.5 \mathrm{ng} / \mathrm{m}^{3}$ & Feb 2000 & $\mathrm{AL}$ & Ianniello et al. (2002) \\
\hline $\mathrm{NH}_{4}^{+}$ & mean: $89.9 \mathrm{ng} / \mathrm{m}^{3}$ & Apr-May 2000 & $\mathrm{AL}$ & Ianniello et al. (2002) \\
\hline $\mathrm{NH}_{4}^{+}$ & mean: $105 \mathrm{ng} / \mathrm{m}^{3}$ & Apr-May 2001 & NA & Teinilä et al. (2003) \\
\hline $\mathrm{NH}_{4}^{+}$ & mean: $108 \mathrm{ng} / \mathrm{m}^{3}$ & Feb-Mar 2001 & NA & Teinilä et al. (2003) \\
\hline $\mathrm{NO}_{2}^{-}$ & $<23 \mathrm{ng} / \mathrm{m}^{3}$ & win/spr 1992 & $\mathrm{AL}$ & Li (1994) \\
\hline $\mathrm{NO}_{2}^{-}$ & $<$DL. . $20 \mathrm{pmol} / \mathrm{mol}$ & Feb-May 2001 & NA & Beine et al. (2003) \\
\hline $\mathrm{NO}_{2}^{-}$ & $0 \ldots 260.1 \mathrm{pmol} / \mathrm{mol}$ & 1997-1999 (spr) & NA & Beine et al. (2001) \\
\hline $\mathrm{NO}_{3}^{-}$ & & $1979-1984$ & $\mathrm{ARC}$ & Barrie and Hoff (1985) \\
\hline $\mathrm{NO}_{3}^{-}$ & & Apr-May 1980 & $\mathrm{MB}, \mathrm{IG}$ & Barrie et al. (1981) \\
\hline $\mathrm{NO}_{3}^{-}$ & $10 \ldots 100 \mathrm{ng} / \mathrm{m}^{3}$ & $1980-1988$ & $\mathrm{AL}$ & Barrie and Delmas (1994) \\
\hline $\mathrm{NO}_{3}^{-}$ & & 1980-1995 & $\mathrm{AL}$ & Sirois and Barrie (1999) \\
\hline $\mathrm{NO}_{3}^{-}$ & $17 \ldots 260 \mathrm{ng} / \mathrm{m}^{3}$ & Feb 1982 & IG & Hoff et al. (1983) \\
\hline $\mathrm{NO}_{3}^{-}$ & $30 \mathrm{ng} / \mathrm{m}^{3}$ & Apr 1983 & $\mathrm{BA}$ & Radke et al. (1984) \\
\hline $\mathrm{NO}_{3}^{-}$ & & $1983-1996$ & $\mathrm{~nm}$ & Wagenbach et al. (1998b) \\
\hline $\mathrm{NO}_{3}^{-}$ & & Mar-May 1986 & $\mathrm{BA}$ & Li and Winchester $(1989 a, b)$ \\
\hline $\mathrm{NO}_{3}^{-}$ & $7.1 \ldots 245.7 \mathrm{ng} / \mathrm{m}^{3}$ & spr 1988 & $\mathrm{AL}$ & Bottenheim et al. (1990) \\
\hline $\mathrm{NO}_{3}^{-}$ & & Mar-Apr 1988 & $\mathrm{AL}$ & Bottenheim et al. (1993) \\
\hline $\mathrm{NO}_{3}^{-}$ & mean: $189 \mathrm{ng} / \mathrm{m}^{3}$ & May 1989 & $\mathrm{AL}$ & Kieser et al. (1993) \\
\hline $\mathrm{NO}_{3}^{-}$ & & Jan-Feb 1991 & tn & Allegrini et al. (1994) \\
\hline $\mathrm{NO}_{3}^{-}$ & & 1991-1995 & $\mathrm{du}$ & Wagenbach et al. (1998b) \\
\hline $\mathrm{NO}_{3}^{-}$ & & $1991-2001$ & $\mathrm{du}$ & Jourdain and Legrand (2002) \\
\hline $\mathrm{NO}_{3}^{-}$ & $30 \ldots 400 \mathrm{ng} / \mathrm{m}^{3}$ & win/spr 1992 & $\mathrm{AL}$ & Barrie et al. (1994a) \\
\hline $\mathrm{NO}_{3}^{-}$ & mean: $26 \mathrm{ng} / \mathrm{m}^{3}$ & May-Jul 1993 & $\mathrm{SU}$ & Bergin et al. (1995) \\
\hline $\mathrm{NO}_{3}^{-}$ & mean: $0.06 \mathrm{nmol} / \mathrm{m}^{3}(\mathrm{STP})$ & Jun-Jul 1993 & SU & Dibb et al. (1994) \\
\hline $\mathrm{NO}_{3}^{-}$ & & Jan 1994 & $\mathrm{du}$ & Legrand et al. (1998) \\
\hline $\mathrm{NO}_{3}^{-}$ & & Mar-Jun 1994 & $\mathrm{ARC}$ & Jaeschke et al. (1997) \\
\hline $\mathrm{NO}_{3}^{-}$ & & Aug 1994 & $\mathrm{SU}$ & Dibb et al. (1996) \\
\hline $\mathrm{NO}_{3}^{-}$ & & 1996-1999 & NA & Hara et al. (2002a) \\
\hline $\mathrm{NO}_{3}^{-}$ & mean: $4 \mathrm{pmol} / \mathrm{mol}$ & Jan-Mar 1997 & $\mathrm{~nm}$ & Jones et al. (1999) \\
\hline $\mathrm{NO}_{3}^{-}$ & $0 \ldots 3537.1 \mathrm{pmol} / \mathrm{mol}$ & $1997-1999$ (spr) & NA & Beine et al. (2001) \\
\hline $\mathrm{NO}_{3}^{-}$ & mean: $39 \mathrm{ng} / \mathrm{m}^{3}$ & $1998-1999$ & $\mathrm{sp}$ & Arimoto et al. (2001) \\
\hline $\mathrm{NO}_{3}^{-}$ & & 1998,2000 & $\mathrm{sp}$ & Arimoto et al. (2004a) \\
\hline $\mathrm{NO}_{3}^{-}$ & mean: $4.2 \mathrm{pmol} / \mathrm{mol}$ & Jan-Feb 1999 & $\mathrm{~nm}$ & Jacobi et al. (2000) \\
\hline $\mathrm{NO}_{3}^{-}$ & mean: $81.3 \mathrm{ng} / \mathrm{m}^{3}$ & Feb 2000 & $\mathrm{AL}$ & Ianniello et al. (2002) \\
\hline $\mathrm{NO}_{3}^{-}$ & & Mar-Apr 2000 & NA & Hara et al. (2002b) \\
\hline $\mathrm{NO}_{3}^{-}$ & mean: $137.4 \mathrm{ng} / \mathrm{m}^{3}$ & Apr-May 2000 & $\mathrm{AL}$ & Ianniello et al. (2002) \\
\hline $\mathrm{NO}_{3}^{-}$ & & 2001 & $\mathrm{du}$ & Savarino et al. (2006) \\
\hline $\mathrm{NO}_{3}^{-}$ & & Jan-Dec 2001 & ha & Rankin and Wolff (2003) \\
\hline $\mathrm{NO}_{3}^{-}$ & mean: $59 \mathrm{ng} / \mathrm{m}^{3}$ & Feb-Mar 2001 & NA & Teinilä et al. (2003) \\
\hline $\mathrm{NO}_{3}^{-}$ & $7 \ldots 80 \mathrm{pmol} / \mathrm{mol}$ & Feb-May 2001 & NA & Beine et al. (2003) \\
\hline $\mathrm{NO}_{3}^{-}$ & mean: $65 \mathrm{ng} / \mathrm{m}^{3}$ & Apr-May 2001 & NA & Teinilä et al. (2003) \\
\hline
\end{tabular}




\begin{tabular}{|c|c|c|c|c|}
\hline species & value & date & site & reference \\
\hline $\mathrm{NO}_{3}^{-}$ & & spr 2004 & $\mathrm{AL}$ & Morin et al. (2006) \\
\hline \multicolumn{5}{|c|}{ Fluorine } \\
\hline $\mathrm{F}$ & & $1979-1984$ & $\mathrm{ARC}$ & Barrie and Hoff (1985) \\
\hline \multicolumn{5}{|c|}{ Chlorine } \\
\hline $\mathrm{Cl}^{-}$ & & Apr-May 1980 & $\mathrm{MB}, \mathrm{IG}$ & Barrie et al. (1981) \\
\hline $\mathrm{Cl}^{-}$ & & 1980-1995 & $\mathrm{AL}$ & Sirois and Barrie (1999) \\
\hline $\mathrm{Cl}^{-}$ & $149 \ldots 563 \mathrm{ng} / \mathrm{m}^{3}$ & Feb 1982 & IG & Hoff et al. (1983) \\
\hline $\mathrm{Cl}^{-}$ & $230 \mathrm{ng} / \mathrm{m}^{3}$ & Apr 1983 & $\mathrm{BA}$ & Radke et al. (1984) \\
\hline $\mathrm{Cl}^{-}$ & & $1983-1996$ & $\mathrm{~nm}$ & Wagenbach et al. (1998a) \\
\hline $\mathrm{Cl}^{-}$ & & Mar-May 1986 & $\mathrm{BA}$ & Li and Winchester $(1989 a, b)$ \\
\hline $\mathrm{Cl}^{-}$ & mean: $286 \mathrm{ng} / \mathrm{m}^{3}$ & May 1989 & $\mathrm{AL}$ & Kieser et al. (1993) \\
\hline $\mathrm{Cl}^{-}$ & & $1991-1993$ & ha & Wagenbach et al. (1998a) \\
\hline $\mathrm{Cl}^{-}$ & & 1991-1995 & $\mathrm{du}$ & Wagenbach et al. (1998a) \\
\hline $\mathrm{Cl}^{-}$ & & $1991-2001$ & $\mathrm{du}$ & Jourdain and Legrand (2002) \\
\hline $\mathrm{Cl}^{-}$ & & Jan 1994 & $\mathrm{du}$ & Legrand et al. (1998) \\
\hline $\mathrm{Cl}^{-}$ & & Aug 1994 & $\mathrm{SU}$ & Dibb et al. (1996) \\
\hline $\mathrm{Cl}^{-}$ & & 1996-1999 & NA & Hara et al. (2002a) \\
\hline $\mathrm{Cl}^{-}$ & $0 \ldots 1391.0 \mathrm{pmol} / \mathrm{mol}$ & $1997-1999$ (spr) & NA & Beine et al. (2001) \\
\hline $\mathrm{Cl}^{-}$ & & 1998,2000 & $\mathrm{sp}$ & Arimoto et al. (2004a) \\
\hline $\mathrm{Cl}^{-}$ & mean: $73.9 \mathrm{ng} / \mathrm{m}^{3}$ & Feb 2000 & $\mathrm{AL}$ & Ianniello et al. (2002) \\
\hline $\mathrm{Cl}^{-}$ & mean: $220.9 \mathrm{ng} / \mathrm{m}^{3}$ & Apr-May 2000 & $\mathrm{AL}$ & Ianniello et al. (2002) \\
\hline $\mathrm{Cl}^{-}$ & & Jan-Dec 2001 & ha & Rankin and Wolff (2003) \\
\hline $\mathrm{Cl}^{-}$ & mean: $449 \mathrm{ng} / \mathrm{m}^{3}$ & Feb-Mar 2001 & NA & Teinilä et al. (2003) \\
\hline $\mathrm{Cl}^{-}$ & mean: $373 \mathrm{ng} / \mathrm{m}^{3}$ & Apr-May 2001 & NA & Teinilä et al. (2003) \\
\hline $\mathrm{Cl}$ & $<20 \ldots 4000 \mathrm{ng} / \mathrm{m}^{3}(\mathrm{STP})$ & Jan 1965 & $\mathrm{BA}$ & Duce et al. (1966) \\
\hline $\mathrm{Cl}$ & $<0.01 \mathrm{ng} / \mathrm{m}^{3}(\mathrm{STP})$ & Nov-Dec 1970 & $\mathrm{sp}$ & Duce et al. (1973) \\
\hline $\mathrm{Cl}$ & $0.07 \mathrm{ng} / \mathrm{m}^{3}(\mathrm{STP})$ & Nov-Dec 1970 & $\mathrm{~mm}$ & Duce et al. (1973) \\
\hline $\mathrm{Cl}$ & & $1976-1980$ & $\mathrm{BA}$ & Berg et al. (1983) \\
\hline $\mathrm{Cl}$ & & 1979-1980 & $\mathrm{ARC}$ & Heidam (1985) \\
\hline $\mathrm{Cl}$ & & 1979-1984 & $\mathrm{ARC}$ & Barrie and Hoff (1985) \\
\hline $\mathrm{Cl}$ & $87.9 \ldots 411 \mathrm{ng} / \mathrm{m}^{3}$ & Feb 1982 & $\mathrm{IG}$ & Hoff et al. (1983) \\
\hline $\mathrm{Cl}$ & & Aug-Sep 1983 & $\mathrm{ARC}$ & Pacyna and Ottar (1985) \\
\hline $\mathrm{Cl}$ & & spr 1983 & $\mathrm{ARC}$ & Winchester et al. (1985) \\
\hline $\mathrm{Cl}$ & & $1983-1986$ & $\mathrm{NA}, \mathrm{NO}$ & Maenhaut et al. (1989) \\
\hline $\mathrm{Cl}$ & $<20 \ldots 302 \mathrm{ng} / \mathrm{m}^{3}$ & spr 1983 & $\mathrm{ARC}$ & Cahill and Eldred (1984) \\
\hline $\mathrm{Cl}$ & & $1984-1987$ & $\mathrm{PF}$ & Sturges and Shaw (1993) \\
\hline $\mathrm{Cl}$ & $21 \ldots 2575 \mathrm{ng} / \mathrm{m}^{3}$ & spr 1988 & $\mathrm{AL}$ & Bottenheim et al. (1990) \\
\hline $\mathrm{Cl}$ & $0.028 \ldots 55 \mathrm{ng} / \mathrm{m}^{3}$ & $1988-1989$ & DY & Mosher et al. (1993) \\
\hline $\mathrm{Cl}$ & $0.53 \ldots 20 \mathrm{ng} / \mathrm{m}^{3}$ & 1988-1989 & $\mathrm{SU}$ & Mosher et al. (1993) \\
\hline $\mathrm{Cl}$ & $0 \ldots 1200 \mathrm{ng} / \mathrm{m}^{3}$ & win/spr 1992 & $\mathrm{AL}$ & Barrie et al. (1994b) \\
\hline $\mathrm{Cl}$ & mean: $34 \mathrm{ng} / \mathrm{m}^{3}$ & $1998-1999$ & $\mathrm{sp}$ & Arimoto et al. (2001) \\
\hline $\mathrm{Cl}$ & mean: $519.43 \mathrm{ng} / \mathrm{m}^{3}$ & $2002-2005$ & NU & Skov et al. (2006) \\
\hline \multicolumn{5}{|c|}{ Bromine } \\
\hline $\mathrm{Br}^{-}$ & & 1983-1996 & $\mathrm{nm}$ & Wagenbach et al. (1998a) \\
\hline $\mathrm{Br}^{-}$ & & Mar-May 1986 & $\mathrm{BA}$ & $\mathrm{Li}$ and Winchester $(1989 \mathrm{a}, \mathrm{b})$ \\
\hline $\mathrm{Br}^{-}$ & $1 \ldots 54 \mathrm{ng} / \mathrm{m}^{3}$ & spr 1988 & $\mathrm{AL}$ & Bottenheim et al. (1990) \\
\hline $\mathrm{Br}^{-}$ & mean: $45 \mathrm{ng} / \mathrm{m}^{3}$ & May 1989 & $\mathrm{AL}$ & Kieser et al. (1993) \\
\hline $\mathrm{Br}^{-}$ & & 1991-1995 & $\mathrm{du}$ & Wagenbach et al. (1998a) \\
\hline $\mathrm{Br}^{-}$ & $10 \ldots 20 \mathrm{ng} / \mathrm{m}^{3}$ & win 1992 & $\mathrm{AL}$ & Li et al. (1994) \\
\hline $\mathrm{Br}^{-}$ & $20 \ldots 120 \mathrm{ng} / \mathrm{m}^{3}$ & spr 1992 & $\mathrm{AL}$ & Li et al. (1994) \\
\hline $\mathrm{Br}^{-}$ & & 1996-1999 & NA & Hara et al. (2002a) \\
\hline $\mathrm{Br}^{-}$ & mean: $13.1 \mathrm{ng} / \mathrm{m}^{3}$ & Apr-May 2000 & $\mathrm{AL}$ & Ianniello et al. (2002) \\
\hline $\mathrm{Br}^{-}$ & mean: $5.2 \mathrm{ng} / \mathrm{m}^{3}$ & Feb 2000 & $\mathrm{AL}$ & Ianniello et al. (2002) \\
\hline $\mathrm{Br}$ & $1 \ldots 30 \mathrm{ng} / \mathrm{m}^{3}(\mathrm{STP})$ & Jan 1965 & $\mathrm{BA}$ & Duce et al. (1966) \\
\hline $\mathrm{Br}$ & $0.63 \mathrm{ng} / \mathrm{m}^{3}(\mathrm{STP})$ & Oct 1970 & $\mathrm{sp}$ & Zoller et al. (1974) \\
\hline $\mathrm{Br}$ & $0.43 \mathrm{ng} / \mathrm{m}^{3}(\mathrm{STP})$ & Nov-Dec 1970 & $\mathrm{sp}$ & Duce et al. (1973) \\
\hline $\mathrm{Br}$ & $0.96 \mathrm{ng} / \mathrm{m}^{3}(\mathrm{STP})$ & Nov-Dec 1970 & $\mathrm{~mm}$ & Duce et al. (1973) \\
\hline
\end{tabular}




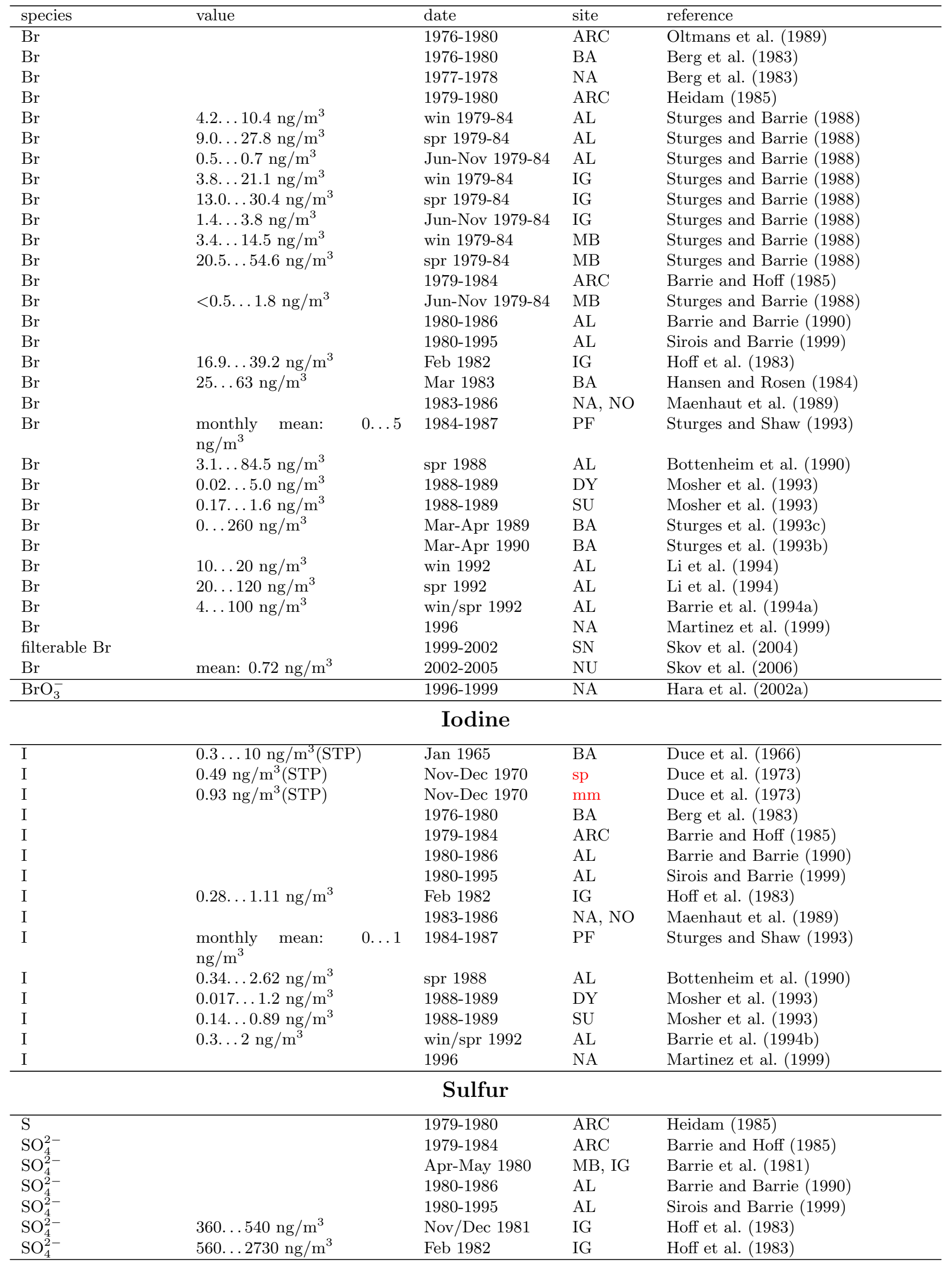




\begin{tabular}{|c|c|c|c|c|}
\hline species & value & date & site & reference \\
\hline $\mathrm{SO}_{4}^{2-}$ & $1460 \ldots 3810 \mathrm{ng} / \mathrm{m}^{3}$ & Apr 1982 & IG & Barrie and Hoff (1984) \\
\hline $\mathrm{SO}_{4}^{2-}$ & $384 \ldots 4380 \mathrm{ng} / \mathrm{m}^{3}$ & Feb 1982 & IG & Hoff et al. (1983) \\
\hline $\mathrm{S}^{4}$ & & Aug-Sep 1983 & $\mathrm{ARC}$ & Pacyna and Ottar (1985) \\
\hline S & $185 \ldots 1070 \mathrm{ng} / \mathrm{m}^{3}$ & spr 1983 & $\mathrm{ARC}$ & Cahill and Eldred (1984) \\
\hline $\mathrm{S}$ & & spr 1983 & $\mathrm{ARC}$ & Winchester et al. (1985) \\
\hline $\mathrm{SO}_{4}^{2-}$ & mean: $3200 \mathrm{ng} / \mathrm{m}^{3}$ & Mar-Apr 1983 & $\mathrm{BA}$ & Lazrus and Ferek (1984) \\
\hline $\mathrm{SO}_{4}^{2-}$ & $2600 \mathrm{ng} / \mathrm{m}^{3}$ & Apr 1983 & $\mathrm{BA}$ & Radke et al. (1984) \\
\hline $\mathrm{S}$ & & $1983-1986$ & $\mathrm{NA}, \mathrm{NO}$ & Maenhaut et al. (1989) \\
\hline $\mathrm{nss}-\mathrm{SO}_{4}^{2-}$ & & 1983-1994 & $\mathrm{nm}$ & Minikin et al. (1998) \\
\hline $\mathrm{nss}-\mathrm{SO}_{4}^{2-}$ & & 1983-1995 & $\mathrm{nm}$ & Legrand and Pasteur (1998) \\
\hline $\mathrm{SO}_{4}^{2-}$ & & 1983-1996 & $\mathrm{nm}$ & Wagenbach et al. (1998a) \\
\hline $\mathrm{SO}_{4}^{2-}$ & & Mar-May 1986 & $\mathrm{BA}$ & Li and Winchester $(1989 \mathrm{a}, \mathrm{b})$ \\
\hline $\mathrm{nss}-\mathrm{SO}_{4}^{2-}$ & $0.31 \mathrm{nmol} / \mathrm{m}^{3}$ & Mar-Apr 1986 & ant & Berresheim (1987) \\
\hline $\mathrm{SO}_{4}^{2-}$ & $440 \ldots 5870 \mathrm{ng} / \mathrm{m}^{3}$ & spr 1988 & $\mathrm{AL}$ & Bottenheim et al. (1990) \\
\hline $\mathrm{SO}_{4}^{2-}$ & & Mar-Apr 1988 & $\mathrm{AL}$ & Bottenheim et al. (1993) \\
\hline $\mathrm{SO}_{4}^{2-}$ & mean: $82 \mathrm{ng} / \mathrm{m}^{3}$ & $1988-1989$ & DY & $\begin{array}{l}\text { Davidson et al. (1993b,a); Jaffrezo } \\
\text { et al. (1994) }\end{array}$ \\
\hline $\mathrm{SO}_{4}^{2-}$ & mean: $1450 \mathrm{ng} / \mathrm{m}^{3}$ & May 1989 & $\mathrm{AL}$ & Kieser et al. (1993) \\
\hline $\mathrm{nss}-\mathrm{SO}_{4}^{2-}$ & & Jun 1990 & $\mathrm{ARC}$ & Ferek et al. (1995) \\
\hline $\mathrm{SO}_{4}^{2-}$ & & 1990-1991 & $\mathrm{SU}$ & Jaffrezo et al. (1994) \\
\hline $\mathrm{SO}_{4}^{2-}$ & & $1990-2001$ & $\mathrm{SN}$ & Heidam et al. (2004) \\
\hline $\mathrm{SO}_{4}^{2-}$ & & Jan-Feb 1991 & $\operatorname{tn}$ & Allegrini et al. (1994) \\
\hline $\mathrm{nss}-\mathrm{SO}_{4}^{2-}$ & & 1991-1992 & ha & Minikin et al. (1998) \\
\hline nss $-\mathrm{SO}_{4}^{2-}$ & & 1991-1992 & ha & Legrand and Pasteur (1998) \\
\hline $\mathrm{SO}_{4}^{2-}$ & & 1991-1993 & ha & Wagenbach et al. (1998a) \\
\hline $\mathrm{nss}-\mathrm{SO}_{4}^{2-}$ & & 1991-1995 & $\mathrm{du}$ & Minikin et al. (1998) \\
\hline $\mathrm{SO}_{4}^{2-}$ & & 1991-1995 & $\mathrm{du}$ & Wagenbach et al. (1998a) \\
\hline $\mathrm{nss}-\mathrm{SO}_{4}^{2-}$ & & 1991-1995 & $\mathrm{nm}$ & Legrand et al. (1998) \\
\hline $\mathrm{nss}-\mathrm{SO}_{4}^{\frac{4}{2}-}$ & & 1991-1996 & $\mathrm{du}$ & Legrand and Pasteur (1998) \\
\hline $\mathrm{SO}_{4}^{2-}$ & & 1991-2001 & $\mathrm{du}$ & Jourdain and Legrand (2002) \\
\hline $\mathrm{SO}_{4}^{2-}$ & $720 \ldots 7700 \mathrm{ng} / \mathrm{m}^{3}$ & win/spr 1992 & $\mathrm{AL}$ & Barrie et al. (1994a) \\
\hline $\mathrm{nss}-\mathrm{SO}_{4}^{2-}$ & & Apr 1992 & $\mathrm{ARC}$ & Ferek et al. (1995) \\
\hline $\mathrm{SO}_{4}^{2-}$ & mean: $120 \mathrm{ng} / \mathrm{m}^{3}$ & Jun-Jul 1992 & $\mathrm{SU}$ & Bergin et al. (1994) \\
\hline $\mathrm{SO}_{4}^{2-}$ & & Mar-Jun 1993 & NA & Solberg et al. (1996b) \\
\hline $\mathrm{SO}_{4}^{2-}$ & mean: $402 \mathrm{ng} / \mathrm{m}^{3}$ & May-Jul 1993 & SU & Bergin et al. (1995) \\
\hline $\mathrm{SO}_{4}^{2-}$ & mean: $3.0 \mathrm{nmol} / \mathrm{m}^{3}(\mathrm{STP})$ & Jun-Jul 1993 & SU & Dibb et al. (1994) \\
\hline $\mathrm{SO}_{4}^{2-}$ & & Jan 1994 & $\mathrm{du}$ & Legrand et al. (1998) \\
\hline $\mathrm{nss}-\mathrm{SO}_{4}^{2-}$ & mean: $69.1 \mathrm{pmol} / \mathrm{mol}$ & Jan-Feb 1994 & $\mathrm{pa}$ & Berresheim et al. (1998) \\
\hline $\mathrm{SO}_{4}^{2-}$ & & Mar-Jun 1994 & $\mathrm{ARC}$ & Jaeschke et al. (1997) \\
\hline $\mathrm{SO}_{4}^{2-}$ & & Aug 1994 & $\mathrm{SU}$ & Dibb et al. (1996) \\
\hline $\mathrm{SO}_{4}^{2-}$ & $0 \ldots 611.1 \mathrm{pmol} / \mathrm{mol}$ & 1997-1999 (spr) & NA & Beine et al. (2001) \\
\hline $\mathrm{nss}-\mathrm{SO}_{4}^{2-}$ & & 1998-1999 & du & $\begin{array}{l}\text { Jourdain and Legrand } \\
\text { (2001),Legrand et al. (2001) }\end{array}$ \\
\hline $\mathrm{SO}_{4}^{2-}$ & mean: $224 \mathrm{ng} / \mathrm{m}^{3}$ & 1998-1999 & sp & Arimoto et al. (2001) \\
\hline $\mathrm{SO}_{4}^{2-}$ & & 1998,2000 & $\mathrm{sp}$ & Arimoto et al. (2004a) \\
\hline $\mathrm{SO}_{4}^{2-}$ & mean: $1111.1 \mathrm{ng} / \mathrm{m}^{3}$ & Feb 2000 & $\mathrm{AL}$ & Ianniello et al. (2002) \\
\hline $\mathrm{SO}_{4}^{2-}$ & mean: $746.3 \mathrm{ng} / \mathrm{m}^{3}$ & Apr-May 2000 & $\mathrm{AL}$ & Ianniello et al. (2002) \\
\hline $\mathrm{SO}_{4}^{2-}$ & & Mar-Apr 2000 & NA & Hara et al. (2002b) \\
\hline $\mathrm{nss}-\mathrm{SO}_{4}^{2-}$ & & Jan-Dec 2001 & ha & Rankin and Wolff (2003) \\
\hline nss $-\mathrm{SO}_{4}^{2-}$ & mean: $929 \mathrm{ng} / \mathrm{m}^{3}$ & Feb-Mar 2001 & NA & Teinilä et al. (2003) \\
\hline $\mathrm{nss}-\mathrm{SO}_{4}^{2-}$ & mean: $1430 \mathrm{ng} / \mathrm{m}^{3}$ & Apr-May 2001 & NA & Teinilä et al. (2003) \\
\hline S & mean: $119.31 \mathrm{ng} / \mathrm{m}^{3}$ & $2002-2005$ & $\mathrm{NU}$ & Skov et al. (2006) \\
\hline MSA & & $1980-1995$ & $\mathrm{AL}$ & Sirois and Barrie (1999) \\
\hline MSA & & $1983-1994$ & $\mathrm{~nm}$ & Minikin et al. (1998) \\
\hline MSA & & 1983-1995 & $\mathrm{nm}$ & Legrand and Pasteur (1998) \\
\hline MSA & $0.3 \ldots 6 \mathrm{pmol} / \mathrm{mol}$ & 1986-1988 & $\mathrm{AL}$ & Li et al. (1993) \\
\hline MSA & & Mar-May 1986 & $\mathrm{BA}$ & Li and Winchester $(1989 \mathrm{a}, \mathrm{b})$ \\
\hline MSA & $0.27 \mathrm{nmol} / \mathrm{m}^{3}$ & Mar-Apr 1986 & ant & Berresheim (1987) \\
\hline MSA & & sum 1988 & $\mathrm{ARC}$ & Li et al. (1993) \\
\hline
\end{tabular}




\begin{tabular}{|c|c|c|c|c|}
\hline species & value & date & site & reference \\
\hline MSA & mean: $0.58 \mathrm{ng} / \mathrm{m}^{3}$ & 1988-1989 & DY & $\begin{array}{l}\text { Davidson et al. (1993b,a); Jaffrezo } \\
\text { et al. (1994); Li et al. (1993) }\end{array}$ \\
\hline MSA & $1 \ldots 25 \mathrm{pmol} / \mathrm{mol}$ & Mar-Apr 1989 & $\mathrm{BA}$ & Li et al. (1993) \\
\hline MSA & & 1990-1991 & SU & Jaffrezo et al. (1994) \\
\hline MSA & & 1991-1992 & ha & Minikin et al. (1998) \\
\hline MSA & & 1991-1992 & ha & Legrand and Pasteur (1998) \\
\hline MSA & & 1991-1995 & $\mathrm{du}$ & Minikin et al. (1998) \\
\hline MSA & & 1991-1995 & $\mathrm{nm}$ & Legrand et al. (1998) \\
\hline MSA & & 1991-1996 & $\mathrm{du}$ & Legrand and Pasteur (1998) \\
\hline MSA & & 1991-2001 & $\mathrm{du}$ & Jourdain and Legrand (2002) \\
\hline MSA & mean: $3.2 \mathrm{ng} / \mathrm{m}^{3}$ & Jun-Jul 1992 & $\mathrm{SU}$ & Bergin et al. (1994) \\
\hline MSA & mean: $5.6 \mathrm{ng} / \mathrm{m}^{3}$ & May-Jul 1993 & $\mathrm{SU}$ & Bergin et al. (1995) \\
\hline MSA & & Jan 1994 & $\mathrm{du}$ & Legrand et al. (1998) \\
\hline MSA & mean: $42.3 \mathrm{pmol} / \mathrm{mol}$ & Jan-Feb 1994 & $\mathrm{pa}$ & Berresheim et al. (1998) \\
\hline MSA & & 1998-1999 & $\mathrm{du}$ & $\begin{array}{l}\text { Jourdain and Legrand } \\
(2001) \text {,Legrand et al. (2001) }\end{array}$ \\
\hline MSA & mean: $12 \mathrm{ng} / \mathrm{m}^{3}$ & 1998-1999 & $\mathrm{sp}$ & Arimoto et al. (2001) \\
\hline MSA & & 1998,2000 & $\mathrm{sp}$ & Arimoto et al. (2004a) \\
\hline MSA & & Jan-Dec 2001 & ha & Rankin and Wolff (2003) \\
\hline MSA & mean: $50 \mathrm{ng} / \mathrm{m}^{3}$ & Apr-May 2001 & NA & Teinilä et al. (2003) \\
\hline \multicolumn{5}{|c|}{ Carbon } \\
\hline black carbon & $206 \ldots 295 \mathrm{ng} / \mathrm{m}^{3}$ & Mar 1983 & $\mathrm{BA}$ & Hansen and Rosen (1984) \\
\hline black carbon & & 1989-1990 & $\mathrm{AL}$ & Hopper et al. (1994b) \\
\hline black carbon & & $1989-2003$ & $\mathrm{AL}$ & Sharma et al. (2006) \\
\hline black carbon & & $1989-2003$ & $\mathrm{BA}$ & Sharma et al. (2006) \\
\hline black carbon & & $1992-1995$ & ha & Wolff and Cachier (1998) \\
\hline black carbon & & Feb-Mar 2000 & $\mathrm{AL}$ & Bottenheim et al. (2002b) \\
\hline oxalic acid & & $1991-1995$ & $\mathrm{du}$ & Legrand et al. (1998) \\
\hline oxalic acid & & $1991-2001$ & $\mathrm{du}$ & Jourdain and Legrand (2002) \\
\hline oxalic acid & & Jan 1994 & $\mathrm{du}$ & Legrand et al. (1998) \\
\hline oxalic acid & & Aug 1994 & SU & Dibb et al. (1996) \\
\hline oxalic acid & & Feb-May 2000 & $\mathrm{AL}$ & Narukawa et al. (2002) \\
\hline oxalic acid & mean: $8.6 \mathrm{ng} / \mathrm{m}^{3}$ & Apr-May 2001 & NA & Teinilä et al. (2003) \\
\hline oxalic acid & mean: $9.0 \mathrm{ng} / \mathrm{m}^{3}$ & Feb-Mar 2001 & NA & Teinilä et al. (2003) \\
\hline malonic acid & & Feb-May 2000 & $\mathrm{AL}$ & Narukawa et al. (2002) \\
\hline succinic acid & & Feb-May 2000 & $\mathrm{AL}$ & Narukawa et al. (2002) \\
\hline glutaric acid & & Feb-May 2000 & $\mathrm{AL}$ & Narukawa et al. (2002) \\
\hline adipic acid & & Feb-May 2000 & $\mathrm{AL}$ & Narukawa et al. (2002) \\
\hline pimelic acid & & Feb-May 2000 & $\mathrm{AL}$ & Narukawa et al. (2002) \\
\hline suberic acid & & Feb-May 2000 & $\mathrm{AL}$ & Narukawa et al. (2002) \\
\hline azelaic acid & & Feb-May 2000 & $\mathrm{AL}$ & Narukawa et al. (2002) \\
\hline sebacic acid & & Feb-May 2000 & $\mathrm{AL}$ & Narukawa et al. (2002) \\
\hline undecanedioic acid & & Feb-May 2000 & $\mathrm{AL}$ & Narukawa et al. (2002) \\
\hline methylmalonic acid & & Feb-May 2000 & $\mathrm{AL}$ & Narukawa et al. (2002) \\
\hline methylsuccinic acid & & Feb-May 2000 & $\mathrm{AL}$ & Narukawa et al. (2002) \\
\hline $\begin{array}{l}\text { 2-methylglutaric } \\
\text { acid }\end{array}$ & & Feb-May 2000 & $\mathrm{AL}$ & Narukawa et al. (2002) \\
\hline 4-ketopimelic acid & & Feb-May 2000 & $\mathrm{AL}$ & Narukawa et al. (2002) \\
\hline maleic acid & & Feb-May 2000 & $\mathrm{AL}$ & Narukawa et al. (2002) \\
\hline fumaric acid & & Feb-May 2000 & $\mathrm{AL}$ & Narukawa et al. (2002) \\
\hline methylmaleic acid & & Feb-May 2000 & $\mathrm{AL}$ & Narukawa et al. (2002) \\
\hline methylfumaric acid & & Feb-May 2000 & $\mathrm{AL}$ & Narukawa et al. (2002) \\
\hline phthalic acid & & Feb-May 2000 & $\mathrm{AL}$ & Narukawa et al. (2002) \\
\hline acetate & & Mar-May 1986 & $\mathrm{BA}$ & Li and Winchester (1989a,b) \\
\hline acetate & & Aug 1994 & SU & Dibb et al. (1996) \\
\hline acetate & mean: $0.16 \mathrm{nmol} / \mathrm{m}^{3}(\mathrm{STP})$ & Jun-Jul 1993 & SU & Dibb et al. (1994) \\
\hline formate & & Mar-May 1986 & $\mathrm{BA}$ & Li and Winchester $(1989 a, b)$ \\
\hline formate & & Aug 1994 & $\mathrm{SU}$ & Dibb et al. (1996) \\
\hline
\end{tabular}




\begin{tabular}{|c|c|c|c|c|}
\hline species & value & date & site & reference \\
\hline formate & mean: $1.05 \mathrm{nmol} / \mathrm{m}^{3}(\mathrm{STP})$ & Jun-Jul 1993 & SU & Dibb et al. (1994) \\
\hline organic acids & & $1987-1988$ & $\mathrm{AL}$ & Kawamura and Kasukabe (1996) \\
\hline propanoate & & Mar-May 1986 & $\mathrm{BA}$ & Li and Winchester (1989a,b) \\
\hline pyruvate & & Mar-May 1986 & $\mathrm{BA}$ & Li and Winchester $(1989 a, b)$ \\
\hline \multicolumn{5}{|c|}{ Metals } \\
\hline $\mathrm{Al}$ & $0.57 \mathrm{ng} / \mathrm{m}^{3}(\mathrm{STP})$ & Oct 1970 & sp & Zoller et al. (1974) \\
\hline $\mathrm{Al}$ & mean: $30 \mathrm{ng} / \mathrm{m}^{3}$ (win) & 1976-1978 & $\mathrm{BA}$ & Rahn and McCaffrey (1979) \\
\hline $\mathrm{Al}$ & & $1979-1980$ & $\mathrm{ARC}$ & Heidam (1985) \\
\hline $\mathrm{Al}$ & & 1979-1984 & $\mathrm{ARC}$ & Barrie and Hoff (1985) \\
\hline $\mathrm{Al}$ & & 1980-1986 & $\mathrm{AL}$ & Barrie and Barrie (1990) \\
\hline $\mathrm{Al}$ & & 1980-1995 & $\mathrm{AL}$ & Sirois and Barrie (1999) \\
\hline $\mathrm{Al}$ & & $1980-2000$ & $\mathrm{AL}$ & Gong and Barrie (2005) \\
\hline $\mathrm{Al}$ & & Apr-May 1980 & $\mathrm{MB}, \mathrm{IG}$ & Barrie et al. (1981) \\
\hline $\mathrm{Al}$ & & Aug-Sep 1983 & $\mathrm{ARC}$ & Pacyna and Ottar (1985) \\
\hline $\mathrm{Al}$ & & $1983-1986$ & $\mathrm{NA}, \mathrm{NO}$ & Maenhaut et al. (1989) \\
\hline $\mathrm{Al}$ & & 1984-1987 & $\mathrm{PF}$ & Sturges and Shaw (1993) \\
\hline $\mathrm{Al}$ & $15 \ldots 976 \mathrm{ng} / \mathrm{m}^{3}$ & spr 1988 & $\mathrm{AL}$ & Bottenheim et al. (1990) \\
\hline $\mathrm{Al}$ & mean: $4.2 \mathrm{ng} / \mathrm{m}^{3}$ & $1988-1989$ & DY & Davidson et al. (1993b,a) \\
\hline $\mathrm{Al}$ & mean: $5.5 \mathrm{ng} / \mathrm{m}^{3}$ & $1988-1989$ & DY & Davidson et al. (1993b,a) \\
\hline $\mathrm{Al}$ & $0.29 \ldots 260 \mathrm{ng} / \mathrm{m}^{3}$ & 1988-1989 & DY & Mosher et al. (1993) \\
\hline $\mathrm{Al}$ & $1.1 \ldots 48 \mathrm{ng} / \mathrm{m}^{3}$ & 1988-1989 & SU & Mosher et al. (1993) \\
\hline $\mathrm{Al}$ & & $1990-2001$ & $\mathrm{SN}$ & Heidam et al. (2004) \\
\hline $\mathrm{Al}$ & mean: $44.36 \mathrm{ng} / \mathrm{m}^{3}$ & $2002-2005$ & $\mathrm{NU}$ & Skov et al. (2006) \\
\hline As & & 1984-1987 & $\mathrm{PF}$ & Sturges and Shaw (1993) \\
\hline As & & 1990-2001 & $\mathrm{SN}$ & Heidam et al. (2004) \\
\hline As & mean: $0.02 \mathrm{ng} / \mathrm{m}^{3}$ & 2002-2005 & NU & Skov et al. (2006) \\
\hline $\mathrm{Ba}$ & & 1979-1984 & $\mathrm{ARC}$ & Barrie and Hoff (1985) \\
\hline $\mathrm{Ba}$ & & $1980-2000$ & $\mathrm{AL}$ & Gong and Barrie (2005) \\
\hline $\mathrm{Ba}$ & & 1983-1986 & $\mathrm{NA}, \mathrm{NO}$ & Maenhaut et al. (1989) \\
\hline $\mathrm{Ca}$ & $0.5 \mathrm{ng} / \mathrm{m}^{3}(\mathrm{STP})$ & Oct 1970 & $\mathrm{sp}$ & Zoller et al. (1974) \\
\hline $\mathrm{Ca}$ & mean: $55 \mathrm{ng} / \mathrm{m}^{3}$ (win) & 1976-1978 & $\mathrm{BA}$ & Rahn and McCaffrey (1979) \\
\hline $\mathrm{Ca}$ & & 1979-1980 & $\mathrm{ARC}$ & Heidam (1985) \\
\hline $\mathrm{Ca}$ & & 1979-1984 & $\mathrm{ARC}$ & Barrie and Hoff (1985) \\
\hline $\mathrm{Ca}$ & & 1980-1995 & $\mathrm{AL}$ & Sirois and Barrie (1999) \\
\hline $\mathrm{Ca}$ & & $1980-2000$ & $\mathrm{AL}$ & Gong and Barrie (2005) \\
\hline $\mathrm{Ca}$ & & Aug-Sep 1983 & $\mathrm{ARC}$ & Pacyna and Ottar (1985) \\
\hline $\mathrm{Ca}^{2+}$ & & $1983-1996$ & $\mathrm{~nm}$ & Wagenbach et al. (1998a) \\
\hline $\mathrm{Ca}$ & & $1983-1986$ & $\mathrm{NA}, \mathrm{NO}$ & Maenhaut et al. (1989) \\
\hline $\mathrm{Ca}$ & $19 \ldots 915 \mathrm{ng} / \mathrm{m}^{3}$ & spr 1988 & $\mathrm{AL}$ & Bottenheim et al. (1990) \\
\hline $\mathrm{Ca}$ & mean: $2.7 \mathrm{ng} / \mathrm{m}^{3}$ & $1988-1989$ & DY & Davidson et al. (1993b,a) \\
\hline $\mathrm{Ca}$ & mean: $4.2 \mathrm{ng} / \mathrm{m}^{3}$ & 1988-1989 & DY & Davidson et al. (1993b,a) \\
\hline $\mathrm{Ca}$ & $0.095 \ldots 170 \mathrm{ng} / \mathrm{m}^{3}$ & 1988-1989 & DY & Mosher et al. (1993) \\
\hline $\mathrm{Ca}$ & $0.5 \ldots 110 \mathrm{ng} / \mathrm{m}^{3}$ & 1988-1989 & $\mathrm{SU}$ & Mosher et al. (1993) \\
\hline $\mathrm{Ca}$ & & $1990-2001$ & $\mathrm{SN}$ & Heidam et al. (2004) \\
\hline $\mathrm{Ca}^{2+}$ & & 1991-1995 & $\mathrm{du}$ & Wagenbach et al. (1998a) \\
\hline $\mathrm{Ca}^{2+}$ & & 1991-1999 & $\mathrm{du}$ & Jourdain and Legrand (2002) \\
\hline $\mathrm{Ca}^{2+}$ & mean: $29 \mathrm{ng} / \mathrm{m}^{3}$ & May-Jul 1993 & SU & Bergin et al. (1995) \\
\hline $\mathrm{Ca}^{2+}$ & & Jan 1994 & $\mathrm{du}$ & Legrand et al. (1998) \\
\hline $\mathrm{Ca}^{2+}$ & & Aug 1994 & $\mathrm{SU}$ & Dibb et al. (1996) \\
\hline $\mathrm{nss}-\mathrm{Ca}^{2+}$ & mean: $32 \mathrm{ng} / \mathrm{m}^{3}$ & Apr-May 2001 & NA & Teinilä et al. (2003) \\
\hline $\mathrm{nss}-\mathrm{Ca}^{2+}$ & mean: $8.7 \mathrm{ng} / \mathrm{m}^{3}$ & Feb-Mar 2001 & NA & Teinilä et al. (2003) \\
\hline $\mathrm{Ca}$ & mean: $51.66 \mathrm{ng} / \mathrm{m}^{3}$ & $2002-2005$ & $\mathrm{NU}$ & Skov et al. (2006) \\
\hline $\mathrm{Cd}$ & mean: $0.37 \mathrm{ng} / \mathrm{m}^{3}$ (win) & $1976-1978$ & $\mathrm{BA}$ & Rahn and McCaffrey (1979) \\
\hline $\mathrm{Cd}$ & $\begin{array}{l}\text { annual mean: } 0.01 \ldots 0.03 \\
\mathrm{ng} / \mathrm{m}^{3}\end{array}$ & 1994-2002 & NA & Berg et al. (2004) \\
\hline $\mathrm{Co}$ & $\begin{array}{l}\text { annual mean: } 0.055 \ldots 0.14 \\
\mathrm{ng} / \mathrm{m}^{3}\end{array}$ & 1994-2002 & NA & Berg et al. (2004) \\
\hline $\mathrm{Cr}$ & $5.3 \mathrm{pg} / \mathrm{m}^{3}(\mathrm{STP})$ & Oct 1970 & $\mathrm{sp}$ & Zoller et al. (1974) \\
\hline $\mathrm{Cr}$ & & 1979-1980 & ARC & Heidam (1985) \\
\hline
\end{tabular}




\begin{tabular}{|c|c|c|c|c|}
\hline species & value & date & site & reference \\
\hline $\mathrm{Cr}$ & & 1979-1984 & $\mathrm{ARC}$ & Barrie and Hoff (1985) \\
\hline $\mathrm{Cr}$ & & Aug-Sep 1983 & $\mathrm{ARC}$ & Pacyna and Ottar (1985) \\
\hline $\mathrm{Cr}$ & & $1983-1986$ & $\mathrm{NA}, \mathrm{NO}$ & Maenhaut et al. (1989) \\
\hline $\mathrm{Cr}$ & $\begin{array}{l}\text { annual mean: } 0.04 \ldots 0.9 \\
\mathrm{ng} / \mathrm{m}^{3}\end{array}$ & 1994-2002 & NA & Berg et al. (2004) \\
\hline $\mathrm{Cr}$ & mean: $0.11 \mathrm{ng} / \mathrm{m}^{3}$ & 2002-2005 & $\mathrm{NU}$ & Skov et al. (2006) \\
\hline $\mathrm{Cu}$ & $36 \mathrm{pg} / \mathrm{m}^{3}(\mathrm{STP})$ & Oct 1970 & $\mathrm{sp}$ & Zoller et al. (1974) \\
\hline $\mathrm{Cu}$ & & 1979-1980 & $\mathrm{ARC}$ & Heidam (1985) \\
\hline $\mathrm{Cu}$ & & 1979-1984 & $\mathrm{ARC}$ & Barrie and Hoff (1985) \\
\hline $\mathrm{Cu}$ & & Apr-May 1980 & $\mathrm{MB}, \mathrm{IG}$ & Barrie et al. (1981) \\
\hline $\mathrm{Cu}$ & & $1980-1995$ & $\mathrm{AL}$ & Sirois and Barrie (1999) \\
\hline $\mathrm{Cu}$ & & $1980-2000$ & AL & Gong and Barrie (2005) \\
\hline $\mathrm{Cu}$ & & Aug-Sep 1983 & $\mathrm{ARC}$ & Pacyna and Ottar (1985) \\
\hline $\mathrm{Cu}$ & & $1983-1986$ & $\mathrm{NA}, \mathrm{NO}$ & Maenhaut et al. (1989) \\
\hline $\mathrm{Cu}$ & & $1990-2001$ & $\mathrm{SN}$ & Heidam et al. (2004) \\
\hline $\mathrm{Cu}$ & $\begin{array}{l}\text { annual mean: } 0.25 \ldots 0.41 \\
\mathrm{ng} / \mathrm{m}^{3}\end{array}$ & 1994-2002 & NA & Berg et al. (2004) \\
\hline $\mathrm{Cu}$ & mean: $0.36 \mathrm{ng} / \mathrm{m}^{3}$ & $2002-2005$ & $\mathrm{NU}$ & Skov et al. (2006) \\
\hline $\mathrm{Fe}$ & $0.84 \mathrm{ng} / \mathrm{m}^{3}(\mathrm{STP})$ & Oct 1970 & $\mathrm{sp}$ & Zoller et al. (1974) \\
\hline $\mathrm{Fe}$ & & $1979-1980$ & $\mathrm{ARC}$ & Heidam (1985) \\
\hline $\mathrm{Fe}$ & & 1979-1984 & $\mathrm{ARC}$ & Barrie and Hoff (1985) \\
\hline $\mathrm{Fe}$ & & $1980-2000$ & $\mathrm{AL}$ & Gong and Barrie (2005) \\
\hline $\mathrm{Fe}$ & & Aug-Sep 1983 & $\mathrm{ARC}$ & Pacyna and Ottar (1985) \\
\hline $\mathrm{Fe}$ & & $1983-1986$ & $\mathrm{NA}, \mathrm{NO}$ & Maenhaut et al. (1989) \\
\hline $\mathrm{Fe}$ & $0.1 \ldots 260 \mathrm{ng} / \mathrm{m}^{3}$ & $1988-1989$ & DY & Mosher et al. (1993) \\
\hline $\mathrm{Fe}$ & $0.45 \ldots 44 \mathrm{ng} / \mathrm{m}^{3}$ & 1988-1989 & $\mathrm{SU}$ & Mosher et al. (1993) \\
\hline $\mathrm{Fe}$ & & $1990-2001$ & $\mathrm{SN}$ & Heidam et al. (2004) \\
\hline $\mathrm{Fe}$ & mean: $33.42 \mathrm{ng} / \mathrm{m}^{3}$ & $2002-2005$ & $\mathrm{NU}$ & Skov et al. (2006) \\
\hline $\mathrm{Ga}$ & mean: $0.02 \mathrm{ng} / \mathrm{m}^{3}$ & $2002-2005$ & $\mathrm{NU}$ & Skov et al. (2006) \\
\hline $\mathrm{Hg}$ & mean: $<0.4 \mathrm{ng} / \mathrm{m}^{3}$ (win) & $1976-1978$ & $\mathrm{BA}$ & Rahn and McCaffrey (1979) \\
\hline $\mathrm{Hg}$ & mean: $1.44 \mathrm{pg} / \mathrm{m}^{3}$ & 1996-1997 & PS & Berg et al. (2001) \\
\hline $\mathrm{Hg}$ & mean: $2.67 \mathrm{pg} / \mathrm{m}^{3}$ & 1996-1997 & NA & Berg et al. (2001) \\
\hline $\mathrm{Hg}$ & & Apr-May 1998 & $\mathrm{AL}$ & Lu et al. (2001) \\
\hline $\mathrm{Hg}$ & & 2000 & $\mathrm{sp}$ & Arimoto et al. (2004b) \\
\hline $\mathrm{Hg}$ & & 2000 & NA & Berg et al. (2003) \\
\hline $\mathrm{Hg}$ & & Apr-Jun 2001 & $\mathrm{BA}$ & Lindberg et al. (2002) \\
\hline $\mathrm{Hg}$ & & Apr 2002 & $\mathrm{AL}$ & Steffen et al. (2003) \\
\hline $\mathrm{Hg}$ & & Apr-May 2002 & NA & Sommar et al. (2007) \\
\hline $\mathrm{Hg}$ & & Apr-May 2003 & NA & Sprovieri et al. $(2005 a, b)$ \\
\hline $\mathrm{Hg}$ & & Apr-May 2003 & NA & $\begin{array}{l}\text { Aspmo et al. (2005); Gauchard } \\
\text { et al. (2005) }\end{array}$ \\
\hline $\mathrm{K}$ & $0.3 \mathrm{ng} / \mathrm{m}^{3}(\mathrm{STP})$ & Oct 1970 & $\mathrm{sp}$ & Zoller et al. (1974) \\
\hline K & & $1979-1980$ & ARC & Heidam (1985) \\
\hline $\mathrm{K}^{+}$ & & 1980-1995 & $\mathrm{AL}$ & Sirois and Barrie (1999) \\
\hline $\mathrm{K}$ & & $1980-2000$ & $\mathrm{AL}$ & Gong and Barrie (2005) \\
\hline $\mathrm{K}$ & & Aug-Sep 1983 & $\mathrm{ARC}$ & Pacyna and Ottar (1985) \\
\hline $\mathrm{K}^{+}$ & & $1983-1996$ & $\mathrm{~nm}$ & Wagenbach et al. (1998a) \\
\hline K & & $1983-1986$ & $\mathrm{NA}, \mathrm{NO}$ & Maenhaut et al. (1989) \\
\hline $\mathrm{K}^{+}$ & & Mar-May 1986 & $\mathrm{BA}$ & $\mathrm{Li}$ and Winchester $(1989 \mathrm{a}, \mathrm{b})$ \\
\hline $\mathrm{K}^{+}$ & $3.6 \ldots 62.8 \mathrm{ng} / \mathrm{m}^{3}$ & spr 1988 & AL & Bottenheim et al. (1990) \\
\hline $\mathrm{K}^{+}$ & mean: $37 \mathrm{ng} / \mathrm{m}^{3}$ & May 1989 & $\mathrm{AL}$ & Kieser et al. (1993) \\
\hline K & & $1990-2001$ & $\mathrm{SN}$ & Heidam et al. (2004) \\
\hline $\mathrm{K}^{+}$ & & 1991-1995 & $\mathrm{du}$ & Wagenbach et al. (1998a) \\
\hline $\mathrm{K}^{+}$ & & 1991-1999 & $\mathrm{du}$ & Jourdain and Legrand (2002) \\
\hline $\mathrm{K}^{+}$ & mean: $3.4 \mathrm{ng} / \mathrm{m}^{3}$ & May-Jul 1993 & $\mathrm{SU}$ & Bergin et al. (1995) \\
\hline $\mathrm{K}^{+}$ & & Jan 1994 & $\mathrm{du}$ & Legrand et al. (1998) \\
\hline $\mathrm{K}^{+}$ & & Aug 1994 & SU & Dibb et al. (1996) \\
\hline $\mathrm{nss}-\mathrm{K}^{+}$ & mean: $6.0 \mathrm{ng} / \mathrm{m}^{3}$ & Apr-May 2001 & NA & Teinilä et al. (2003) \\
\hline $\mathrm{nss}-\mathrm{K}^{+}$ & mean: $8.8 \mathrm{ng} / \mathrm{m}^{3}$ & Feb-Mar 2001 & NA & Teinilä et al. (2003) \\
\hline $\mathrm{K}$ & mean: $42.89 \mathrm{ng} / \mathrm{m}^{3}$ & $2002-2005$ & $\mathrm{NU}$ & Skov et al. (2006) \\
\hline $\mathrm{Mg}$ & $1.0 \mathrm{ng} / \mathrm{m}^{3}(\mathrm{STP})$ & Oct 1970 & $\mathrm{sp}$ & Zoller et al. (1974) \\
\hline
\end{tabular}




\begin{tabular}{|c|c|c|c|c|}
\hline species & value & date & site & reference \\
\hline $\mathrm{Mg}$ & mean: $160 \mathrm{ng} / \mathrm{m}^{3}$ (win) & $1976-1978$ & $\mathrm{BA}$ & Rahn and McCaffrey (1979) \\
\hline $\mathrm{Mg}$ & & $1979-1984$ & $\mathrm{ARC}$ & Barrie and Hoff (1985) \\
\hline $\mathrm{Mg}$ & & $1980-1995$ & $\mathrm{AL}$ & Sirois and Barrie (1999) \\
\hline $\mathrm{Mg}$ & & $1980-2000$ & $\mathrm{AL}$ & Gong and Barrie (2005) \\
\hline $\mathrm{Mg}$ & & $1983-1986$ & $\mathrm{NA}, \mathrm{NO}$ & Maenhaut et al. (1989) \\
\hline $\mathrm{Mg}^{2+}$ & & $1983-1996$ & $\mathrm{~nm}$ & Wagenbach et al. (1998a) \\
\hline $\mathrm{Mg}$ & $13 \ldots 262 \mathrm{ng} / \mathrm{m}^{3}$ & spr 1988 & $\mathrm{AL}$ & Bottenheim et al. (1990) \\
\hline $\mathrm{Mg}$ & $0.69 \ldots 92 \mathrm{ng} / \mathrm{m}^{3}$ & $1988-1989$ & DY & Mosher et al. (1993) \\
\hline $\mathrm{Mg}$ & $2.5 \ldots 27 \mathrm{ng} / \mathrm{m}^{3}$ & 1988-1989 & $\mathrm{SU}$ & Mosher et al. (1993) \\
\hline $\mathrm{Mg}^{2+}$ & & 1991-1993 & ha & Wagenbach et al. (1998a) \\
\hline $\mathrm{Mg}^{2+}$ & & 1991-1995 & $\mathrm{du}$ & Wagenbach et al. (1998a) \\
\hline $\mathrm{Mg}^{2+}$ & mean: $6.8 \mathrm{ng} / \mathrm{m}^{3}$ & May-Jul 1993 & $\mathrm{SU}$ & Bergin et al. (1995) \\
\hline $\mathrm{Mg}^{2+}$ & & Jan 1994 & $\mathrm{du}$ & Legrand et al. (1998) \\
\hline $\mathrm{Mg}^{2+}$ & & Aug 1994 & $\mathrm{SU}$ & Dibb et al. (1996) \\
\hline $\mathrm{nss}-\mathrm{Mg}^{2+}$ & mean: $7.3 \mathrm{ng} / \mathrm{m}^{3}$ & Apr-May 2001 & NA & Teinilä et al. (2003) \\
\hline $\mathrm{nss}-\mathrm{Mg}^{2+}$ & mean: $7.7 \mathrm{ng} / \mathrm{m}^{3}$ & Feb-Mar 2001 & NA & Teinilä et al. (2003) \\
\hline Mn & $10.3 \mathrm{pg} / \mathrm{m}^{3}(\mathrm{STP})$ & Oct 1970 & $\mathrm{sp}$ & Zoller et al. (1974) \\
\hline $\mathrm{Mn}$ & mean: $1.12 \mathrm{ng} / \mathrm{m}^{3}$ (win) & $1976-1978$ & $\mathrm{BA}$ & Rahn and McCaffrey (1979) \\
\hline $\mathrm{Mn}$ & & $1979-1980$ & $\mathrm{ARC}$ & Heidam (1985) \\
\hline $\mathrm{Mn}$ & & $1979-1984$ & $\mathrm{ARC}$ & Barrie and Hoff (1985) \\
\hline $\mathrm{Mn}$ & & Apr-May 1980 & $\mathrm{MB}, \mathrm{IG}$ & Barrie et al. (1981) \\
\hline Mn & & 1980-1995 & $\mathrm{AL}$ & Sirois and Barrie (1999) \\
\hline $\mathrm{Mn}$ & & $1980-2000$ & $\mathrm{AL}$ & Gong and Barrie (2005) \\
\hline $\mathrm{Mn}$ & & Aug-Sep 1983 & $\mathrm{ARC}$ & Pacyna and Ottar (1985) \\
\hline $\mathrm{Mn}$ & & $1983-1986$ & $\mathrm{NA}, \mathrm{NO}$ & Maenhaut et al. (1989) \\
\hline Mn & $0.19 \ldots 6.7 \mathrm{ng} / \mathrm{m}^{3}$ & spr 1988 & $\mathrm{AL}$ & Bottenheim et al. (1990) \\
\hline $\mathrm{Mn}$ & $0.004 \ldots 3.3 \mathrm{ng} / \mathrm{m}^{3}$ & 1988-1989 & DY & Mosher et al. (1993) \\
\hline $\mathrm{Mn}$ & $0.052 \ldots 0.84 \mathrm{ng} / \mathrm{m}^{3}$ & 1988-1989 & SU & Mosher et al. (1993) \\
\hline $\mathrm{Mn}$ & & $1990-2001$ & $\mathrm{SN}$ & Heidam et al. (2004) \\
\hline $\mathrm{Mn}$ & $\begin{array}{l}\text { annual mean: } 0.24 \ldots 0.57 \\
\mathrm{ng} / \mathrm{m}^{3}\end{array}$ & $1994-2002$ & NA & Berg et al. (2004) \\
\hline Mn & mean: $0.60 \mathrm{ng} / \mathrm{m}^{3}$ & $2002-2005$ & NU & Skov et al. (2006) \\
\hline $\mathrm{Na}$ & $7.2 \mathrm{ng} / \mathrm{m}^{3}(\mathrm{STP})$ & Oct 1970 & $\mathrm{sp}$ & Zoller et al. (1974) \\
\hline $\mathrm{Na}$ & mean: $770 \mathrm{ng} / \mathrm{m}^{3}$ (win) & $1976-1978$ & $\mathrm{BA}$ & Rahn and McCaffrey (1979) \\
\hline $\mathrm{Na}$ & & $1976-1980$ & $\mathrm{BA}$ & Berg et al. (1983) \\
\hline $\mathrm{Na}$ & & $1979-1984$ & $\mathrm{ARC}$ & Barrie and Hoff (1985) \\
\hline $\mathrm{Na}$ & & $1980-1986$ & $\mathrm{AL}$ & Barrie and Barrie (1990) \\
\hline $\mathrm{Na}^{+}$ & & 1980-1995 & $\mathrm{AL}$ & Sirois and Barrie (1999) \\
\hline $\mathrm{Na}$ & & $1980-2000$ & $\mathrm{AL}$ & Gong and Barrie (2005) \\
\hline $\mathrm{Na}^{+}$ & & Apr-May 1980 & $\mathrm{MB}, \mathrm{IG}$ & Barrie et al. (1981) \\
\hline $\mathrm{Na}^{+}$ & $74 \ldots 289 \mathrm{ng} / \mathrm{m}^{3}$ & Feb 1982 & IG & Hoff et al. (1983) \\
\hline $\mathrm{Na}$ & $14 \ldots 188 \mathrm{ng} / \mathrm{m}^{3}$ & Feb 1982 & IG & Hoff et al. (1983) \\
\hline $\mathrm{Na}$ & $<40 \ldots 236 \mathrm{ng} / \mathrm{m}^{3}$ & spr 1983 & $\mathrm{ARC}$ & Cahill and Eldred (1984) \\
\hline $\mathrm{Na}$ & & $1983-1986$ & $\mathrm{NA}, \mathrm{NO}$ & Maenhaut et al. (1989) \\
\hline $\mathrm{Na}^{+}$ & & $1983-1996$ & $\mathrm{~nm}$ & Wagenbach et al. (1998a) \\
\hline $\mathrm{Na}$ & & $1984-1987$ & $\mathrm{PF}$ & Sturges and Shaw (1993) \\
\hline $\mathrm{Na}^{+}$ & & Mar-May 1986 & $\mathrm{BA}$ & $\mathrm{Li}$ and Winchester $(1989 \mathrm{a}, \mathrm{b})$ \\
\hline $\mathrm{Na}$ & $24 \ldots 1240 \mathrm{ng} / \mathrm{m}^{3}$ & spr 1988 & $\mathrm{AL}$ & Bottenheim et al. (1990) \\
\hline $\mathrm{Na}$ & $0.1 \ldots 300 \mathrm{ng} / \mathrm{m}^{3}$ & $1988-1989$ & DY & Mosher et al. (1993) \\
\hline $\mathrm{Na}$ & $0.52 \ldots 17.4 \mathrm{ng} / \mathrm{m}^{3}$ & 1988-1989 & SU & Mosher et al. (1993) \\
\hline $\mathrm{Na}$ & mean: $8.0 \mathrm{ng} / \mathrm{m}^{3}$ & 1988-1989 & DY & Davidson et al. (1993b,a) \\
\hline $\mathrm{Na}^{+}$ & mean: $117 \mathrm{ng} / \mathrm{m}^{3}$ & May 1989 & $\mathrm{AL}$ & Kieser et al. (1993) \\
\hline $\mathrm{Na}^{+}$ & & 1991-1993 & ha & Wagenbach et al. (1998a) \\
\hline $\mathrm{Na}^{+}$ & & 1991-1995 & $\mathrm{du}$ & Wagenbach et al. (1998a) \\
\hline $\mathrm{Na}^{+}$ & & 1991-1999 & $\mathrm{du}$ & Jourdain and Legrand (2002) \\
\hline $\mathrm{Na}^{+}$ & mean: $14 \mathrm{ng} / \mathrm{m}^{3}$ & May-Jul 1993 & $\mathrm{SU}$ & Bergin et al. (1995) \\
\hline $\mathrm{Na}^{+}$ & & 1996-1999 & NA & Hara et al. (2002a) \\
\hline $\mathrm{Na}^{+}$ & $0 \ldots 1062.0 \mathrm{pmol} / \mathrm{mol}$ & 1997-1999 (spr) & NA & Beine et al. (2001) \\
\hline $\mathrm{Na}$ & mean: $45 \mathrm{ng} / \mathrm{m}^{3}$ & 1998-1999 & $\mathrm{sp}$ & Arimoto et al. (2001) \\
\hline $\mathrm{Na}^{+}$ & & 1998,2000 & sp & Arimoto et al. (2004a) \\
\hline $\mathrm{Na}^{+}$ & mean: $339.3 \mathrm{ng} / \mathrm{m}^{3}$ & Apr-May 2000 & $\mathrm{AL}$ & Ianniello et al. (2002) \\
\hline
\end{tabular}




\begin{tabular}{|c|c|c|c|c|}
\hline species & value & date & site & reference \\
\hline $\mathrm{Na}^{+}$ & mean: $69.1 \mathrm{ng} / \mathrm{m}^{3}$ & Feb 2000 & $\mathrm{AL}$ & Ianniello et al. (2002) \\
\hline $\mathrm{Na}$ & & Mar-Apr 2000 & NA & Hara et al. (2002b) \\
\hline $\mathrm{Na}$ & & Jan-Dec 2001 & ha & Rankin and Wolff (2003) \\
\hline $\mathrm{Na}^{+}$ & mean: $312 \mathrm{ng} / \mathrm{m}^{3}$ & Feb-Mar 2001 & NA & Teinilä et al. (2003) \\
\hline $\mathrm{Na}^{+}$ & mean: $261 \mathrm{ng} / \mathrm{m}^{3}$ & Apr-May 2001 & NA & Teinilä et al. (2003) \\
\hline $\mathrm{Ni}$ & & $1979-1980$ & $\mathrm{ARC}$ & Heidam (1985) \\
\hline $\mathrm{Ni}$ & & $1979-1984$ & $\mathrm{ARC}$ & Barrie and Hoff (1985) \\
\hline $\mathrm{Ni}$ & & Apr-May 1980 & $\mathrm{MB}, \mathrm{IG}$ & Barrie et al. (1981) \\
\hline $\mathrm{Ni}$ & & $1980-2000$ & $\mathrm{AL}$ & Gong and Barrie (2005) \\
\hline $\mathrm{Ni}$ & & Aug-Sep 1983 & $\mathrm{ARC}$ & Pacyna and Ottar (1985) \\
\hline $\mathrm{Ni}$ & & $1983-1986$ & $\mathrm{NA}, \mathrm{NO}$ & Maenhaut et al. (1989) \\
\hline $\mathrm{Ni}$ & & $1990-2001$ & $\mathrm{SN}$ & Heidam et al. (2004) \\
\hline $\mathrm{Ni}$ & $\begin{array}{l}\text { annual mean: } 0.07 \ldots 0.19 \\
\mathrm{ng} / \mathrm{m}^{3}\end{array}$ & $1994-2002$ & NA & Berg et al. (2004) \\
\hline $\mathrm{Ni}$ & mean: $0.17 \mathrm{ng} / \mathrm{m}^{3}$ & 2002-2005 & $\mathrm{NU}$ & Skov et al. (2006) \\
\hline $\mathrm{Pb}$ & $0.63 \mathrm{ng} / \mathrm{m}^{3}(\mathrm{STP})$ & Oct 1970 & $\mathrm{sp}$ & Zoller et al. (1974) \\
\hline $\mathrm{Pb}$ & & $1979-1980$ & $\mathrm{ARC}$ & Heidam (1985) \\
\hline $\mathrm{Pb}$ & & $1979-1984$ & $\mathrm{ARC}$ & Barrie and Hoff (1985) \\
\hline $\mathrm{Pb}$ & & Apr-May 1980 & $\mathrm{MB}, \mathrm{IG}$ & Barrie et al. (1981) \\
\hline $\mathrm{Pb}$ & & $1980-1986$ & $\mathrm{AL}$ & Barrie and Barrie (1990) \\
\hline $\mathrm{Pb}$ & & 1980-1995 & $\mathrm{AL}$ & Sirois and Barrie (1999) \\
\hline $\mathrm{Pb}$ & & $1980-2000$ & $\mathrm{AL}$ & Gong and Barrie (2005) \\
\hline $\mathrm{Pb}$ & & Aug-Sep 1983 & $\mathrm{ARC}$ & Pacyna and Ottar (1985) \\
\hline $\mathrm{Pb}$ & & $1983-1986$ & $\mathrm{NA}, \mathrm{NO}$ & Maenhaut et al. (1989) \\
\hline $\mathrm{Pb}$ & & Feb-Mar 1990 & $\mathrm{BA}$ & Sturges et al. (1993a) \\
\hline $\mathrm{Pb}$ & & $1990-2001$ & $\mathrm{SN}$ & Heidam et al. (2004) \\
\hline $\mathrm{Pb}$ & $\begin{array}{l}\text { annual mean: } 0.48 \ldots 0.83 \\
\mathrm{ng} / \mathrm{m}^{3}\end{array}$ & 1994-2002 & NA & Berg et al. (2004) \\
\hline $\mathrm{Pb}$ & & 2000 & $\mathrm{sp}$ & Arimoto et al. (2004b) \\
\hline $\mathrm{Pb}$ & mean: $0.48 \mathrm{ng} / \mathrm{m}^{3}$ & $2002-2005$ & NU & Skov et al. (2006) \\
\hline $\mathrm{Rb}$ & mean: $0.11 \mathrm{ng} / \mathrm{m}^{3}$ & $2002-2005$ & NU & Skov et al. (2006) \\
\hline Se & & $1983-1986$ & $\mathrm{NA}, \mathrm{NO}$ & Maenhaut et al. (1989) \\
\hline $\mathrm{Se}$ & $\begin{array}{l}\text { monthly mean: } 0.03 \ldots 0.08 \\
\mathrm{ng} / \mathrm{m}^{3}\end{array}$ & 1984-1987 & $\mathrm{PF}$ & Sturges and Shaw (1993) \\
\hline $\mathrm{Se}$ & mean: $0.05 \mathrm{ng} / \mathrm{m}^{3}$ & 2002-2005 & $\mathrm{NU}$ & Skov et al. (2006) \\
\hline $\mathrm{Si}$ & & $1990-2001$ & SN & Heidam et al. (2004) \\
\hline $\mathrm{Si}$ & mean: $143.46 \mathrm{ng} / \mathrm{m}^{3}$ & $2002-2005$ & $\mathrm{NU}$ & Skov et al. (2006) \\
\hline $\mathrm{Sr}$ & & $1979-1980$ & ARC & Heidam (1985) \\
\hline $\mathrm{Sr}$ & & 1979-1984 & $\mathrm{ARC}$ & Barrie and Hoff (1985) \\
\hline $\mathrm{Sr}$ & & $1983-1986$ & $\mathrm{NA}, \mathrm{NO}$ & Maenhaut et al. (1989) \\
\hline $\mathrm{Sr}$ & & $1990-2001$ & $\mathrm{SN}$ & Heidam et al. (2004) \\
\hline $\mathrm{Sr}$ & mean: $0.79 \mathrm{ng} / \mathrm{m}^{3}$ & $2002-2005$ & $\mathrm{NU}$ & Skov et al. (2006) \\
\hline $\mathrm{Ti}$ & & 1979-1984 & $\mathrm{ARC}$ & Barrie and Hoff (1985) \\
\hline $\mathrm{Ti}$ & & $1980-2000$ & $\mathrm{AL}$ & Gong and Barrie (2005) \\
\hline $\mathrm{Ti}$ & & Aug-Sep 1983 & $\mathrm{ARC}$ & Pacyna and Ottar (1985) \\
\hline $\mathrm{Ti}$ & & $1983-1986$ & $\mathrm{NA}, \mathrm{NO}$ & Maenhaut et al. (1989) \\
\hline $\mathrm{Ti}$ & & $1990-2001$ & $\mathrm{SN}$ & Heidam et al. (2004) \\
\hline $\mathrm{Ti}$ & mean: $3.09 \mathrm{ng} / \mathrm{m}^{3}$ & $2002-2005$ & $\mathrm{NU}$ & Skov et al. (2006) \\
\hline $\mathrm{V}$ & $1.5 \mathrm{pg} / \mathrm{m}^{3}(\mathrm{STP})$ & Oct 1970 & $\mathrm{sp}$ & Zoller et al. (1974) \\
\hline $\mathrm{V}$ & mean: $0.65 \mathrm{ng} / \mathrm{m}^{3}$ (win) & 1976-1978 & $\mathrm{BA}$ & Rahn and McCaffrey (1979) \\
\hline $\mathrm{V}$ & & 1979-1984 & $\mathrm{ARC}$ & Barrie and Hoff (1985) \\
\hline $\mathrm{V}$ & & Apr-May 1980 & $\mathrm{MB}, \mathrm{IG}$ & Barrie et al. (1981) \\
\hline $\mathrm{V}$ & & $1980-1995$ & $\mathrm{AL}$ & Sirois and Barrie (1999) \\
\hline $\mathrm{V}$ & & $1980-2000$ & $\mathrm{AL}$ & Gong and Barrie (2005) \\
\hline $\mathrm{V}$ & & Aug-Sep 1983 & $\mathrm{ARC}$ & Pacyna and Ottar (1985) \\
\hline $\mathrm{V}$ & & $1983-1986$ & $\mathrm{NA}, \mathrm{NO}$ & Maenhaut et al. (1989) \\
\hline $\mathrm{V}$ & & $1984-1987$ & $\mathrm{PF}$ & Sturges and Shaw (1993) \\
\hline $\mathrm{V}$ & $0.05 \ldots 1.72 \mathrm{ng} / \mathrm{m}^{3}$ & spr 1988 & $\mathrm{AL}$ & Bottenheim et al. (1990) \\
\hline $\mathrm{V}$ & $0.014 \ldots 5.5 \mathrm{ng} / \mathrm{m}^{3}$ & $1988-1989$ & DY & Mosher et al. (1993) \\
\hline $\mathrm{V}$ & $0.006 \ldots .25 \mathrm{ng} / \mathrm{m}^{3}$ & 1988-1989 & SU & Mosher et al. (1993) \\
\hline
\end{tabular}




\begin{tabular}{|c|c|c|c|c|}
\hline species & value & date & site & reference \\
\hline $\mathrm{V}$ & & $1990-2001$ & SN & Heidam et al. (2004) \\
\hline $\mathrm{V}$ & $\begin{array}{l}\text { annual mean: } 0.07 \ldots 0.20 \\
\mathrm{ng} / \mathrm{m}^{3}\end{array}$ & 1994-2002 & NA & Berg et al. (2004) \\
\hline $\mathrm{V}$ & mean: $0.17 \mathrm{ng} / \mathrm{m}^{3}$ & $2002-2005$ & $\mathrm{NU}$ & Skov et al. (2006) \\
\hline $\mathrm{Zn}$ & $30 \mathrm{pg} / \mathrm{m}^{3}(\mathrm{STP})$ & Oct 1970 & $\mathrm{sp}$ & Zoller et al. (1974) \\
\hline $\mathrm{Zn}$ & mean: $14.8 \mathrm{ng} / \mathrm{m}^{3}$ (win) & 1976-1978 & $\mathrm{BA}$ & Rahn and McCaffrey (1979) \\
\hline $\mathrm{Zn}$ & & $1979-1980$ & $\mathrm{ARC}$ & Heidam (1985) \\
\hline $\mathrm{Zn}$ & & $1979-1984$ & $\mathrm{ARC}$ & Barrie and Hoff (1985) \\
\hline $\mathrm{Zn}$ & & Apr-May 1980 & $\mathrm{MB}, \mathrm{IG}$ & Barrie et al. (1981) \\
\hline $\mathrm{Zn}$ & & 1980-1995 & $\mathrm{AL}$ & Sirois and Barrie (1999) \\
\hline $\mathrm{Zn}$ & & $1980-2000$ & $\mathrm{AL}$ & Gong and Barrie (2005) \\
\hline $\mathrm{Zn}$ & & Aug-Sep 1983 & $\mathrm{ARC}$ & Pacyna and Ottar (1985) \\
\hline $\mathrm{Zn}$ & & $1983-1986$ & $\mathrm{NA}, \mathrm{NO}$ & Maenhaut et al. (1989) \\
\hline $\mathrm{Zn}$ & mean: $0.63 \mathrm{ng} / \mathrm{m}^{3}$ & $1988-1989$ & DY & Davidson et al. (1993b,a) \\
\hline $\mathrm{Zn}$ & & $1990-2001$ & $\mathrm{SN}$ & Heidam et al. (2004) \\
\hline $\mathrm{Zn}$ & $\begin{array}{l}\text { annual mean: } 1.2 \ldots 1.9 \\
\mathrm{ng} / \mathrm{m}^{3}\end{array}$ & 1994-2002 & NA & Berg et al. (2004) \\
\hline $\mathrm{Zn}$ & mean: $2.62 \mathrm{ng} / \mathrm{m}^{3}$ & $2002-2005$ & $\mathrm{NU}$ & Skov et al. (2006) \\
\hline $\mathrm{Zr}$ & mean: $0.10 \mathrm{ng} / \mathrm{m}^{3}$ & $2002-2005$ & $\mathrm{NU}$ & Skov et al. (2006) \\
\hline
\end{tabular}




\section{References}

Albrecht, T., Notholt, J., Wolke, R., Solberg, S., Dye, C., and Malberg, H.: Variations of $\mathrm{CH}_{2} \mathrm{O}$ and $\mathrm{C}_{2} \mathrm{H}_{2}$ determined from ground-based FTIR measurements and comparison with model results, Adv. Space Res., 29, 1713-1718, 2002 .

Allegrini, I., Montagnoli, M., and Sparapani, R.: Evaluation of gas phase and particulate components relevant to polar tropospheric processes, Int. J. Environ. Anal. Chem., 55, 267-283, 1994.

Allegrini, I., Ianniello, A., Montagnoli, M., Sparapani, R., and Mazziotti Gomez de Teran, C.: Carbon-coated annular denuders and ion chromatographic measurements for the determination of nitrogen-containing species $\left(\mathrm{NO}_{2}\right.$ and $\left.\mathrm{NO}_{\mathrm{y}}\right)$ in remote atmospheres, J. Chromatogr. A, 846, 265-268, 1999.

Amoroso, A., Beine, H. J., Sparapani, R., and Nardino, M.: Observation of coinciding Arctic boundary layer ozone depletion and snow surface emissions of nitrous acid, Atmos. Environ., 40, 1949-1956, 2005.

Anlauf, K. G., Mickle, R. E., and Trivett, N. B. A.: Measurement of ozone during Polar Sunrise Experiment 1992, J. Geophys. Res., 99D, 25 345-25 353, 1994.

Arimoto, R., Nottingham, A. S., Webb, J., Schloesslin, C. A., and Davis, D. D.: Non-sea salt sulfate and other aerosol constituents at the South Pole during ISCAT, Geophys. Res. Lett., 28, 3645-3648, 2001.

Arimoto, R., Hogan, A., Grube, P., Davis, D., Webb, J., Schloesslin, C., Sage, S., and Raccah, F.: Major ions and radionuclides in aerosol particles from the South Pole during ISCAT-2000, Atmos. Environ., 38, 5473-5484, 2004a.

Arimoto, R., Schloesslin, C., Davis, D., Hogan, A., Grube, P., Fitzgerald, W., and Lamborg, C.: Lead and mercury in aerosol particles collected over the South Pole during ISCAT-2000, Atmos. Environ., 38, 5485-5491, 2004b.

Ariya, P. A., Jobson, B. T., Sander, R., Niki, H., Harris, G. W., Anlauf, K. G., and Hopper, J. F.: Measurements of $\mathrm{C}_{2}$ $\mathrm{C}_{7}$ hydrocarbons during the polar sunrise experiment 1994: Further evidence for halogen chemistry in the troposphere, J. Geophys. Res., 103D, 13 169-13 180, 1998.

Ariya, P. A., Niki, H., Harris, G. W., Anlauf, K. G., and Worthy, D. E. J.: Polar sunrise experiment 1995: Hydrocarbon measurements and tropospheric $\mathrm{Cl}$ and Br-atoms chemistry, Atmos. Environ., 33, 931-938, 1999.

Aspmo, K., Gauchard, P.-A., Steffen, A., Temme, C., Berg, T., Bahlmann, E., Banic, C., Dommergue, A., Ebinghaus, R., Ferrari, C., Pirrone, N., Sprovieri, F., and Wibetoe, G.: Measurements of atmospheric mercury species during an international study of mercury depletion events at $\mathrm{Ny}-$ Ålesund, Svalbard, spring 2003. How reproducible are our present methods?, Atmos. Environ., 39, 7607-7619, 2005.

Bales, R. C., Losleben, M. V., McConnell, J. R., Fuhrer, K., and Neftel, A.: $\mathrm{H}_{2} \mathrm{O}_{2}$ in snow, air and open pore space in firn at Summit, Greenland, Geophys. Res. Lett., 22, 1261-1264, 1995a.
Bales, R. C., McConnell, J. R., Losleben, M. V., Conklin, M. H., Fuhrer, K., Neftel, A., Dibb, J. E., Kahl, J. D. W., and Stearns, C. R.: Diel variations of $\mathrm{H}_{2} \mathrm{O}_{2}$ in Greenland: A discussion of the cause and effect relationship, J. Geophys. Res., 100D, 18661-18668, 1995b.

Banic, C. M., Beauchamp, S. T., Tordon, R. J., Schroeder, W. H., Steffen, A., Anlauf, K. A., and Wong, H. K. T.: Vertical distribution of gaseous elemental mercury in Canada, J. Geophys. Res., 108D, 4264, doi:10.1029/2002JD002116, 2003.

Barrie, L. A. and Barrie, M. J.: Chemical components of lower tropospheric aerosols in the high Arctic: Six years of observations, J. Atmos. Chem., 11, 211-226, 1990.

Barrie, L. A. and Delmas, R. J.: Polar atmosphere and snow chemistry, in: Global Atmospheric-Biospheric Chemistry, edited by Prinn, R. G., pp. 149-164, Plenum Press, NY, 1994.

Barrie, L. A. and Hoff, R. M.: The oxidation rate and residence time of sulphur dioxide in the Arctic atmosphere, Atmos. Environ., 18, 2711-2722, 1984.

Barrie, L. A. and Hoff, R. M.: Five years of air chemistry observations in the Canadian Arctic, Atmos. Environ., 19, 1995-2010, 1985.

Barrie, L. A., Hoff, R. M., and Daggupaty, S. M.: The influence of mid-latitudinal pollution sources on haze in the Canadian Arctic, Atmos. Environ., 15, 1407-1419, 1981.

Barrie, L. A., Li, S.-M., Toom, D. L., Landsberger, S., and Sturges, W.: Lower tropospheric measurements of halogens, nitrates, and sulphur oxides during Polar Sunrise Experiment 1992, J. Geophys. Res., 99D, 25 453-25 467, 1994a.

Barrie, L. A., Staebler, R., Toom, D., Georgi, B., den Hartog, G., Landsberger, S., and Wu, D.: Arctic aerosol sizesegregated chemical observations in relation to ozone depletion during Polar Sunrise Experiment 1992, J. Geophys. Res., 99D, 25 439-25 451, 1994 b.

Beine, H. J.: Measurements of CO in the high Arctic, Chemosphere - Global Change Science, 1, 145-151, 1999.

Beine, H. J. and Krognes, T.: The seasonal cycle of peroxyacetyl nitrate (PAN) in the Arctic, Atmos. Environ., 34, 933-940, 2000.

Beine, H. J., Engardt, M., Jaffe, D. A., Hov, Ø., Holmén, K., and Stordal, F.: Measurements of $\mathrm{NO}_{\mathrm{x}}$ and aerosol particles at the $\mathrm{Ny}$-Ålesund Zeppelin mountain-station on Svalbard: Influence of local and regional pollution sources, Atmos. Environ., 30, 1067-1079, 1996a.

Beine, H. J., Jaffe, D. A., Blake, D. R., Atlas, E., and Harris, J.: Measurements of PAN, alkyl nitrates, ozone and hydrocarbons during spring in interior Alaska, J. Geophys. Res., 101D, 12 613-12 619, 1996b.

Beine, H. J., Jaffe, D. A., Herring, J. A., Kelley, J. A., Krognes, T., and Stordal, F.: High-latitude springtime photochemistry. Part I: $\mathrm{NO}_{\mathrm{x}}, \mathrm{PAN}$ and ozone relationship, J. Atmos. Chem., 27, 127-153, 1997a. 
Beine, H. J., Jaffe, D. A., Stordal, F., Engardt, M., Solberg, S., Schmidbauer, N., and Holmen, K.: $\mathrm{NO}_{\mathrm{x}}$ during ozone depletion events in the Arctic troposphere at $\mathrm{Ny}$-Ålesund, Svalbard, Tellus, 49B, 556-565, 1997b.

Beine, H. J., Allegrini, I., Sparapani, R., Ianniello., A., and Valentini, F.: Three years of springtime trace gas and particle measurement at Ny-Ålesund, Svalbard, Atmos. Environ., 35, 3645-3658, 2001.

Beine, H. J., Honrath, R. E., Dominé, F., Simpson, W. R., and Fuentes, J. D.: $\mathrm{NO}_{\mathrm{x}}$ during background and ozone depletion periods at Alert: Fluxes above the snow surface, J. Geophys. Res., 107D, 4584, doi:10.1029/2002JD002082, 2002.

Beine, H. J., Dominé, F., Ianniello, A., Nardino, M., Allegrini, I., Teinilä, K., and Hillamo, R.: Fluxes of nitrates between snow surfaces and the atmosphere in the European high Arctic, Atmos. Chem. Phys., 3, 335-346, 2003.

Beine, H. J., Amoroso, A., Dominë, F., King, M., Nardino, M., and A. Ianniello, J. L. F.: Surprisingly small HONO emissions from snow surfaces at Browning Pass, Antarctica, Atmos. Chem. Phys., 6, 2569-2580, 2006.

Berg, T., Bartnicki, J., Munthe, J., Lattila, H., Hrehoruk, J., and Mazur, A.: Atmospheric mercury species in the European Arctic: Measurements and modelling, Atmos. Environ., 35, 2569-2582, 2001.

Berg, T., Sekkesæter, S., Steinnes, E., Valdal, A.-K., and Wibetoe, G.: Springtime depletion of mercury in the European Arctic as observed at Svalbard, Sci. Total Environ., 304, 4351, 2003.

Berg, T., Kallenborn, R., and Manø, S.: Temporal trends in atmospheric heavy metal and organochlorine concentrations at Zeppelin, Svalbard, Arct. Antarct. Alp. Res., 36, 284-291, 2004.

Berg, W. W., Sperry, P. D., Rahn, K. A., and Gladney, E. S.: Atmospheric bromine in the Arctic, J. Geophys. Res., 88C, 6719-6736, 1983.

Berg, W. W., Heidt, L. E., Pollock, W., Sperry, P. D., and Cicerone, R. J.: Brominated organic species in the Arctic atmosphere, Geophys. Res. Lett., 11, 429-432, 1984.

Bergin, M. H., Jaffrezo, J. L., Davidson, C. I., Caldow, R., and Dibb, J.: Fluxes of chemical species to the Greenland ice sheet at Summit by fog and dry deposition, Geochim. Cosmochim. Acta, 58, 3207-3215, 1994.

Bergin, M. H., Jaffrezo, J.-L., Davidson, C. I., Dibb, J. E., Pandis, S. N., Hillamo, R., Maenhaut, W., Kuhns, H. D., and Makela, T.: The contributions of snow, fog, and dry deposition to the summer flux of anions and cations at Summit, Greenland, J. Geophys. Res., 100D, 16 275-16 288, 1995.

Berresheim, H.: Biogenic sulfur emissions from the Subantarctic and Antarctic oceans, J. Geophys. Res., 92D, 13245 $13262,1987$.
Berresheim, H., Huey, J. W., Thorn, R. P., Eisele, F. L., Tanner, D. J., and Jefferson, A.: Measurements of dimethyl sulfide, dimethyl sulfoxide, dimethyl sulfone, and aerosol ions at Palmer Station, Antarctica, J. Geophys. Res., 103D, 16291637, 1998.

Blake, N. J., Blake, D. R., Sive, B. C., Katzenstein, A. S., Meinardi, S., Wingenter, O. W., Atlas, E. L., Flocke, F., Ridley, B. A., and Rowland, F. S.: The seasonal evolution of NMHCs and light alkyl nitrates at middle to high northern latitudes during TOPSE, J. Geophys. Res., 108D, doi: 10.1029/2001JD001467, 2003.

Bloss, W., Lee, J., Heard, D., Salmon, R., Bauguitte, S., Roscoe, H., and Jones, A.: Measurement and interpretation of boundary layer $\mathrm{OH}$ and $\mathrm{HO}_{2}$ in coastal Antarctica, Eos, Trans. AGU (Abstract Supplement), 86/52, A24A-03, 2005.

Bottenheim, J. W., Gallant, A. G., and Brice, K. A.: Measurements of $\mathrm{NO}_{y}$ species and $\mathrm{O}_{3}$ at $82^{\circ} \mathrm{N}$ latitude, Geophys. Res. Lett., 13, 113-116, 1986.

Bottenheim, J. W., Barrie, L. A., Atlas, E., Heidt, L. E., Niki, H., Rasmussen, R. A., and Shepson, P. B.: Depletion of lower tropospheric ozone during Arctic spring: The polar sunrise experiment 1988, J. Geophys. Res., 95D, 18 555-18 568, 1990.

Bottenheim, J. W., Barrie, L. A., and Atlas, E.: The partitioning of nitrogen oxides in the lower Arctic troposphere during spring 1988, J. Atmos. Chem., 17, 15-27, 1993.

Bottenheim, J. W., Boudries, H., Brickell, P. C., and Atlas, E.: Alkenes in the Arctic boundary layer at Alert, Nunavut, Canada, Atmos. Environ., 36, 2585-2594, 2002a.

Bottenheim, J. W., Fuentes, J. D., Tarasick, D. W., and Anlauf, K. G.: Ozone in the Arctic lower troposphere during winter and spring 2000 (ALERT2000), Atmos. Environ., 36, 2535-2544, 2002b.

Boudries, H. and Bottenheim, J. W.: $\mathrm{Cl}$ and $\mathrm{Br}$ atom concentrations during a surface boundary layer ozone depletion event in the Canadian high Arctic, Geophys. Res. Lett., 27, 517-520, 2000.

Boudries, H., Bottenheim, J. W., Guimbaud, C., Grannas, A. M., Shepson, P. B., Houdier, S., Perrier, S., and Dominé, F.: Distribution and trends of oxygenated hydrocarbons in the high Arctic derived from measurements in the atmospheric boundary layer and interstitial snow air during the ALERT2000 field campaign, Atmos. Environ., 36, 25732583, 2002.

Cahill, T. A. and Eldred, R. A.: Elemental composition of Arctic particulate matter, Geophys. Res. Lett., 11, 413-416, 1984.

Carpenter, L. J., Hopkins, J. R., Jones, C. E., Lewis, A. C., Parthipan, R., Wevill, D. J., Poissant, L., Pilote, M., and Constant, P.: Abiotic source of reactive organic halogens in the sub-arctic atmosphere?, Environ. Sci. Technol., 39, 8812-8816, 2005. 
Chance, K.: Analysis of $\mathrm{BrO}$ measurements from the global ozone monitoring experiment, Geophys. Res. Lett., 25, 33353338, 1998.

Cicerone, R. J., Heidt, L. E., and Pollock, W. H.: Measurements of atmospheric methyl bromide and bromoform, J. Geophys. Res., 93D, 3745-3749, 1988.

Clemitshaw, K. C., Ames, D. L., Fleming, Z. L., Salmon, R. A., Bauguitte, S., and Jones, A. E.: Seasonal observations of HONO at Halley, Antarctica during CHABLIS, Geophys. Res. Abstr., 8, 00680 , http://www.cosis.net/abstracts/ EGU06/00680/EGU06-J-00680.pdf, 2006.

Dassau, T. M., Shepson, P. B., Bottenheim, J. W., and Ford, K. M.: Peroxyacetyl nitrate photochemistry and interactions with the Arctic surface, J. Geophys. Res., 109D, D18302, doi:10.1029/2004JD004562, 2004.

Davidson, C. I., Jaffrezo, J.-L., Mosher, B. W., Dibb, J. E., Borys, R. D., Bodhaine, B. A., Rasmussen, R. A., Boutron, C. F., Ducroz, F. M., Cachier, M., Ducret, J., Colin, J.-L., Heidam, N. Z., Kemp, K., , and (1993), R. H.: Chemical constituents in the air and snow at Dye 3, Greenland - II. Analysis of episodes in April 1989, Atmos. Environ., 27A, 2723-2738, 1993a.

Davidson, C. I., Jaffrezo, J.-L., Mosher, B. W., Dibb, J. E., Borys, R. D., Bodhaine, B. A., Rasmussen, R. A., Boutron, C. F., Gorlach, U., Cachier, H., Ducret, J., Colin, J.-L., Heidam, N. Z., Kemp, K., , and Hillamo, R.: Chemical constituents in the air and snow at Dye 3, Greenland - I. Seasonal variations, Atmos. Environ., 27A, 2709-2722, 1993b.

Davis, D., Chen, G., Kasibhatla, P., Jefferson, A., Tanner, D., Eisele, F., Lenschow, D., Neff, W., and Berresheim, H.: DMS oxidation in the Antarctic marine boundary layer: Comparison of model simulations and field observations of DMS, DMSO, DMSO $2, \mathrm{H}_{2} \mathrm{SO}_{4}(\mathrm{~g}), \mathrm{MSA}(\mathrm{g})$, and MSA(p), J. Geophys. Res., 103D, 1657-1678, 1998.

Davis, D., Nowak, J. B., Chen, G., Buhr, M., Arimoto, R., Hogan, A., Eisele, F., Mauldin, L., Tanner, D., Shetter, R., Lefer, B., and McMurry, P.: Unexpected high levels of NO observed at South Pole, Geophys. Res. Lett., 28, 3625-3628, 2001.

Davis, D., Chen, G., Buhr, M., Crawford, J., Lenschow, D., Lefer, B., Shetter, R., Eisele, F., Mauldin, L., and Hogan, A.: South Pole $\mathrm{NO}_{\mathrm{x}}$ chemistry: an assessment of factors controlling variability and absolute levels, Atmos. Environ., $38,5375-5388,2004$.

de Serves, C.: Gas phase formaldehyde and peroxide measurements in the Arctic atmosphere, J. Geophys. Res., 99D, 25 391-25 398, 1994.

Dibb, J. E. and Arsenault, M.: Shouldn't snowpacks be sources of monocarboxylic acids?, Atmos. Environ., 36, 2513-2522, 2002 .

Dibb, J. E., Talbot, R. W., and Bergin, M. H.: Soluble acidic species in air and snow at Summit, Greenland, Geophys. Res. Lett., 21, 1627-1630, 1994.
Dibb, J. E., Talbot, R. W., Shipham, S. I. W. M. C., Winterle, J., McConnell, J., and Bales, R.: Biomass burning signatures in the atmosphere and snow at Summit, Greenland: An event on 5 August 1994, Atmos. Environ., 30, 553-561, 1996.

Dibb, J. E., Talbot, R. W., Munger, J. W., Jacob, D. J., and Fan, S.-M.: Air-snow exchange of $\mathrm{HNO}_{3}$ and $\mathrm{NO}_{y}$ at Summit, Greenland, J. Geophys. Res., 103D, 3475-3486, 1998.

Dibb, J. E., Arsenault, M., Peterson, M. C., and Honrath, R. E.: Fast nitrogen oxide photochemistry in Summit, Greenland snow, Atmos. Environ., 36, 2501-2511, 2002.

Dibb, J. E., Huey, L. G., Slusher, D. L., and Tanner, D. J.: Soluble reactive nitrogen oxides at South Pole during ISCAT 2000, Atmos. Environ., 38, 5399-5409, 2004.

Doskey, P. V. and Gaffney, J. S.: Non-methane hydrocarbons in the Arctic atmosphere at Barrow, Alaska, Geophys. Res. Lett., 19, 381-384, 1992.

Duce, R. A., Winchester, J. W., and van Nahl, T. W.: Iodine, bromine, and chlorine in winter aerosols and snow from Barrow, Alaska, Tellus, 18, 238-248, 1966.

Duce, R. A., Zoller, W. H., and Moyers, J. L.: Particulate and gaseous halogens in the Antarctic atmosphere, J. Geophys. Res., 78, 7802-7811, 1973.

Ebinghaus, R., Kock, H. H., Temme, C., Einax, J. W., Löwe, A. G., Richter, A., Burrows, J. P., and Schroeder, W. H.: Antarctic springtime depletion of atmospheric mercury, Environ. Sci. Technol., 36, 1238-1244, 2002.

Evans, M. J., Jacob, D. J., Atlas, E., Cantrell, C. A., Eisele, F., Flocke, F., Fried, A., Mauldin, R. L., Ridley, B. A., Wert, B., Talbot, R., Blake, D., Heikes, B., Snow, J., Walega, J., Weinheimer, A. J., and Dibb, J.: Coupled evolution of $\mathrm{BrO}_{\mathrm{x}}{ }^{-}$ $\mathrm{ClO}_{\mathrm{x}}-\mathrm{HO}_{\mathrm{x}}-\mathrm{NO}_{\mathrm{x}}$ chemistry during bromine-catalyzed ozone depletion events in the Arctic boundary layer, J. Geophys. Res., 108D, 8368, doi:10.1029/2002JD002732, 2003.

Ferek, R. J., Hobbs, P. V., Radke, L. F., Herring, J. A., Sturges, W. T., and Cota, G. F.: Dimethyl sulfide in the Arctic atmosphere, J. Geophys. Res., 100D, 26 093-26 104, 1995.

Ferrari, C. P., Dommergue, A., Boutron, C. F., Skov, H., Goodsite, M., and B.Jensen: Nighttime production of elemental gaseous mercury in interstitial air of snow at Station Nord, Greenland, Atmos. Environ., 38, 2727-2735, 2004.

Fischer, R., Weller, R., Jacobi, H.-W., and Ballschmiter, K.: Levels and pattern of volatile organic nitrates and halocarbons in the air at Neumayer Station $\left(70^{\circ} \mathrm{S}\right)$, Antarctic, Chemosphere, 48, 981-992, 2002.

Ford, K., Campbell, B., Shepson, P. B., Bertman, S. B., Honrath, R. E., Peterson, M. C., and Dibb, J. E.: Studies of peroxyacetyl nitrate (PAN) and its interaction with the snowpack at Summit, Greenland, J. Geophys. Res., 107D, doi: 10.1029/2001JD000547, 2002.

Foster, K. L., Plastridge, R. A., Bottenheim, J. W., Shepson, P. B., Finlayson-Pitts, B. J., and Spicer, C. W.: The role of $\mathrm{Br}_{2}$ and $\mathrm{BrCl}$ in surface ozone destruction at polar sunrise, Science, 291, 471-474, 2001. 
Frey, M. M., Stewart, R. W., McConnell, J. R., and Bales, R. C.: Atmospheric hydroperoxides in West Antarctica: Links to stratospheric ozone and atmospheric oxidation capacity, J. Geophys. Res., 110D, D23301, doi:10.1029/ 2005JD006110, 2005.

Frieß, U., Wagner, T., Pundt, I., Pfeilsticker, K., and Platt, U.: Spectroscopic measurements of tropospheric iodine oxide at Neumayer station, Antarctica, Geophys. Res. Lett., 28, 1941-1944, 2001.

Frieß, U., Hollwedel, J., König-Langlo, G., Wagner, T., and Platt, U.: Dynamics and chemistry of tropospheric bromine explosion events in the Antarctic coastal region, J. Geophys. Res., 109D, doi:10.1029/2003JD004133, 2004.

Fuhrer, K., Hutterli, M. A., and McConnell, J. R.: Overview of recent field experiments for the study of the air-snow transfer of $\mathrm{H}_{2} \mathrm{O}_{2}$ and $\mathrm{HCHO}$, in: Chemical Exchange Between the Atmosphere and Polar Snow, NATO ASI Series, Vol. I43, edited by Wolff, E. W. and Bales, R. C., pp. 307-318, Springer Verlag, Berlin, 1996.

Galaktionov, V. V., Khattatov, V. U., and Rudakov, V. V.: Aircraft observations of ozone in the Arctic troposphere in April 1994, Atmos. Res., 44, 191-198, 1997.

Gauchard, P.-A., Aspmo, K., Temme, C., Steffen, A., Ferrari, C., Berg, T., Ström, J., Kaleschke, L., Dommergue, A., Bahlmann, E., Magand, O., Planchon, F., Ebinghaus, R., Banic, C., Nagorski, S., Baussand, P., and Boutron, C.: Study of the origin of atmospheric mercury depletion events recorded in Ny-Ålesund, Svalbard, spring 2003, Atmos. Environ., 39, 7620-7632, 2005.

Gong, S. L. and Barrie, L. A.: Trends of heavy metal components in the Arctic aerosols and their relationship to the emissions in the Northern Hemisphere, Sci. Total Environ., 342, 175-183, 2005.

Gong, S. L., Walmsley, J. L., Barrie, L. A., and Hopper, J. F.: Mechanisms for surface ozone depletion and recovery during polar sunrise, Atmos. Environ., 31, 969-981, 1997.

Grannas, A. M., Shepson, P. B., Guimbaud, C., Sumner, A. L., Albert, M., Simpson, W., Dominé, F., Boudries, H., Bottenheim, J., Beine, H. J., Honrath, R., and Zhou, X.: A study of photochemical and physical processes affecting carbonyl compounds in the Arctic atmospheric boundary layer, Atmos. Environ., 36, 2733-2742, 2002.

Gruzdev, A. N., Elokhov, A. S., Makarov, O. V., and Mokhov, I. I.: Some recent results of Russian measurements of surface ozone in Antarctica. A meteorological interpretation, Tellus, 45B, 99-105, 1993.

Guimbaud, C., Grannas, A. M., Shepson, P. B., Fuentes, J. D., Boudries, H., Bottenheim, J. W., Dominé, F., Houdier, S., Perrier, S., Biesenthal, T. B., and Splawn, B. G.: Snowpack processing of acetaldehyde and acetone in the Arctic atmospheric boundary layer, Atmos. Environ., 36, 2743-2752, 2002.
Hansen, A. D. A. and Rosen, H.: Vertical distributions of particulate carbon, sulfur, and bromine in the Arctic haze and comparison with ground-level measurements at Barrow, Alaska, Geophys. Res. Lett., 11, 381-384, 1984.

Hara, K., Osada, K., Matsunaga, K., Iwasaka, Y., Shibata, T., and Furuya, K.: Atmospheric inorganic chlorine and bromine species in Arctic boundary layer of the winter/spring, J. Geophys. Res., 107, doi:10.1029/ 2001JD001008, 2002a.

Hara, K., Osada, K., Nishita, C., Yamagata, S., Yamanocuhi, T., Herber, A., Matsunaga, K., Iwasaka, Y., Nagatani, M., and Nakata, H.: Vertical variations of sea-salt modification in the boundary layer of spring Arctic during the ASTAR 2000 campaign, Tellus, 54B, 361-376, $2002 \mathrm{~b}$.

Hausmann, M. and Platt, U.: Spectroscopic measurement of bromine oxide and ozone in the high Arctic during Polar Sunrise Experiment 1992, J. Geophys. Res., 99D, 25399 $25413,1994$.

Heidam, N. Z.: Crustal enrichments in the Arctic aerosol, Atmos. Environ., 19, 2083-2097, 1985.

Heidam, N. Z., Christensen, J., Wahlin, P., and Skov, H.: Arctic atmospheric contaminants in NE Greenland: levels, variations, origins, transport, transformations and trends 19902001, Sci. Total Environ., 331, 5-28, 2004.

Helmig, D., Boulter, J., David, D., Birks, J. W., Cullen, N. J., Steffen, K., Johnson, B. J., and Oltmans, S. J.: Ozone and meteorological boundary-layer conditions at Summit, Greenland, during 3-21 June 2000, Atmos. Environ., 36, 25952608, 2002.

Herring, J. A., Jaffe, D. A., Beine, H. J., Madronich, S., and Blake, D. R.: High latitude springtime photochemistry part II: Sensitivity studies of ozone production, J. Atmos. Chem., 27, 155-178, 1997.

Hoff, R. M., Leaitch, W. R., Fellin, P., and Barrie, L. A.: Mass size distribution of chemical constituents of the winter Arctic aerosol, J. Geophys. Res., 88C, 10 947-10 956, 1983.

Hönninger, G.: Halogen Oxide Studies in the Boundary Layer by Multi Axis Differential Optical Absorption Spectroscopy and Active Longpath-DOAS, Ph.D. thesis, Institut für Umweltphysik, Universität Heidelberg, Germany, http://www.ub.uni-heidelberg.de/archiv/1940, 2002.

Hönninger, G. and Platt, U.: Observations of $\mathrm{BrO}$ and its vertical distribution during surface ozone depletion at Alert, Atmos. Environ., 36, 2481-2489, 2002.

Hönninger, G., Leser, H., Sebastián, O., and Platt, U.: Ground-based measurements of halogen oxides at the Hudson Bay by longpath DOAS and passive MAX-DOAS, Geophys. Res. Lett., 31, doi:10.1029/2003GL018982, 2004.

Honrath, R. E., Peterson, M. C., Guo, S., Dibb, J. E., Shepson, P. B., and Campbell, B.: Evidence of $\mathrm{NO}_{\mathrm{x}}$ production within or upon ice particles in the Greenland snowpack, Geophys. Res. Lett., 26, 695-698, 1999. 
Honrath, R. E., Lu, Y., Peterson, M. C., Dibb, J. E., Arsenault, M. A., Cullen, N. J., and Steffen, K.: Vertical fluxes of $\mathrm{NO}_{\mathrm{x}}, \mathrm{HONO}$, and $\mathrm{HNO}_{3}$ above the snowpack at Summit, Greenland, Atmos. Environ., 36, 2629-2640, 2002.

Hopper, J. F., Peters, B., Yokouchi, Y., Niki, H., Jobson, B. T., Shepson, P. B., and Muthuramu, K.: Chemical and meteorological observations at ice camp SWAN during Polar Sunrise Experiment 1992, J. Geophys. Res., 99D, 25 489-25 498, 1994a.

Hopper, J. F., Worthy, D. E. J., Barrie, L. A., and Trivett, N. B. A.: Atmospheric observations of aerosol black carbon, carbon dioxide and methane in the high arctic, Atmos. Environ., 28, 3047-3054, 1994b.

Hopper, J. F., Barrie, L. A., Silis, A., Hart, W., Gallant, A. J., and Dryfhout, H.: Ozone and meteorology during the 1994 Polar Sunrise Experiment, J. Geophys. Res., 103D, 14811492, 1998.

Hov, Ø., Penkett, S. A., Isaksen, I. S. A., and Semb, A.: Organic gases in the Norwegian Arctic, Geophys. Res. Lett., 11, 425-428, 1984.

Hov, Ø., Schmidbauer, N., and Oehme, M.: Light hydrocarbons in the Norwegian Arctic, Atmos. Environ., 23, 2471$2482,1989$.

Huey, L. G., Tanner, D. J., Slusher, D. L., Dibb, J. E., Arimoto, R., Chen, G., Davis, D., Buhr, M. P., Nowak, J. B., Mauldin III, R. L., Eisele, F. L., and Kosciuche, E.: CIMS measurements of $\mathrm{HNO}_{3}$ and $\mathrm{SO}_{2}$ at the South Pole during ISCAT 2000, Atmos. Environ., 38, 5411-5421, 2004.

Hutterli, M. A., Röthlisberger, R., and Bales, R. C.: Atmosphere-to-snow-to-firn transfer studies of $\mathrm{HCHO}$ at Summit, Greenland, Geophys. Res. Lett., 26, 1691-1694, 1999.

Hutterli, M. A., McConnell, J. R., Stewart, R. W., Jacobi, H.W., and Bales, R. C.: Impact of temperature-driven cycling of hydrogen peroxide $\left(\mathrm{H}_{2} \mathrm{O}_{2}\right)$ between air and snow on the planetary boundary layer, J. Geophys. Res., 106D, 15 39515 404, 2001.

Hutterli, M. A., McConnell, J. R., Chen, G., Bales, R. C., Davis, D. D., and Lenschow, D. H.: Formaldehyde and hydrogen peroxide in air, snow and interstitial air at South Pole, Atmos. Environ., 38, 5439-5450, 2004.

Ianniello, A., Beine, H. J., Sparapani, R., Di Bari, F., Allegrini, I., and Fuentes, J.: Denuder measurements of gas and aerosol species above Arctic snow surfaces at Alert 2000, Atmos. Environ., 36, 5299-5309, 2002.

Ianniello, A., Sparapani, R., Allegrini, I., Vazzana, C., Mazziotti Gomez de Teran, C., Montagnoli, M., Fino, A., and Felici, A.: Study of nitrogen containing compounds in the polar troposphere, Ann. Chim. Rome, 93, 69-76, 2003.

Impey, G. A., Shepson, P. B., Hastie, D. R., Barrie, L. A., and Anlauf, K. G.: Measurements of photolyzable chlorine and bromine during the Polar Sunrise Experiment 1995, J. Geophys. Res., 102D, 16 005-16 010, 1997.
Impey, G. A., Mihele, C. M., Anlauf, K. G., Barrie, L. A., Hastie, D. R., and Shepson, P. B.: Measurements of photolyzable halogen compounds and bromine radicals during the Polar Sunrise Experiment 1997, J. Atmos. Chem., 34, 21-37, 1999.

Jacobi, H.-W. and Schrems, O.: Peroxyacetyl nitrate (PAN) distribution over the South Atlantic Ocean, Phys. Chem. Chem. Phys., 1, 5517-5521, 1999.

Jacobi, H.-W., Weller, R., and Schrems, T. B. O.: Latitudinal distribution of peroxyacetyl nitrate (PAN) over the Atlantic Ocean, J. Geophys. Res., 104D, 26 901-26 912, 1999.

Jacobi, H.-W., Weller, R., Jones, A. E., Anderson, P. S., and Schrems, O.: Peroxyacetyl nitrate (PAN) concentrations in the Antarctic troposphere measured during the photochemical experiment at Neumayer (PEAN'99), Atmos. Environ., $34,5235-5247,2000$.

Jacobi, H.-W., Frey, M. M., Hutterli, M. A., Bales, R. C., Schrems, O., Cullen, N. J., Steffen, K., and Koehler, C.: Measurements of hydrogen peroxide and formaldehyde exchange between the atmosphere and surface snow at Summit, Greenland, Atmos. Environ., 36, 2619-2628, 2002.

Jacobi, H.-W., Bales, R. C., Honrath, R. E., Peterson, M. C., Dibb, J. E., Swanson, A. L., and Albert, M. R.: Reactive trace gases measured in the interstitial air of surface snow at Summit, Greenland, Atmos. Environ., 38, 1687-1697, 2004.

Jacobi, H.-W., Kaleschke, L., Richter, A., Rozanov, A., and Burrows, J. P.: Observation of a fast ozone loss in the marginal ice zone of the Arctic Ocean, J. Geophys. Res., 111D, doi:10.1029/2005JD006715, 2006.

Jaeschke, W., Beltz, N., Dierssen, J. P., Haunold, W., Krischke, U., Reinecke, A., Salkowski, T., and v. Trümbach, J.: Measurements on the distribution of trace substances in the Arctic troposphere, Atmos. Res., 44, 199-221, 1997.

Jaffrezo, J. L., Davidson, C. I., Legrand, M., and Dibb, J. E.: Sulfate and MSA in the air and snow on the Greenland ice sheet, J. Geophys. Res., 99D, 1241-1254, 1994.

Jefferson, A., Tanner, D. J., Eisele, F. L., Davis, D. D., Chen, G., Crawford, J., Huey, J. W., Torres, A. L., and Berresheim, H.: $\mathrm{OH}$ photochemistry and methane sulfonic acid formation in the coastal Antarctic boundary layer, J. Geophys. Res., 103D, 1647-1656, 1998.

Jobson, B. T., Niki, H., Yokouchi, Y., Bottenheim, J., Hopper, F., and Leaitch, R.: Measurements of $\mathrm{C}_{2}-\mathrm{C}_{6}$ hydrocarbons during the polar sunrise 92 experiment: Evidence for Cl-atom and Br-atom chemistry, J. Geophys. Res., 99D, $25355-25368,1994$.

Jones, A. E., Weller, R., Minikin, A., Wolff, E. W., Sturges, W. T., McIntyre, H. P., Leonard, S. R., Schrems, O., and Bauguitte, S.: Oxidized nitrogen chemistry and speciation in the Antarctic troposphere, J. Geophys. Res., 104D, 21 355$21366,1999$. 
Jones, A. E., Anderson, P. S., Wolff, E. W., Turner, J., Rankin, A. M., and Colwell, S. R.: A role for newly forming sea ice in springtime polar tropospheric ozone loss? Observational evidence from Halley station, Antarctica, J. Geophys. Res., 111D, doi:10.1029/2005JD006566, 2006.

Jourdain, B. and Legrand, M.: Seasonal variations of atmospheric dimethylsulfide, dimethylsulfoxide, sulfur dioxide, methanesulfonate, and non-sea-salt sulfate aerosols at Dumont dUrville (coastal Antarctica) (December 1998 to July 1999), J. Geophys. Res., 106D, 14 391-14 408, 2001.

Jourdain, B. and Legrand, M.: Year-round records of bulk and size-segregated aerosol composition and $\mathrm{HCl}$ and $\mathrm{HNO}_{3}$ levels in the Dumont d'Urville (coastal Antarctica) atmosphere: Implications for sea-salt aerosol fractionation in the winter and summer, J. Geophys. Res., 107D, doi:10.1029/ 2002JD002471, 2002.

Kawamura, K. and Kasukabe, H.: Source and reaction pathways of dicarboxylic acids, ketoacids and dicarbonyls in Arctic aerosols: One year of observations, Atmos. Environ., 30, 1709-1722, 1996.

Keil, A. D. and Shepson, P. B.: Chlorine and bromine atom ratios in the springtime Arctic troposphere as determined from measurements of halogenated volatile organic compounds, J. Geophys. Res., 111, doi:10.1029/2006JD007119, 2006.

Khalil, M. A. K. and Rasmussen, R. A.: Gaseous tracers of arctic haze, Environ. Sci. Technol., 17, 157-164, 1983.

Kieser, B. N., Bottenheim, J. W., Sideris, T., and Niki, H.: Spring 1989 observations of lower tropospheric chemistry in the Canadian high Arctic, Atmos. Environ., 27A, 2979-2988, 1993.

Kreher, K., Johnston, P. V., Wood, S. W., Nardi, B., and Platt, U.: Ground-based measurements of tropospheric and stratospheric $\mathrm{BrO}$ at Arrival Heights, Antarctica, Geophys. Res. Lett., 24, 3021-3024, 1997.

Lazrus, A. L. and Ferek, R. J.: Acidic sulfate particles in the winter Arctic atmosphere, Geophys. Res. Lett., 11, 417-419, 1984.

Leaitch, W. R., Barrie, L. A., Bottenheim, J. W., Li, S. M., Shepson, P. B., Muthuramu, K., and Yokouchi, Y.: Airborne observations related to ozone depletion at polar sunrise, J. Geophys. Res., 99D, 25 499-25 517, 1994.

Lee, J. D., Bloss, W. J., Heard, D. E., Salmon, R. A., Bauguitte, S., and Jones, A. E.: Measurements of $\mathrm{OH}$ and $\mathrm{HO}_{2}$ in coastal Antarctica by laser-induced fluorescence, http://www. cosis.net/abstracts/EGU06/01483/ EGU06-J-01483.pdf, 2006.

Legrand, M. and Pasteur, E. C.: Methane sulfonic acid to nonsea-salt sulfate ratio in coastal Antarctic aerosol and surface snow, J. Geophys. Res., 103D, 10 991-11 006, 1998.

Legrand, M., Ducroz, F., Wagenbach, D., Mulvaney, R., and Hall, J.: Ammonium in coastal Antarctic aerosol and snow: Role of polar ocean and penguin emissions, J. Geophys. Res., 103D, 11 043-11 056, 1998.
Legrand, M., Sciare, J., Jourdain, B., and Genthon, C.: Subdaily variations of atmospheric dimethylsulfide, dimethylsulfoxide, methanesulfonate, and non-sea-salt sulfate aerosols in the atmospheric boundary layer at Dumont dUrville (coastal Antarctica) during summer, J. Geophys. Res., 106D, 14409 $14422,2001$.

Li, S.-M.: Equilibrium of particle nitrite with gas phase HONO: Tropospheric measurements in the high Arctic during polar sunrise, J. Geophys. Res., 99D, 25 469-25478, 1994.

Li, S.-M. and Winchester, J. W.: Resolution of ionic components of late winter Arctic aerosols, Atmos. Environ., 23, 2387-2399, 1989a.

Li, S.-M. and Winchester, J. W.: Geochemistry of organic and inorganic ions of late winter Arctic aerosols, Atmos. Environ., 23, 2401-2415, 1989b.

Li, S.-M., Barrie, L. A., Talbot, R. W., Harriss, R. C., Davidson, C. I., and Jaffrezo, J.-L.: Seasonal and geographic variations of methanesulfonic acid in the Arctic troposphere, Atmos. Environ., 27A, 3011-3024, 1993.

Li, S.-M., Yokouchi, Y., Barrie, L. A., Muthuramu, K., Shepson, P. B., Bottenheim, J. W., Sturges, W. T., and Landsberger, S.: Organic and inorganic bromine compounds and their composition in the Arctic troposphere during polar sunrise, J. Geophys. Res., 99D, 25 415-25 428, 1994.

Lindberg, S. E., Brooks, S., Lin, C.-J., Scott, K., Meyers, T., Chambers, L., Landis, M., and Stevens, R.: Formation of reactive gaseous mercury in the Arctic: Evidence of oxidation of $\mathrm{Hg}^{0}$ to gas-phase $\mathrm{Hg}-\mathrm{II}$ compounds after Arctic sunrise, Water Air Soil Pollut. Focus, 1, 295-302, 2001.

Lindberg, S. E., Brooks, S., Lin, C.-J., Scott, K. J., Landis, M. S., Stevens, R. K., Goodsite, M., and Richter, A.: Dynamic oxidation of gaseous mercury in the Arctic troposphere at polar sunrise, Environ. Sci. Technol., 36, 1245$1256,2002$.

Lu, J. Y., Schroeder, W. H., Barrie, L. A., Steffen, A., Welch, H. E., Martin, K., Lockhart, L., Hunt, R. V., Boila, G., and Richter, A.: Magnification of atmospheric mercury deposition to polar regions in springtime: the link to tropospheric ozone depletion chemistry, Geophys. Res. Lett., 28, 32193222, 2001.

Maenhaut, W., Cornille, P., Pacyna, J. M., and Vitols, V.: Trace element composition and origin of the atmospheric aerosol in the Norwegian Arctic, Atmos. Environ., 23, 2551$2569,1989$.

Martinez, M., Arnold, T., and Perner, D.: The role of bromine and chlorine chemistry for Arctic ozone depletion events in Ny Ålesund and comparison with model calculations, Ann. Geophys., 17, 941-956, 1999.

Mauldin III, R. L., Cantrell, C. A., Zondlo, M. A., Kosciuch, E., Ridley, B. A., Weber, R., and Eisele, F. E.: Measurements of $\mathrm{OH}, \mathrm{H}_{2} \mathrm{SO}_{4}$, and MSA during Tropospheric Ozone Production About the Spring Equinox (TOPSE), J. Geophys. Res., 108D, doi:10.1029/2002JD002295, 2003. 
Mauldin III, R. L., Kosciuch, E., Henry, B., Eisele, F. L., Shetter, R., Lefer, B., Chen, G., Davis, D., Huey, G., and Tanner, D.: Measurements of $\mathrm{OH}, \mathrm{HO}_{2}+\mathrm{RO}_{2}, \mathrm{H}_{2} \mathrm{SO}_{4}$, and MSA at the South Pole during ISCAT 2000, Atmos. Environ., 38, 5423-5437, 2004.

Mickle, R. E., Bottenheim, J. W., Leaitch, W. R., and Evans, W.: Boundary layer ozone depletion during AGASP-II, Atmos. Environ., 23, 2443-2449, 1989.

Miller, H. L., Weaver, A., Sanders, R. W., Arpag, K., and Solomon, S.: Measurements of arctic sunrise surface ozone depletion events at Kangerlussuaq, Greenland $\left(67^{\circ} \mathrm{N}\right.$, 51 $\left.{ }^{\circ} \mathrm{W}\right)$, Tellus, 49B, 496-509, 1997.

Minikin, A., Legrand, M., Hall, J., Wagenbach, D., Kleefeld, C., Wolff, E., Pasteur, E. C., and Ducroz, F.: Sulfurcontaining species (sulfate and methanesulfonate) in coastal Antarctic aerosol and precipitation, J. Geophys. Res., 103D, 10 975-10 990, 1998.

Morin, S., Hönninger, G., Staebler, R. M., and Bottenheim, J. W.: A high time resolution study of boundary layer ozone chemistry and dynamics over the Arctic Ocean near Alert, Nunavut, Geophys. Res. Lett., 32, doi: 10.1029/2004GL022098, 2005.

Morin, S., Savarino, J., Bekki, S., Gong, S., and Bottenheim, J. W.: Pronounced signature of arctic surface ozone depletion events after polar sunrise on $\Delta^{17} \mathrm{O}$ in atmospheric nitrate, Atmos. Chem. Phys. Discuss., 6, 6255-6297, 2006.

Mosher, B. W., Winkler, P., and Jaffrezo, J.-L.: Seasonal aerosol chemistry at Dye 3, Greenland, Atmos. Environ., 27A, 2761-2772, 1993.

Munger, J. W., Jacob, D. J., Fan, S.-M., Colman, A. S., and Dibb, J. E.: Concentrations and snow-atmosphere fluxes of reactive nitrogen at Summit, Greenland, J. Geophys. Res., 104D, 13 721-13 734, 1999.

Murayama, S., Nakazawa, T., Tanaka, M., Aoki, S., and Kawaguchi, S.: Variations of tropospheric ozone concentration over Syowa station, Antarctica, Tellus, 44B, 262-272, 1992.

Muthuramu, K., Shepson, P. B., Bottenheim, J. W., Jobson, B. T., Niki, H., and Anlauf, K. G.: Relationships between organic nitrates and surface ozone destruction during Polar Sunrise Experiment 1992, J. Geophys. Res., 99D, 25369 $25378,1994$.

Narukawa, M., Kawamura, K., Li, S.-M., and Bottenheim, J. W.: Dicarboxylic acids in the Arctic aerosols and snowpacks collected during ALERT 2000, Atmos. Environ., 36, 2491-2499, 2002.

Ockelmann, G. and Georgii, H.-W.: The distribution of sulfur dioxide over the Norwegian Arctic Ocean during summer, Tellus, 36B, 179-185, 1984.

Oltmans, S. J.: Surface ozone measurements in clean air, J. Geophys. Res., 86C, 1174-1180, 1981.
Oltmans, S. J. and Komhyr, W. D.: Surface ozone distributions and variations from 1973-1984 measurements at the NOAA geophysical monitoring for climatic change baseline observatories, J. Geophys. Res., 91D, 5229-5236, 1986.

Oltmans, S. J., Schnell, R. C., Sheridan, P. J., Peterson, R. E., Li, S.-M., Winchester, J. W., Tans, P. P., Sturges, W. T., Kahl, J. D., and Barrie, L. A.: Seasonal surface ozone and filterable bromine relationship in the high Arctic, Atmos. Environ., 23, 2431-2441, 1989.

Oltmans, S. J., Lefohn, A. S., Harris, J. M., Galbally, I., Scheel, H. E., Bodeker, G., Brunke, E., Claude, H., Tarasick, D., Johnson, B. J., Simmonds, P., Shadwick, D., Anlauf, K., Hayden, K., Schmidlin, F., Fujimoto, T., Akagi, K., Meyer, C., Nichol, S., Davies, J., Redondas, A., and Cuevas, E.: Long-term changes in tropospheric ozone, Atmos. Environ., 40, 3156-3173, 2006.

Oncley, S. P., Buhr, M., Lenschow, D. H., Davis, D., and Semmer, S. R.: Observations of summertime NO fluxes and boundary-layer height at the South Pole during ISCAT 2000 using scalar similarity, Atmos. Environ., 38, 5389-5398, 2004 .

Pacyna, J. M. and Ottar, B.: Transport and chemical composition of the summer aerosol in the Norwegian Arctic, Atmos. Environ., 19, 2109-2120, 1985.

Radke, L. F., Lyons, J. H., Hegg, D. A., and Hobbs, P. V.: Airborne observations of Arctic aerosols. I: Characteristics of Arctic haze, Geophys. Res. Lett., 11, 393-396, 1984.

Rahn, K. A. and McCaffrey, R. J.: Compositional differences between Arctic aerosol and snow, Nature, 280, 479-480, 1979 .

Ramacher, B., Rudolph, J., and Koppmann, R.: Hydrocarbon measurements during tropospheric ozone depletion events: Evidence for halogen atom chemistry, J. Geophys. Res., 104C, 3633-3653, 1999.

Rankin, A. M. and Wolff, E. W.: A year-long record of sizesegregated aerosol composition at Halley, Antarctica, J. Geophys. Res., 108D, 4775, doi:10.1029/2003JD003993, 2003.

Rasmussen, A., Kiilsholm, S., Sørensen, J. H., and Mikkelsen, I. S.: Analysis of tropospheric ozone measurements in Greenland, Tellus, 49B, 510-521, 1997.

Rasmussen, R. A. and Khalil, M. A. K.: Gaseous bromine in the Arctic and Arctic haze, Geophys. Res. Lett., 11, 433436, 1984.

Read, K. A., Salmon, R. A., Lewis, A. C., Jones, A. E., and Bauguitte, S.: $\mathrm{OH}$ and halogen atom influence on the variability of non-methane hydrocarbons in the Antarctic boundary layer, Geophys. Res. Abstr., 8, 04 105, http://www.cosis.net/abstracts/EGU06/ 04105/EGU06-J-04105.pdf, 2006.

Richter, A., Wittrock, F., Eisinger, M., and Burrows, J. P.: GOME observations of tropospheric BrO in northern hemispheric spring and summer 1997, Geophys. Res. Lett., 25, 2683-2686, 1998. 
Richter, A., Wittrock, F., Ladstätter-Weißenmayer, A., and Burrows, J. P.: Gome measurements of stratospheric and tropospheric BrO, Adv. Space Res., 29, 1667-1672, 2002.

Ridley, B. A., Atlas, E. L., Montzka, D. D., Browell, E. V., Cantrell, C. A., Blake, D. R., Blake, N. J., Cinquini, L., Coffey, M. T., Emmons, L. K., Cohen, R. C., DeYoung, R. J., Dibb, J. E., Eisele, F. L., Flocke, F. M., Fried, A., Grahek, F. E., Grant, W. B., Hair, J. W., Hannigan, J. W., Heikes, B. J., Lefer, B. L., Mauldin, R. L., Moody, J. L., Shetter, R. E., Snow, J. A., Talbot, R. W., Thornton, J. A., Walega, J. G., Weinheimer, A. J., Wert, B. P., and Wimmers, A. J.: Ozone depletion events observed in the high latitude surface layer during the TOPSE aircraft program, J. Geophys. Res., 108D, doi:10.1029/2001JD001507, 2003.

Riedel, K., Weller, R., and Schrems, O.: Variability of formaldehyde in the Antarctic troposphere, Phys. Chem. Chem. Phys., 1, 5523-5527, 1999.

Riedel, K., Weller, R., Schrems, O., and König-Langlo, G.: Variability of tropospheric hydroperoxides at a coastal surface site in Antarctica, Atmos. Environ., 34, 5225-5234, 2000 .

Roscoe, H. K. and Roscoe, J.: Polar tropospheric ozone depletion events observed in the International Geophysical Year of 1958, Atmos. Chem. Phys., 6, 3303-3314, 2006.

Rudolph, J., Khedim, A., and Wagenbach, D.: The seasonal variation of light nonmethane hydrocarbons in the Antarctic troposphere, J. Geophys. Res., 94, 13 039-13 044, 1989.

Savarino, J., Kaiser, J., Morin, S., Sigman, D. M., and Thiemens, M. H.: Nitrogen and oxygen isotopic constraints on the origin of atmospheric nitrate in coastal Antarctica, Atmos. Chem. Phys. Discuss., 6, 8817-8870, 2006.

Schall, C. and Heumann, K. G.: GC determination of volatile organoiodine and organobromine compounds in Arctic seawater and air samples, Fresenius J. Anal. Chem., 346, 717$722,1993$.

Schnell, R. C., Liu, S. C., Oltmans, S. J., Stone, R. S., Hofmann, D. J., Dutton, E. G., Deshler, T., Sturges, W. T., Harder, J. W., Sewell, S. D., Trainer, M., and Harris, J. M.: Decrease of summer tropospheric ozone concentrations in Antarctica, Nature, 351, 726-729, 1991.

Schofield, R., Johnston, P. V., Thomas, A., Kreher, K., Connor, B. J., Wood, S., Shooter, D., Chipperfield, M. P., Richter, A., von Glasow, R., and Rodgers, C. D.: Tropospheric and stratospheric BrO columns over Arrival Heights, Antarctica, 2002, J. Geophys. Res., 111D, D22310, doi: 10.1029/2005JD007022, 2006.

Schroeder, W. H., Anlauf, K. G., Barrie, L. A., Lu, J. Y., Steffen, A., Schneeberger, D. R., and Berg, T.: Arctic springtime depletion of mercury, Nature, 394, 331-332, 1998.

Sharma, S., Andrews, E., Barrie, L. A., Ogren, J. A., and Lavoué, D.: Variations and sources of the equivalent black carbon in the high Arctic revealed by long-term observations at Alert and Barrow: 1989-2003, J. Geophys. Res., 111D, doi:10.1029/2005JD006581, 2006.
Shepson, P. B., Sirju, A.-P., Hopper, J. F., Barrie, L. A., Young, V., Niki, H., and Dryfhout, H.: Sources and sinks of carbonyl compounds in the Arctic ocean boundary layer: Polar ice floe experiment, J. Geophys. Res., 101D, 21 081$21089,1996$.

Sigg, A., Staffelbach, T., and Neftel, A.: Gas phase measurements of hydrogen peroxide in Greenland and their meaning for the interpretation of $\mathrm{H}_{2} \mathrm{O}_{2}$ records in ice cores, J. Atmos. Chem., 14, 223-232, 1992.

Simpson, W. R., Carlson, D., Hoenninger, G., Douglas, T. A., Sturm, M., Perovich, D., and Platt, U.: First-year sea-ice contact predicts bromine monoxide $(\mathrm{BrO})$ levels better than potential frost flower contact, Atmos. Chem. Phys. Discuss., 6, $11051-11066,2006$.

Sirois, A. and Barrie, L. A.: Arctic lower tropospheric aerosol trends and composition at Alert, Canada: 1980-1995, J. Geophys. Res., 104, 11 599-11618, 1999.

Skov, H., Christensen, J. H., Goodsite, M. E., Heidam, N. Z., Jensen, B., Wåhlin, P., and Geernaert, G.: Fate of elemental mercury in the Arctic during atmospheric mercury depletion episodes and the load of atmospheric mercury to the Arctic, Environ. Sci. Technol., 38, 2373-2382, 2004.

Skov, H., Wahlin, P., Christensen, J., Heidam, N. Z., and Petersen, D.: Measurements of elements, sulphate and SO2 in Nuuk Greenland, Atmos. Environ., 40, 4775-4781, 2006.

Slusher, D. L., Huey, L. G., Tanner, D. J., Chen, G., Davis, D. D., Buhr, M., Nowak, J. B., Eisele, F. L., Kosciuch, E., Mauldin, R. L., Lefer, B. L., Shetter, R. E., and Dibb, J. E.: Measurements of pernitric acid at the South Pole during ISCAT 2000, Geophys. Res. Lett., 29, doi: 10.1029/2002GL015703, 2002.

Solberg, S., Dye, C., Schmidbauer, N., Herzog, A., and Gehrig, R.: Carbonyls and nonmethane hydrocarbons at rural European sites from the mediterranean to the arctic, J. Atmos. Chem., 25, 33-66, 1996a.

Solberg, S., Schmidbauer, N., Semb, A., Stordal, F., and Hov, $\varnothing .:$ Boundary layer ozone depletion as seen in the Norwegian Arctic in spring, J. Atmos. Chem., 23, 301-332, 1996b.

Solberg, S., Krognes, T., Stordal, F., Hov, Ø., Beine, H. J., Jaffe, D. A., Clemitshaw, K., and Penkett, S. A.: Reactive nitrogen compounds at Spitsbergen in the Norwegian Arctic, J. Atmos. Chem., 28, 209-225, 1997a.

Solberg, S., Stordal, F., and Hov, Ø.: Tropospheric ozone at high latitudes in clean and polluted air masses, a climatological study, J. Atmos. Chem., 28, 111-123, 1997b.

Sommar, J., Wängberg, I., Berg, T., Gårdfeldt, K., Munthe, J., Richter, A., Urba, A., Wittrock, F., and Schroeder, W. H.: Circumpolar transport and air-surface exchange of atmospheric mercury at $\mathrm{Ny}$-Ålesund $\left(79^{\circ} \mathrm{N}\right)$, Svalbard, spring 2002, Atmos. Chem. Phys., 7, 151-166, 2007.

Spicer, C. W., Plastridge, R. A., Foster, K. L., Finlayson-Pitts, B. J., Bottenheim, J. W., Grannas, A. M., and Shepson, P. B.: Molecular halogens before and during ozone depletion events in the Arctic at polar sunrise: concentrations and sources, Atmos. Environ., 36, 2721-2731, 2002. 
Sprovieri, F., Pirrone, N., Landis, M. S., and Stevens, R. K.: Atmospheric mercury behavior at different altitudes at $\mathrm{Ny}$ Ålesund during Spring 2003, Atmos. Environ., 39, 76467656, 2005a.

Sprovieri, F., Pirrone, N., Landis, M. S., and Stevens, R. K.: Oxidation of gaseous elemental mercury to gaseous divalent mercury during 2003 polar sunrise at Ny-Ålesund, Environ. Sci. Technol., 39, 9156-9165, 2005b.

Steffen, A., Schroeder, W., Bottenheim, J., Narayan, J., and Fuentes, J. D.: Atmospheric mercury concentrations: measurements and profiles near snow and ice surfaces in the Canadian Arctic during Alert 2000, Atmos. Environ., 36, 2653-2661, 2002.

Steffen, A., Schroeder, W. H., Edwards, G., and Banic, C.: Mercury throughout polar sunrise 2002, J. Phys. IV France, 107, 1267-1270, 2003.

Steffen, A., Schroeder, W., Macdonald, R., Poissant, L., and Konoplev, A.: Mercury in the Arctic atmosphere: An analysis of eight years of measurements of GEM at Alert (Canada) and a comparison with observations at Amderma (Russia) and Kuujjuarapik (Canada), Sci. Total Environ., 342, 185198, 2005.

Sturges, W. T. and Barrie, L. A.: Chlorine, bromine and iodine in Arctic aerosols, Atmos. Environ., 22, 1179-1194, 1988.

Sturges, W. T. and Shaw, G. E.: Halogens in aerosols in central Alaska, Atmos. Environ., 27A, 2969-2977, 1993.

Sturges, W. T., Hopper, J. F., Barrie, L. A., and Schnell, R. C.: Stable lead isotope ratios in Alaskan Arctic aerosols, Atmos. Environ., 27A, 2865-2871, 1993a.

Sturges, W. T., Schnell, R. C., Dutton, G. S., Garcia, S. R., and Lind, J. A.: Spring measurements of tropospheric bromine at Barrow, Alaska, Geophys. Res. Lett., 20, 201-204, 1993b.

Sturges, W. T., Schnell, R. C., Landsberger, S., Oltmans, S. J., Harris, J. M., and Li, S. M.: Chemical and meteorological influences on surface ozone destruction at Barrow, Alaska, during spring 1989, Atmos. Environ., 27A, 2851-2863, 1993c.

Sturges, W. T., Sullivan, C. W., Schnell, R. C., Heidt, L. E., and Pollock, W. H.: Bromoalkane production by Antarctic ice algae, Tellus, 45B, 120-126, 1993d.

Sumner, A. L. and Shepson, P. B.: Snowpack production of formaldehyde and its effect on the Arctic troposphere, Nature, 398, 230-233, 1999.

Sumner, A. L., Shepson, P. B., Grannas, A. M., Bottenheim, J. W., Anlauf, K. G., Worthy, D., Schroeder, W. H., Steffen, A., Dominé, F., Perrier, S., and Houdier, S.: Atmospheric chemistry of formaldehyde in the Arctic troposphere at Polar Sunrise, and the influence of the snowpack, Atmos. Environ., 36, 2553-2562, 2002.

Swanson, A. L., Blake, N. J., Atlas, E., Flocke, F., Blake, D. R., and Rowland, F. S.: Seasonal variations of $\mathrm{C}_{2}-\mathrm{C}_{4}$ nonmethane hydrocarbons and $\mathrm{C}_{1}-\mathrm{C}_{4}$ alkyl nitrates at the Summit research station in Greenland, J. Geophys. Res., 108D, doi:10.1029/2001JD001445, 2003.
Swanson, A. L., Davis, D. D., Arimoto, R., Roberts, P., Atlas, E. L., Flocke, F., Meinardi, S., Rowland, F. S., and Blake, D. R.: Organic trace gases of oceanic origin observed at South Pole during ISCAT 2000, Atmos. Environ., 38, 54635472, 2004.

Taalas, P., Kyrö, E., Supperi, A., Tafuri, V., and Ginzburg, M.: Vertical distribution of tropospheric ozone in Antarctica and in the European Arctic, Tellus, 45B, 106-119, 1993.

Tackett, P. J., Cavender, A. E., Keil, A. D., Shepson, P. B., Bottenheim, J. W., Morin, S., Deary, J., Steffen, A., and Doerge, C.: A study of the vertical scale of halogen chemistry in the Arctic troposphere during polar sunrise at Barrow, Alaska, J. Geophys. Res., (in press), 2006.

Tarasick, D. W. and Bottenheim, J. W.: Surface ozone depletion episodes in the Arctic and Antarctic from historical ozonesonde records, Atmos. Chem. Phys., 2, 197-205, 2002.

Teinilä, K., Hillamo, R., Kerminen, V.-M., and Beine, H. J.: Aerosol chemistry during the NICE dark and light campaigns, Atmos. Environ., 37, 563-575, 2003.

Trivett, N. B. A., Worthy, D. E. J., and Brice, K. A.: Surface measurements of carbon dioxide and methane at Alert during an Arctic haze event in April, 1986, J. Atmos. Chem., 9, 383-397, 1989.

Tuckermann, M., Ackermann, R., Gölz, C., Lorenzen-Schmidt, H., Senne, T., Stutz, J., Trost, B., Unold, W., and Platt, U.: DOAS-observation of halogen radical-catalysed Arctic boundary layer ozone destruction during the ARCTOC campaign 1995 and 1996 in Ny-Ålesund, Spitzbergen, Tellus, 49B, 533-555, 1997.

Wagenbach, D., Ducroz, F., Mulvaney, R., Keck, L., Minikin, A., Legrand, M., Hall, J. S., and Wolff, E. W.: Sea-salt aerosol in coastal Antarctic regions, J. Geophys. Res., 103D, 10961-10 974, 1998a.

Wagenbach, D., Legrand, M., Fischer, H., Pichlmayer, F., and Wolff, E. W.: Atmospheric near-surface nitrate at coastal Antarctic sites, J. Geophys. Res., 103D, 1100711020,1998 b.

Wagner, T. and Platt, U.: Satellite mapping of enhanced BrO concentrations in the troposphere, Nature, 395, 486-490, 1998.

Wang, Y., Choi, Y., Zeng, T., Davis, D., Buhr, M., Huey, L. G., and Neff, W.: Assessing the photochemical impact of snow $\mathrm{NO}_{\mathrm{x}}$ emissions over Antarctica during ANTCI 2003, Atmos. Environ., (submitted), 2006.

Weller, R., Minikin, A., König-Langlo, G., Schrems, O., Jones, A. E., Wolff, E. W., and Anderson, P. S.: Investigating possible causes of the observed diurnal variability in Antarctic $\mathrm{NO}_{\mathrm{y}}$, Geophys. Res. Lett., 26, 2853-2856, 1999.

Weller, R., Jones, A. E., Wille, A., Jacobi, H.-W., McIntyre, H. P., Sturges, W. T., Huke, M., and Wagenbach, D.: Seasonality of reactive nitrogen oxides $\left(\mathrm{NO}_{\mathrm{y}}\right)$ at $\mathrm{Neu}-$ mayer Station, Antarctica, J. Geophys. Res., 107D, doi: 10.1029/2002JD002495, 2002. 
Wessel, S., Aoki, S., Winkler, P., Weller, R., Herber, A., Gernandt, H., and Schrems, O.: Tropospheric ozone depletion in polar regions: A comparison of observations in the Arctic and Antarctic, Tellus, 50B, 34-50, 1998.

Winchester, J. W., Schnell, R. C., Fan, S., Li, S., Bodhaine, B. A., Naegele, P. S., Hansen, A. D. A., and Rosen, H.: Particulate sulfur and chlorine in Arctic aerosols, spring 1983, Atmos. Environ., 19, 2167-2173, 1985.

Wingenter, O. W., Sive, B. C., Blake, D. R., Rowland, F. S., and Ridley, B. A.: Unexplained enhancements of $\mathrm{CH}_{3} \mathrm{Br}$ in the Arctic and sub-Arctic lower troposphere during TOPSE spring 2000, Geophys. Res. Lett., 30, doi: 10.1029/2003GL018159, 2003.

Wittrock, F., Müller, R., Richter, A., Bovensmann, H., and Burrows, J. P.: Measurements of iodine monoxide (IO) above Spitsbergen, Geophys. Res. Lett., 27, 1471-1474, 2000.

Wolff, E. W. and Cachier, H.: Concentrations and seasonal cycle of black carbon in aerosol at a coastal Antarctic station, J. Geophys. Res., 103D, 11 033-11 042, 1998.

Worthy, D. E. J., Trivett, N. B. A., Hopper, J. F., and Bottenheim, J. W.: Analysis of long-range transport events at
Alert, Northwest Territories, during the Polar Sunrise Experiment, J. Geophys. Res., 99D, 25 329-25 344, 1994.

Yang, J., Honrath, R. E., Peterson, M. C., Dibb, J. E., Sumner, A. L., Shepson, P. B., Frey, M., Jacobi, H.-W., Swanson, A., and Blake, N.: Impacts of snowpack emissions on deduced levels of $\mathrm{OH}$ and peroxy radicals at Summit, Greenland, Atmos. Environ., 36, 2523-2534, 2002.

Yokouchi, Y., Akimoto, H., Barrie, L. A., Bottenheim, J. W., Anlauf, K. G., and Jobson, B. T.: Serial gas chromatographic/mass spectrometric measurements of some volatile organic compounds in the Arctic atmosphere during the 1992 Polar Sunrise Experiment, J. Geophys. Res., 99D, 25379 $25389,1994$.

Zhou, X., Beine, H. J., Honrath, R. E., Fuentes, J. D., Simpson, W., Shepson, P. B., and Bottenheim, J. W.: Snowpack photochemical production of HONO: a major source of $\mathrm{OH}$ in the Arctic boundary layer in springtime, Geophys. Res. Lett., 28, 4087-4090, 2001.

Zoller, W. H., Gladney, E. S., and Duce, R. A.: Atmospheric concentrations and sources of trace metals at the South Pole, Science, 183, 198-200, 1974. 